\title{
Incomplete contracts and the core
}

Citation for published version (APA):

Predtetchinski, A. (2004). Incomplete contracts and the core. [Doctoral Thesis, Maastricht University]. Datawyse / Universitaire Pers Maastricht. https://doi.org/10.26481/dis.20041112ap

Document status and date:

Published: 01/01/2004

DOI:

10.26481/dis.20041112ap

Document Version:

Publisher's PDF, also known as Version of record

\section{Please check the document version of this publication:}

- A submitted manuscript is the version of the article upon submission and before peer-review. There can be important differences between the submitted version and the official published version of record.

People interested in the research are advised to contact the author for the final version of the publication, or visit the DOI to the publisher's website.

- The final author version and the galley proof are versions of the publication after peer review.

- The final published version features the final layout of the paper including the volume, issue and page numbers.

Link to publication

\footnotetext{
General rights rights.

- You may freely distribute the URL identifying the publication in the public portal. please follow below link for the End User Agreement:

www.umlib.nl/taverne-license

Take down policy

If you believe that this document breaches copyright please contact us at:

repository@maastrichtuniversity.nl

providing details and we will investigate your claim.
}

Copyright and moral rights for the publications made accessible in the public portal are retained by the authors and/or other copyright owners and it is a condition of accessing publications that users recognise and abide by the legal requirements associated with these

- Users may download and print one copy of any publication from the public portal for the purpose of private study or research.

- You may not further distribute the material or use it for any profit-making activity or commercial gain

If the publication is distributed under the terms of Article $25 \mathrm{fa}$ of the Dutch Copyright Act, indicated by the "Taverne" license above, 
Incomplete Contracts and the Core 
This book was typeset by the author using ITEX.

(C) Arkadi Predtetchinski, 2004

All rights reserved. No part of this publication may be reproduced, stored in a retrieval system, or transmitted, in any form, or by any means, electronic, mechanical, photocopying, recording or otherwise, without the prior permission in writing, from the author.

Published by Universitaire Pers Maastricht

ISBN 9052784310

Printed in the Netherlands by Datawyse 


\title{
Incomplete Contracts and the Core
}

\author{
PROEFSCHRIFT
}

ter verkrijging wan de graad van doctor an

de Universiteit Maastricht,

op gezag van de Rector Magnificus,

Prof. mr. G.P.M.F. Mols,

volgens het besluit van het College van Decanen

in het openbaar te verdedigen op

vrijdag 12 november 2004 om 10:00 uur

door

Arkadi Predtetchinshi

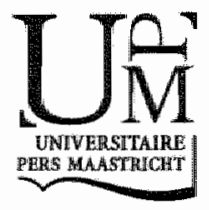


Promotores:

Prof. dr. P.J.J. Herings

Prof. dr. H.J.M. Peters

Beoordelingscommissie:

Prof. dr. G. Kirchsteiger (voorzitter)

Prof. dr. S. Kokovin (Novosibirsk State University, Russia)

Dr. A. Perea 


\section{Acknowledgements}

This study is the result of research carried over a period of four years at the Faculty of Economics and Business Administration of the University of Maastricht. All financial support from the Faculty is gratefully acknowledged.

My first thanks go to my supervisors Jean-Jacques Herings and Hans Peters. This work woulc have never been accomplished without their guidance and relentless support. I am also indebted to Andrés Perea for many invaluable discussions. Special thanks to Georg Kirchsteiger for chairing the promotion committee and Serguei Kokovin for taking part in this committee. Furthermore, I would like to thank all my colleagues at the Faculty for a friendly and comfortable working atmosphere.

For prowiding me with support and consolation when I most needed them and for sharing in my successes, I would like to thank my mother.

Arkadj Predtetchinski

Maastricht:

September 3, 2004 



\section{Contents}

1 Introduction

I Payoff-dependent Balancedness and the Core

2 A Necessary and Sufficient Condition for the Non-emptiness of the Core of a Non-transferable Utility Grame

2.1 Introduction . . . . . . . . . . . . . . . . . . . . . . . 9

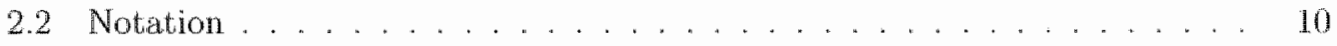

$2.3 \pi$-Balancedness and $(\pi,<)$-balancedness . . . . . . . . . . . 11

$24 \Pi$-Balancedness . . . . . . . . . . . . . . . . . . . 12

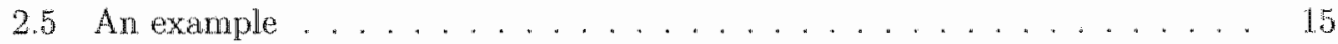

2.6 The $\Psi$-Balanced core . . . . . . . . . . . . . . . . . 16

3 The Fuzzy Core and the $\Psi$-Balanced Core $\quad 21$

3.1 Introduction ......................... 21

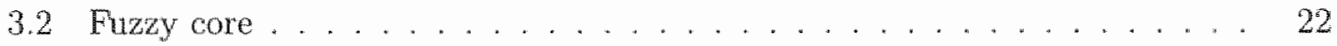

3.3 The non emptiness of the $6-$ fuzzy core . . . . . . . . . . . . . 23

II Sequential Core Concepts 29

4 The Strong Sequential Core in a Two-period Economy 31

4.1 Introduction . . . . . . . . . . . . . . . . . . . 31

4.2 The model and preliminaries . . . . . . . . . . . . . . . . . . . . . . . . . . . . . . . . . .

4.3 The strong sequential core . . . . . . . . . . . . . . . . . . . . . . . . . . . . . . . . . . . .

4.4 Finance economies . . . . . . . . . . . . . . . . . . . . . . . . . . . . . . . . . . . . . . . . . . . . . . .

4.5 The multiple commodities case ... . . . . . . . . . . . . . . . 40

4.6 The strong* sequential core ... . . . . . . . . . . . . . . . 42

5 The Weak Sequential Core in a Two-period Economy' 47

5.1 Introduction . . . . . . . . . . . . . . . . . . . 47

5.2 Constrained feasible allocations and ex post subecononies . . . . . . 48

5.3 Three concepts of the sequential core . . . . . . . . . . . . . . . . 49 
5.4 Some special cases . . . . . . . . . . . . . . . . . . . . 51

5.5 An NTU-characterization of the weak sequential cort . . . . . . . . 53

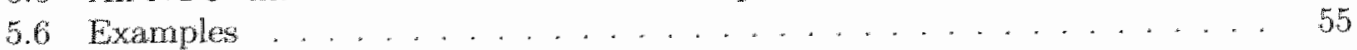

6 The Strong Sequential Come in a Stationary Exchange Economy 67

6.1 Introduction . . . . . . . . . . . . . . . . . . 67

6.2 Stationary exchange economies . . . . . . . . . . . . . 68

6.3 A characterization of the strong sequential core . . . . . . . . . . . . 71

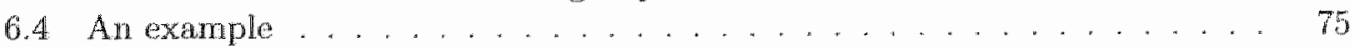

6.5 A convergence theorem . . . . . . . . . . . . . . . 77

6.6 A non-emptincss result . . . . . . . . . . . . . . . . . . . . . . . . . . . . . . . . . . . . . . . . .

6.7 Concluding remarks . . . . . . . . . . . . . . . . 81

7 The Strong Sequential Core in a Stationary Cooperative Game 83

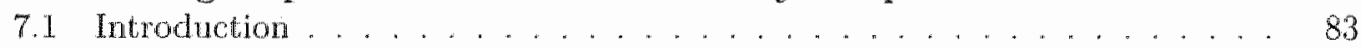

7.2 Stationary cooperative games . . . . . . . . . . . . . . 84

7.3 A characterization of the strong sequential core . . . . . . . . . . . 86

7.4 Additive $\pi$-balancedness and strong additive

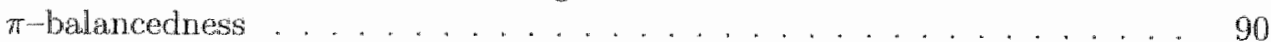

7.5 Existence results . . . . . . . . . . . . . . . . . . . 92

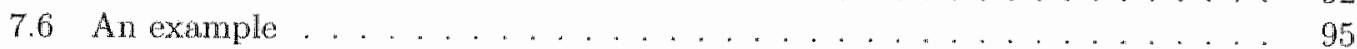

$\begin{array}{lr}\text { Bibliography } & 99\end{array}$

$\begin{array}{lr}\text { Index } & 102\end{array}$

$\begin{array}{lr}\text { Summary } & 103\end{array}$

$\begin{array}{lr}\text { Curriculum Vitae } & 107\end{array}$ 


\section{Chapter 1}

\section{Introduction}

Cooperative game theory is a discipline that studies collective decision making in a group of individuals. The distinctive feature of the cooperative theory of games is the assumption that the players are able to communicate and coordinate their strategies. While each player is considered selfish, i.e. acting so as to maximize his own well-being, the players in a cooperative game may choose to act coherently, whenever this allows them to jointly increase their welfare.

Arguably the core is the most important solution concept in cooperative game theory. In my understanding, the importance of the core in the classical theory of games can be explained by its possessing the following features. Generality: the core of a game or of an economy consists of those outcomes that are robust against all coalitional cleviations. While the precise definition of a coalitional deviation depends on the specifics of the situation in question, the underlying principle is very general. Coalitional stability can be defined for many game-theoretic and economic situations such as exchange and production economies, games in characteristic form, and games in normal form. Intuitive appeal: coalitional stability can be seen as a necessary condition for an allocation to be implemented by the cooperating players. It is difficult to argue that an allocation which can be improved upon by some group of players will be chosen as an outcome in a cooperative game. Tractabilty: the definition of the classical core is simple. Many properties of this solution concept, most. notably, its non-emptiness, have been studied extensively.

The present monograph is entirely devoted to the concept of the core. Two principle topics covered in the book are: (I) Payoff-dependent Balancedness and the Core, and (II) Sequential Core Concepts. The book is correspondingly divided in two parts, with balancedness and related issues addressed in Part I (Chapters 2 and 3), and the sequential core concepts delegated to Part II (Chapters 4 through 7). In Part I we work with the notions of a game without side payments, balancedness and fuzzy core. Most of these concepts have been studied extensively since the 1960s in the celebrated works of Aumann, Shapley, Scarf, and others. In contrast, Part II is an excursion to the field of dynamic. cooperation which has only tentatively been addressed in the literature. Throughout this monograph we try to stay in line with the requirements of generality, intuitive appeal, and tractability. When addressing balancedness in cooperative games, we seek to obtain the 
most general condition for the non-emptiness of the core; when discussing concepts of t sequential core, we argue intuitively as well as formally, and we always try to make or anclysis transparent and avoid any unnecessary sophistication.

Part I of the monograph contributes to the literature on balancedness in cooperatix games without side payments. The class of games without side payments, or games wit non-transferable utility, was introduced in Aumann and Peleg [3] and in Aumann [2] $\varepsilon$ a generalization of games in characteristic function form defined by von Neumann an Morgenstern [34]. (Since then the games considered by von Neumann and Morgenster are known as games with transferable utility.) A game with non-transferable utility (NTU game) is among the most general and abstract settings to analyze the concept of the cor It consists of the set of players and a function that assigns to each coalition of players set of utility allocations. This function provides an exhaustive description of what eacl coalition can achieve in terms of the utility payoffs, when acting independently of th outside players. The core of an NTU-game is defined as the set of allocations that ar feasible for the grand coalition and robust against all coalitional deviations.

It is well-known that a transferable utility game has a non-empty core if and only if it it balanced. In the class of non-transferable utility games balancedness in the sense of Scar [30] or the more general $\pi$-balancedness due to Billera [4] are sufficient but not necessary conditions for the core to be non-empty. In Chapter 2 of this monograph we propose a new balancedness condition for a general NTU-game. Our balancedness condition is a natura. generalization of the balancedness in the sense of Billera [4], and is not only a sufficient. but also a necessary condition for the non-emptiness of the core. We are thus able to give a precise characterization of the class of NTU-games with a non-empty core. In Chapter 3 we employ the techniques developed in Chapter 2 to give a new proof of the non-emptiness of the fuzzy cote in a pure exchange economy.

Part II of this monograph contributes to the field of dynamic cooperative game theory. Unlike the thoroughly developed theory of dynamic non-cooperative games, the theory of dynamic cooperation is yet hardly distinguishable as a separate field. The literature on dymamic cooperative games is scarce and fragmented, often dominated by ad hoc assumptions, and lacks a common understanding of the principles of cooperation over time. We focus on specific question: how to extend the concept of the core to a dynamic setup? While cxamining several possibilities to do so, we illustrate that the development of a consistent theory of dynamic cooperative games presents a number of challenges, and has an enormous researcl potential

When one focuses on cooperative solution concepts, the distinction between static and dynamic environments lies in the enforceability of agreements. In a static economy, all agreements can be regarded as enforceable. Indeed, it makes no difference whether we consider a player as merely agreeing to undertake a certain action or actually carrying out that action, because nothing happens in between. In this respect dynamic economies are drastically different from static economies. In a dynamic economy, a player can deviate from an agreement that has been only partially carried out. Therefore the distinction between those agreements that are fully enforceable and those that are not becomes crucial
in a dymamic setup. 
As was illustrated in Kranich, Perea, and Peters [20], the incentives to deviate from earlier agreements can arise even in the most simple deterministic environments. In the present monograph, however, we consider economies and games with uncertainty that resolves gradually over time. In this context, the incentives to deviate are caused by the increase in the information possessed by the players.

The classical core, being a general and flexible solution concept, can be applied to a dynamic economy with uncertainty. In this context, the classical core selects those allocations that cannot be improved upon by any coalition at the beginning of time. For this reason, we call the classical core in a dynamic economy with uncertainty the ex ante core. The ex ante core renders all plans regarding trade in the future binding. To put it equivalently, whenever all possible contracts are binding, the classical core is an appropriate solution concept. With only a limited number of agreements (or none at: all) being enforceable, some of the allocations in the classical core may not be implementable, for coalitions may have incentives to deviate from such agreements in the course of the game.

We illustrate this point by an example. Consider an econony with two players called $a$ and $b$, and two periods of time (period $t=0$ and period $t=1$ ). In period $t=1$ the economy can be in one of two equally probable states. There is one commodity in each state. In state $s=1$ player $a$ owns 1 unit of commodity, while player $b$ 's endowment is 0 . In state $s=2$ the endowment of player $a$ is 0 , while that of player $b$ is 1 . Assume that both players are risk-averse von Neumann-Morgenstern expected utility maximizers.

In such an economy the set of the ex ante Pareto-efficient allocations coincides with the diagonal of the Edgeworth box. An allocation that assigns each agent half a unit of the commodity in each state is Pareto-efficient and individually rational for both players. It is in the ex ante core: no coalition has an incentive to deviate in period $t=0$. According to the above agreement, however, if state $s=1$ occurs in period $t=1$, then player a should deliver player $b$ a positive amount of the commodity. Thus player $a$ is in the pasition to improve upon the proposed allocation ex post, provided that state $s=1$ occurs. If an agreement to share the endowments equally in each state cannot be legally enforced, it is unlikely to be the outcome of cooperation.

These considerations motivate us to consider those solution concepts that impose conditions of self-enforcement at any time period and state of nature that can be reached in the course of the game. We refer to any such solution concept as a secuential core. As was pointed out earlier, the literature on cooperative solution concepts for dynamic environments is scarce. Various definitions of sequential core were proposed in Gale [11], in Kranich, Perea, and Peters [20], in Koutsougeras [17], in Koutsougeras and Shafer [1.9], in Koutsougeras [18] and in Marakulin [21]. This small amount of work does not allow us to speak of any common approach to the definition of a sequential core. Below we discuss several definitions, with the emphasis on the strong sequential core and the weak secuential core.

In my understanding, the major difficulty that hinders the development, of a universal approach to modelling cooperation in a dynamic setup is the one of specifying the players' expectations. In most dynamic models, the utility of a player at a given period of time depends on his consumption in the future, as well as on the consumption at that moment. 
of time. Therefore, in order to evaluate the profitability of a deviation, the players in a deviating coalition should be able to form some expectations as to the play of the game following their deviation. To decide whether the deviation pays off or not, the players should anticipate their consumption in the future. Any concept of the sequential corte must therefore specify the players' expectations as to what happens in the game following a coalitional deviation.

The concept of the strong sequential core was introduced by Gale [11] in the framework of a monetary multi-period economy with uncertainty. Kranich, Perea, and Peters [20] studied the concept in deterministic setting where the players face a finte stquence of transferable utility games. To define the strong sequential core, one identifies the appropriate sub-economies (or sul-games) of a given dynamic economy (or a game). The net trade in commodities (or the allocations of utilities, depending on the context) is in the strong sequential core, if it belongs to the classical core of every sub economy. In the above axample, there are three sub-economies: the sub econorny corresponding to state $s=1$ of period $t=1$, the sub-economy corresponding to state $s=2$ of period $t=1$, and the ax ante sub-economy, corresponding to period $t=0$. The cores of the two sub-economies in period $t=1$ both consist of one point only: the initial allocation. Since the initial allocation is not in the ex ante core, the strong sequential core is empty.

A closer examination of the above definition reveals the following behavioral assumptions underlying the concept of the strong sequential core. We assume that coalitions deviate forever: once the coalition has deviated, it cannot join the rest of society later on. At the moment of deviation, the players in the deviating coalition agree to a certain plan that specifies their actions (tracle) for the rest of time. No specific requirements are imposed upon the agreement, apart from the usual feasibility condition. Furthermore, an: agreement within the deviating coalition is seen by the players as binding, for each player expects his partners in the coalition to adhere to such an agreement.

The assumption that the deviating coalition is able to produce binding agreements is indeed not easy to justify: since the grand coalition lacks the capacity to enforce its decisions, one would not in general expect smaller coalitions to possess such a capability. The strong sequential core may therefore be seen as providing a very pessimistic scenario of cooperation, whthe grand coalition being relatively weak, and the deviating coallition. possessing much blocking power. Although vulnerable to criticism, the strong sequential core is nevertheless valuable as a benchmark for other concepts of the sequential core.

The concept of the weak sequential core was first explicitly considered in Kranich, Perea, and Peters $[20]$. This concept drops the assumption that the deviating coalition be able to chforce agreements. It is required instead that the agreement within the coalition be robust against; possible deviations of sub-coalitions. Thus an allocation belongs to the weak sequential core it it is robust aganst all stable (or credible) deviations. It is easy to understand that the strong sequential cone is a subset of the weak sequential core. In the example discussed before, the grand coalition has no credible deviations from the initial allocation: ary other allocation violates the condicion of individual rationality for either player $a$ or player $b$ in one of the states of period $t=1$. Therefore, the initial allocation is in the weak sequential core. It is easy to see that the weak sequential core contains no 
other allocations.

The concepts of the strong sequential core and the weak sequential core render the players' expectations implicitly: expectations are endogenous in the model, and assume the form of agreements achieved within the deviating coalition. Koutsongeras and Shafer [19] and Koutsougeras [18] proposed a family of sequential core concepts where the expectations of the players in the deviating coalition are explicitly and exogenously specified by the model. Two special cases are the so-called two-stage cone, originally proposed in Koutsongeras [17], and the rational expectations core.

In the two-stage core, the deviating coalition is active only at the moment of deviation: while at that moment some trade is carried out, the players in the deviating coalition do not expect to trade in subsequent periods. One possible interpretation is that, being unable to correctly foresee the development of the game, the players incorporate the most pessimistic scenario (no trade) in their expectations. One can demonstrate that, under standard conditions, the weak sequential core is a subset of the two-stage core.

In the rational expectations core, a coalition deviates for at most one period, after which it joins the rest of society. Thus the players who contemplate to deviate at period $t$ expect to always trade in accordance with the proposed allocation, except for period $t$, at which moment they trade only with each other and not with the outside players. There is no logical relation between the rational expectations core and the strong (or weak) sequential core. All equilibria of a two-period economy with real assets are contained both in the two-stage core and in the rational expectations core.

The above discussion shows that there are many ways to specify the players" expectations, and that different specifications may lead to drastically different solution concepts. This multiplicity of possible definitions of the core in a dynamic setting prompts the examination of the formal properties of these definitions.

In Chapters 4 and 5 we consider the strong sequential core and the weak sequential core in the context of two-period economies with real assets. A two period economy with assets has all those ingredients we are interested in: time, uncertainty, and the incentives for the ex post deviations from the ex ante agreements. The class of two-period economies with real assets has been extensively studied in general equilibrium theory, which typically focuses on the implications of market incompleteness. As in general equilibrium theory, we are particularly interested in the implications of incompleteness of the set of available assets for the solution concepts in question. More specifically, we ask the following three questions:

(1) How does the set of sequential core allocations change as the set of available assets expands?

(2) Under what conditions does the strong sequential core concide with the classical core?

(3) Are there asset structures such that the sequential core is always nom-empty?

The dynamic structure of a two-period economy is as follows. In period $t=0$, the players have the possibility to trade in assets. An asset is a contract that specifies how 
much of each commodity a holder of the asset should deliver or receive in each state. In the beginning of period $t=1$, the state of nature is revealed to the players, and the delivery of goods takes place, as specified by each player's portfolio of assets. After that, the players can engage in trade anew. Before the gane begins, the players should reach an agreenent as to how to trade in both periods.

In our treatment, assets represent, enforceable contracts. That is, no coalition of players can fail to deliwer goods as specified by the coalitional portfolio of assets. On the other hand, agreements as to the trade following the realization of the state of nature are not binding: players may deviate from such agrements after they learn the true state.

We show that the strong sequential core becomes larger as the set of available assets expands: The increase in the number of enforceable contracts enlarges the possibilities of the grand coalition, without altering the blocking power of the deviating coalitions. This result does not hold for the weak sequential core. In the weak sequential core the expansion of the set of enforceable agreements enlarges the possibilities of both the grand coalition and the deviating coalitions, often asymmetrically. Therefore, the weak sequential core may become empty after an additional asset has been introduced into the economy.

To answer the second question, we introduce the condition of strong completeness of the set of assets, which is a strengthening of the completeness condition as understood in general equilibrium theory. We establish a coexistence of the strong sequential core and the classical core for economies with a strongly complete set of assets. This result, however, crucially depends on the absence of any restrictions on the asset holdings of the players at the end of period $t=0$. If the commodity bundles obtained after the first round of trade are required to lie in the respective consumption sets, then the equivalence of the strong sequential core and the classical core fails.

A series of counterexamples shows that apart from some trivial special cases, nonemptiness of the strong (weak) sequential core in a two-period economy is difficult to guarantee. In Chapters 6 and 7 we consider the strong sequential core in the setting of a stationary economy (and stationary game, respectively) with an infinite time horizon. We show that, even in the absence of any enforceable contracts, the strong sequential core is non empty, provided that the discount factor is close enough to one. 


\section{Part I}

\section{Payoff-dependent Balancedness and the Core}





\section{Chapter 2}

\section{A Necessary and Sufficient Condition for the Non-emptiness of the Core of a Non-transferable Utility Game ${ }^{1}$}

\subsection{Introduction}

A cooperative game with non-transferable utility is an assignment of a set of feasible utility allocations to each coalition of players. The core of a cooperative game selects those utility allocations that are robust to all possible deviations by coalitions.

In Bondareva [7] and in Shapley [31] the notion of a balanced game has been introduced for games with transferable utility (TU-games). It has been demonstrated that a TU-game has a non-empty core if and only if it is a balanced game. Scarf [30] has extended the condition of balancedness to games with non-transferable utility (NTU-games) and proved that every balanced NTU-game has a non-empty core.

Billera [4] has further generalized the notion of a balanced game. In his work the batancedness condition is defined with respect to a system $\pi$ of coalitional vectors specifying the power or weight of each agent within every coalition. The existence of a system of coalitional vectors $\pi$ such that the game is $\pi$-balanced suffices for the core to be nonempty. Moreover, when attention is restricted to hyperplane games, a non-cmpty core implies $\pi$-balancedness for an appropriate choice of $\pi$. In general, the equivalence of $\pi$ balanced games and games with a non-empty core does not hold: an example of a game with a non-empty core that is not balanced for any choice of $\pi$ is given in Billera [4], Generalizations of Billera's balancedness conditions have been given for cooperative games in permutational structure (see van der Laan, Talman and Yang [33]) and cooperative games in graph structure (see Herings, van der Laan and Talman [13]). These conditions weaken $\pi$-balancedness and are sufficient for the core to be non-empty, though not necessary.

A natural generalization of the $\pi$-balancedness condition is achieved by allowing the system of coalitional vectors $\pi$ to depend on the utility allocations. In this chapter we

\footnotetext{
${ }^{1}$ This chapter is based on Predtetchinski and Herings [24].
} 
define the balancedness of the game with respect to a correspondence IT that assigns to each allocation of utilities a set of coalitional vectors $\pi$. We therefore allow the power of an agent within a coalition to depend on the utility allocation that is proposed. We prove that the core of an NTU-game is non-empty if it is balanced with respect to some correspondence $\Pi$. Conversely, for a given game with a non-empty core we construct a correspondence $\Pi$ satisfying the condition of $\Pi$-balancedness. Thus, $\Pi$-balancedness is not only a sufficient, but also a necessary condition for non-emptiness of the core.

To the best of our knowledge, the only alternative necessary and sufficient balancedness condition for non-emptiness of the core is given by Keiding and Thorlund-Petersen [16]. The advartage of our $\Pi[-$ balancedness condition over the condition in Keiding and Thorlund-Petersen [16] is that it applies directly to the game of interest and avoirds the construction of any anxiliary games or sequences of approximating games.

The so-called $\Psi$-balancedness condition introduced by Bonnisseau and Iehle $[8]$ is a payoff-dependent balancedness condition that further generalizes the $\Pi$-balancedness condition. It is not only a sufficient condition for the core to be non-empty, but also provides a non-empty selection from the core, called the $\Psi$-balanced core. The $\Psi$-balanced core will play an important role in the analysis of the fuzzy core in Chapter 3.

The outline of the chapter is as follows. In Section 2.2 some notation is introduced. In Section 2.3 we discuss the $\pi$-balancedness condition of Billera $[4]$ and the $(\pi,<)$ balancedness condition of Keiding and Thorlund-Petersen [16]. In Section 2.4 the $\Pi 1-$ balancedness condition is defined and the main result of the chapter is proved. In Section 2.5 we examine an example (due to Billera [4]) of a game which has a non-empty core without being $\pi$-balanced for any system of coalitional vectors $\pi$. In Section 2.6 the concept of $\Psi$-balanced core is discussed.

\subsection{Notation}

Wo summarize the notation to be used in this and the following chapter. Let $n$ be a positive integer. Then $N$ is the seti of integers $\{1, \ldots, n\}$, and $\mathcal{N}$ is the collection of non-empty subsets of the set $N$. $\mathbb{R}^{*}$ is the space of functions $x: i \in N \mapsto x^{i} \in \mathbb{R}$. $\mathbb{R}^{N}$ is the space of functions $\lambda: S \in \mathcal{N} \mapsto \lambda_{S} \in \mathbb{R}$. Define the set $\Delta_{N}=\left\{\pi_{N} \in \mathbb{R}^{20} \mid \pi_{N}^{i} \geq 0\right.$ for all $i \in$ $N$ and $\left.\sum_{i \in N} \pi_{N}^{i}=1\right\}$. Given $S \in \mathcal{N}$, define the set $\Delta_{S}=\left\{\pi_{S} \in \Delta_{N} \mid \pi_{S}^{i}=0\right.$ for all $i \in$ $N \backslash S\}$. Thus $\Delta_{N}$ is a unit. simplex in $\mathbb{R}^{n 2}$, and for each $S \in \mathcal{N}$ the set $\Delta_{S}$ is a face of $\Delta_{N}$. Denote the barycenter of $\Delta_{N}$ by $\beta$. Let $\Delta$ denote the product of the spaces $\Delta_{S}$ over all $S \in \mathcal{N}$. Given an $\epsilon \in(0,1 / n)$, let $\Delta_{S}=\left\{\pi_{S} \in \Delta_{S} \mid \pi_{S}^{i} \geq \epsilon\right.$ for all $\left.i \in S\right\}$. Let $\Delta_{N}$ denote the unit simplex in $\mathbb{R}^{N}, \Delta_{\mathcal{N}}=\left\{\lambda \in \mathbb{R}^{\mathcal{N}} \mid \lambda_{S} \geq 0 \forall S^{\prime} \in \mathcal{N}, \sum_{S \in \mathcal{N}} \lambda_{S}=1\right\}$. If $x$ and $y$ are For each $S \in \mathcal{N}$ let $1_{S}$ be the vector in $\mathbb{R}^{n}$ with component $1_{S}^{i}$ equal to 1 if $i \in S$ and equal the interior, the boundary, and the convex hull of $A$. 


\section{$2.3 \pi$-Balancedness and $(\pi,<)$-balancedness}

An $n$-person game with non-transferable utility (referred to throughout this chapter simply as a game) is a family of sets $V=\langle V(S)\rangle_{S E N}$ satisfying the conditions (G1)-(G3) below.

(G1) For all $S \in \mathcal{N}, V(S)$ is a non-empty proper closed subset of $\mathbb{R}^{n}$.

(G2) For all $S \in \mathcal{N},\left[x \in V(S), y \in \mathbb{R}^{n}, y^{i} \leq x^{i}\right.$ for all $\left.i \in S\right]$ implies $[y \in V(S)]$.

(G3) The set $V(N) \backslash \bigcup_{i \in N}$ int $V(\{i\})$ is non-empty and compact.

Sometimes the condition (G3) will be replaced by a stronger condition (G4):

(G4) There exists a number $M$ such that $v^{i} \leq M$ for all $S \in \mathcal{N}, v \in V(S)$, and $i \in S$.

A vector $x \in \mathbb{R}^{n}$ is an element of the core of the game $V$ if $x \in V(N)$ and there exist no $S \in \mathcal{N}$ and no $y \in V(S)$ such that $x^{i}<y^{i}$ for all $i \in S$. The core of the game $V$ is a set of $\mathbb{R}^{n}$ defined by the equation

$$
C(V)=V(N) \backslash \bigcup_{S \in N} i n t V(S) .
$$

Below we reproduce the $\pi$-balancedness condition of Billera [4]. Let an elemont $\pi$ of the set $\Delta$ be given. A collection of coalitions $\beta \subseteq \mathcal{N}$ is said to be $\pi$-balanced if there exists $\lambda \in \Delta_{\mathcal{N}}$ such that

$$
\pi_{N}=\sum_{S \in \beta} \lambda_{S} \pi_{S}
$$

In this case $\lambda$ is said to be a vector of balancing weights. A game $V$ is said to be $\pi$-balanced if for every $\pi$-balanced collection of coalitions $\beta \subseteq \mathcal{N}$ it holds that

$$
\bigcap_{S \in G} V(S) \subseteq V(N)
$$

If there exists some $\pi \in \Delta$ such that the game $V$ is $\pi$-balanced, then the core of $V$ is non-empty.

The $\pi$-balancedness condition has been generalized in the work of Keiding and ThorlundPetersen [16]. Given a game $V$ and a coalition $S \in \mathcal{N}$ let $V_{p}(S)=\left\{x^{S} \in \mathbb{R}_{+}^{S} \mid x \in V(S)\right\}$, where $\mathbb{R}_{+}^{S}$ is the set of non-negative functions with domain $S$, and $x^{5}$ is the restriction of the function $x \in \mathbb{R}^{n}$ to the set $S .^{2}$ Let $\mathcal{R}$ denote the set of binary relations $<$ on the set $\mathcal{N}$ satisfying the following conditions:

- < is acyclic: If $S_{j} \in \mathcal{N}$ for all $j \in\{1, \ldots, k\}$ and $S_{1}<S_{2}<\ldots<S_{k}$, then we cannot have $S_{k}<S_{1}$

\footnotetext{
${ }^{2}$ Tn the paper of Keiding and Thorlund-Petersen [16] a somewhat different definition of a nontransferable utiliby game is given. In particular, the set $V(S)$ is assumed to lie entirely in the non negative orthant of $\mathbb{R}^{n}$ and to contain the zero vector. The set $V_{p}(S)$ is assumed to be bounded for all $S \in \mathcal{N}$.
} 
- all one player coalitions are minimal: For all $i \in N$ there is no $S \in \mathcal{N}$ such that $S<\{i\}$.

Let $\pi \in \Delta$ and $<\in \mathcal{R}$ be given. A game $V$ is said to be $(\pi,<)$-balanced if the following condition is satisfied: If $\beta \subseteq \mathcal{N}$ is a $\pi$-balanced collection of coalitions and

$$
x \in \bigcap_{S \in \beta} V(S) \backslash \bigcup_{Q \in N, Q} i n t V(Q),
$$

then $x \in V(N)$. The game $V$ is said to be weakly $(\pi,<)$-balanced if there exists a sequence $\left\{V^{*}\right\}_{\tau=1}^{\infty}$ of $(\pi,<)$-balanced games such that

- $V(N)=V^{r}(N)$ for all $\tau$, and

- for every $S \in \mathcal{N}$ the sequence $\left\{V_{p}^{\tau}(S)\right\}_{\tau=1}^{\infty}$ converges to the set $V_{p}(S)$ in the topology induced by the Hausdorff metric on the set of non-empty compact subsets of $\mathbb{R}^{n}$.

Theorem 2.1 below gives a characterization of NTU-games with a non-empty core.

Theorem 2.1 (Keiding and Thorlund-Petersen, 1987) Let $V$ be a game. Then the core of $V$ is non-empty if and only if there exist $\pi \in \Delta,<\in \mathcal{R}$, and a weakly $(\pi,<)$ balanced game $V^{\prime}$ such that $V(N)=V^{\prime \prime}(N)$ and $V(S) \subseteq V^{\prime}(S)$ for all $S \in \mathcal{N}$.

The purpose of the next section is to give a necessary and sufficient condition for nonamptiness of the core that applies directly to the game of interest $V$. In particular, our condition of $\Pi$-balancedness avoids the construction of an auxiliary game like $V^{\prime}$ and does not use the approximation of the game $V^{r}$ by a sequence of games $\left\{V^{r}\right\}_{r=1}^{\infty}$.

\section{$2.4 \quad \Pi$-Balancedness}

Definition 2.1 Let $V$ be a game. Let $\Pi: \mathbb{R}^{n} \rightarrow \Delta$ be a correspondence with non-empty convex values and with a closed graph. The game $V$ is said to be IT-balanced provided that the following condition is satisfied: If $x \in \mathbb{R}^{n}, \pi \in \Pi(x)$, and $\lambda \in \Delta_{N}$ are such that

then $x \in V(N)$.

$$
\begin{aligned}
& x \in \bigcap_{S \in \mathcal{N}} V\left(\lambda_{S}>0\right. \\
& \pi_{N}=\sum_{S \in \mathcal{N}} \lambda_{S} \pi_{S},
\end{aligned}
$$

The $\pi$-balancedness condition of Billera [4] is defined relative to a fixed element $\pi$ of the set $\Delta$. It corresponds to the special case of Definition 2.1 where $\Pi$ is single-valued and independent of the utility allocation. By using the concept of $1 \mathrm{~T}$-balancedness, we allow the power of an agent within a coalition to depend on the utility allocation that is proposed. 
Theorem 2.2 Let $V$ be a game satisfying conditions (G1) (G3). Suppose that there exists a correspondence $\mathbb{I}: \mathbb{R}^{n} \rightarrow \Delta$ with non-empty convex values and with a closed graph such that the game $V$ is $\Pi$-balanced. Then the core of the game $V$ is non-empty.

The proof of Theorem 2.2 is postponed until Section 2.6 , where a more general proposition will be established. The proof of Theorem 2.3 below is straightforward and therefore omitted.

Theorem 2.3 Let $V$ be a game satisfying conditions $(\mathrm{G} 1)-(\mathrm{G} 3)$ and let $S \in \mathcal{N}$. Then

1. for every $x \in \mathbb{R}^{n}$ there exists a unique real number $t_{S}(x)$ such that $\left(x-t_{S}(x) 1_{S}\right) \in$ $\partial V(S)$;

2. the function $t_{S}: x \mapsto t_{S}(x)$ is a continuous function;

3. $x \in V(S)$ if and only if $t_{S}(x) \leq 0 ; x \in \mathbb{R}^{n} \backslash \operatorname{int} V(S)$ if and only if $t_{S}(x) \geq 0$.

Given a game $V$, define a map $g_{S}: \mathbb{R}^{n} \rightarrow \partial V(S)$ by $g_{S}(x)=x-t_{S}(x) \mathbf{1}_{S}$ for all $x \in \mathbb{R}^{n \prime}$. Observe that $g_{S}$ is a continuous function that restricts to the identity map on $\partial V(S)$.

Theorem 2.4 below asserts that ПI-balancedness is not only a sufficient, but also a necessary condition for the non-emptiness of the core.

Theorem 2.4 Let $V$ be a game satisfying conditions (G1)-(G3). Suppose that the core of $V$ is non-empty. Then there exists a correspondence $\Pi: \mathbb{R}^{n} \rightarrow \Delta$ with non-empty convex values and with a closed graph such that the game $V$ is $\Pi$ I-balanced.

Proof. Let $x^{*}$ be an element of the core of the game $V$. Define the correspondence II : $\mathbb{R}^{n} \rightarrow \Delta$ by the equations

$$
\begin{gathered}
\Pi(x)=\underset{S \in \mathcal{N}}{x} \Pi_{S}(x) . \\
\Pi_{S}(x)=\left\{\pi_{S} \in \Delta_{S} \mid \pi_{S} \cdot g_{S}\left(x^{*}\right)=\pi_{S} \cdot g_{S}(x)\right\}
\end{gathered}
$$

for all $x \in \mathbb{R}^{n}$.

To see that the set $\prod_{S}(x)$ is non-empty, observe that it is given by the intersection of the sets $\mathbb{\Pi}_{S}^{-}(x)$ and $\Pi_{S}^{+}(x)$, where

$$
\begin{aligned}
& \Pi_{S}^{-}(x)=\left\{\pi_{S} \in \Delta_{S} \mid \pi_{S} \cdot g_{S}\left(x^{*}\right) \leq \pi_{S} \cdot g_{S}(x)\right\} . \\
& \Pi_{S}^{+}(x)=\left\{\pi_{S} \in \Delta_{S} \mid \pi_{S} \cdot g_{S}\left(x^{*}\right) \geq \pi_{S} \cdot g_{S}(x)\right\} .
\end{aligned}
$$

If the set $\Pi_{S}^{-}(x)$ were empty for some $x \in \mathbb{R}^{n}$, then the inequality $\pi_{S} \cdot g_{S}\left(x^{*}\right)>\pi_{S} \cdot g_{S}(x)$ would be satisfied for all $\pi_{S} \in \Delta_{S}$. In particular, this inequality would hold for all vertices of the simplex $\Delta_{S}$, implying that $g_{S}^{i}\left(x^{*}\right)>g_{S}^{i}(x)$ for all $i \in S$. As $g_{S}\left(x^{*}\right) \in \partial V(S)$, this would imply $g_{S}(x) \in \operatorname{in} t V(S)$, contradicting the definition of 9.5 . A similar argument shows that the set $\Pi_{S}^{+}(x)$ is non-empty. Moreover, both sets $\Pi_{S}^{-}(x)$ and $\Pi_{S}^{+}(x)$ are closed, and $\Pi_{S}^{-}(x) \cup \Pi_{S}^{+}(x)=\Delta_{S}$. Connectedness of the set $\Delta_{S}$ implies that the intersection of $\Pi_{S}^{-}(x)$ and $\Pi_{S}^{+}(x)$ is non-empty. 
Convexity of the sets $\Pi_{S}(x)$ is trivial.

To prove that the graph of the correspondence $\Pi$ is closed, let $\left(x_{(k)}, \pi_{(k)}\right)$ be a sequence of points in $\mathbb{R}^{n} \times \Delta$ satisfying $\pi_{(k)} \in \Pi\left(x_{(k)}\right)$ and converging to some point $\left(x_{(0)}, \pi_{(0)}\right)$. Then the equalities

$$
\pi_{S(k)} \cdot g_{S}\left(x^{*}\right)=\pi_{S(k)} \cdot g_{S}\left(x_{(k)}\right), S \in \mathcal{N}
$$

hold for all members of the sequence. By continuity of the mappings $g_{S}$, it holds in the limit that:

$$
\pi_{S(0)} \cdot g_{S}\left(x^{*}\right)=\pi_{S(0)} \cdot g_{S}\left(x_{(0)}\right), S \in \mathcal{N} .
$$

Therefore, $\pi_{(0)} \in \Pi\left(x_{(0)}\right)$.

Next we show that the game $V$ is $\Pi$-balanced. Let $x \in \mathbb{R}^{n}, \pi \in \Pi(x)$, and $\lambda \in \Delta_{\mathcal{N}}$ be such that

$$
\begin{gathered}
x \in \bigcap_{S \in N} V(S) \\
\pi_{N}=\sum_{S \in \mathcal{N}} \lambda_{S} \pi_{S} .
\end{gathered}
$$

By part (3) of Lemma 2.3, for all $S \in \mathcal{N}$ with $\lambda_{S}>0$ it holds that $t_{S}(x) \leq 0$. As $x^{*} \notin \operatorname{int} V(S)$, we have $0 \leq t_{S}\left(x^{*}\right)$. It follows that for all $S \in \mathcal{N}$ with $\lambda_{s}>0$ the following inequalities hold:

$$
\begin{aligned}
\pi_{S} \cdot x & \leq \pi_{S} \cdot g_{S}(x) \\
& =\pi_{s} \cdot g_{S}\left(x^{2}\right) \\
& \leq \pi_{S} \cdot x^{*}
\end{aligned}
$$

Therefore,

$$
\begin{aligned}
\pi_{N} \cdot x & =\sum_{\operatorname{SEN}_{:} \lambda_{S}>0} \lambda_{S} \pi_{S} \cdot x \\
& \leq \sum_{S_{E N}: \lambda_{S}>0} \lambda_{S} \pi_{S} \cdot x^{*} \\
& =\pi_{N} \cdot x^{*} \\
& =\pi_{N} \cdot g_{N}\left(x^{*}\right) \\
& =\pi_{N} \cdot g_{N}(x),
\end{aligned}
$$

where the second to last equality is implied by the fact that $x^{*}=g_{N}\left(x^{*}\right)$. By definition of $g_{N}(x)$, this gives the inequality $t_{N}(x) \leq 0$, and the inclusion $x \in V(N)$.

Theorems 2.2 and 2.4 show that $\Pi$-balancedness is necessary and sufficient for nonemptiness of the core. The condition is relatively simple and provides a natural extension of $\pi$-balanced concept of Billera that closes the gap between necessary and sufficient con-
ditions for non-emptiness of then 


\subsection{An example}

In this section we examine the example from Billera [4] of a game which has a non-empty core without being: $\pi$-balanced for any system of coalitional vectors $\pi$. We show that there is a natural choice of the correspondence II such that the game in question is M-balanced. In addition, we demonstrate that the game is not $(\pi,<)$-balanced for any $\pi$ and any binary operation <, and construct an artificial game $V^{\prime}$ satisfying the condition of Theorem 2.1.

Let $V$ be a game with three players where

$$
\begin{aligned}
& V(\{1,2,3\})=\left\{x \in \mathbb{R}^{3} \mid x^{1} \leq 1, x^{2} \leq 1, x^{3} \leq 0\right\} \\
& V(\{1,2\})=\left\{x \in \mathbb{R}^{3} \mid x^{1}+x^{2} \leq 2\right\} \\
& V(S)=\left\{x \in \mathbb{R}^{3} \mid x^{i} \leq 0 \text { for all } i \in S\right\} \text { for any } S \neq N,\{1,2\} \text {. }
\end{aligned}
$$

The game $V$ has a unique core-element, $(1,1,0)$.

We show that there is no $\pi \in \Delta$ such that $V$ is a $\pi$-balanced game. Suppose first that the vector $\pi_{N}$ has at least one zero component. Then the collection of singletons $\beta=$ $\left\{\{i\} \mid \pi_{N}^{i}>0\right\}$ is a $\pi$-balanced collection of coalitions, whereas the intersection $\bigcap_{S \in A} V(S)$ is not a subset of $V(N)$.

Suppose that all components of the vector $\pi$ are positive. We can then assume without, loss of generality that $\pi_{N}^{i}=\frac{1}{n}$ for all $i \in N$. Now, if $\pi_{\{1,2\}}^{1}=\pi_{\{1,2\}}^{2}$ then the collection of coalitions $\alpha=\{\{1,2\},\{3\}\}$ is $\pi$-balanced. Since the vector $(2,0,0)$ is an element of $V(\{1,2\})$ and $V(\{3\})$ but not of $V(\{1,2,3\})$, the condition of $\pi$-balancedness is violated. In case $\pi_{\{1,2\}}^{1}>\pi_{\{1,2\}}^{2}$ the collection $\beta=\{\{1,2\},\{2\},\{3\}\}$ is $\pi$-balanced, with $(2,0,0)$ being feasible for each $S$ in $\beta$. Finally, if $\pi_{\{1,2\}}^{1}<\pi_{\{1,2\}}^{2}$ then the collection of coalitions $\gamma=$ $\{\{1,2\},\{1\},\{3\}\}$ is $\pi$-balanced. In this case the vector $(0,2,0)$ violates the $\pi$-balancedness condition.

Having these considerations in mind it is straightforward to construct a correspondence $\Pi$ such that the game $V$ is $\Pi$-balanced. In particular, there should be no $\pi$ in $\Pi(2,0,0)$ such that either $\alpha$ or $\beta$ is a $\pi$-balanced collection of coalitions. There also should bo no $\pi \in \Pi(0,2,0)$ such that the collection $\gamma$ is balanced relative to $\pi$. We let

$$
\Pi_{\{1,2\}}(x)= \begin{cases}\{(a, b, 0)\} & \text { if } x^{1}<x^{2}, \\ \Delta_{\{1,2\}} & \text { if } x^{1}=x^{2} \\ \{(b, a, 0)\} & \text { if } x^{1}>x^{2}\end{cases}
$$

where the numbers $a$ and $b$ satisfy $a>b \geq 0$ and $a+b=1$. For any coalition $S \neq\{1,2\}$ we let $\Pi_{s}(x)$ equal the barycenter of $\Delta_{s}$.

To see that $V$ is balanced relative to $\Pi$ let $x \in \mathbb{R}^{n}, \pi \in \Pi(x)$, and $\lambda \in \Delta_{\mathcal{N}}$ be such that

$$
\begin{aligned}
& x \in \bigcap_{S \in S} V(S), \\
& \pi_{N}=\sum_{S \in \mathcal{N}} \lambda_{S} \pi_{S},
\end{aligned}
$$


where $\beta=\left\{S \in \mathcal{N}: \lambda_{S}>0\right\}$. If $N \in \beta$ there is nothing to prove. We therefore assume that $N \notin \beta$. Suppose also that the coalition $\{1,2\}$ is not a member of the collection $\beta$. Since $\pi_{N}^{l}>0, \beta$ must contain either the coalition $\{1\}$ or the coalition $\{1,3\}$, or both. In aither case $x^{1} \leq 0$. Similar reasoning for $x^{2}$ and $x^{3}$ shows that none of them is positive. Hence, $x \in V(N)$.

Now consider the case where the coalition $\{1,2\}$ is a member of $\beta$. If $x^{1}<x^{2}$ then, by construction of the correspondence $\Pi, \pi_{\{1,2\}}^{1}>\pi_{\{1,2\}}^{2}$. Therefore, the collection $\beta$ must contain either coalition $\{2\}$ or coalition $\{2,3\}$, or both, so $x^{2} \leq 0$. Since $\pi_{N}^{3}>0$, there is at least one coalition in $\beta$ which contains player 3 , so that $x^{3} \leq 0$. Thus, $x$ belongs to $V(N)$. If $x^{2}<x^{1}$ then $\pi_{\{1,2\}}^{2}>\pi_{\{1,2\}}^{1}$ implying that $\beta$ contains either the singleton $\{1\}$ or the coalition $\{1,3\}$. In both cases, $x^{1} \leq 0$. As before, we have $x^{3} \leq 0$. Thus, $x \in V(N)$. Finally, if $x^{1}$ equals $x^{2}$ then both of them are less than or equal to 1 , and therefore $x$ belongs to $V(N)$.

Thrning to the condition of Keiding and Thorlund-Petersen, observe that the vectors $(2,0,0)$ and $(0,2,0)$ are not in the interior of any of the feasibility sets $V(S)$ of the game $V$. Hence, our argument that $V$ is not a $\pi$-balanced game for any $\pi$ is valid as a proof that it is not a $(\pi,<)$-balanced game for any $\pi$ and any $<\in \mathcal{R}$. Let $V^{\prime}$ be a game where

$$
V^{\prime}(\{i\})=\left\{x \in \mathbb{R}^{3} \mid x^{i} \leq 1\right\} \text { for } i=1,2,
$$

and $V^{\prime \prime}(S)=V(S)$ for all other coalitions $S$. Choose $\pi$ in $\Delta$ so that $\pi_{N}^{i}>0$ for all $i \in N$. Let $<\in R$ be a binary relation satisfying $\{i\}<\{1,2\}$ for $i=1,2,3$. Then $V^{\prime}$ is a $(\pi,<)$ balanced game. Indeed, let $\beta$ be a $\pi$-balanced collection of coalitions and let $x$ be an element of the set:

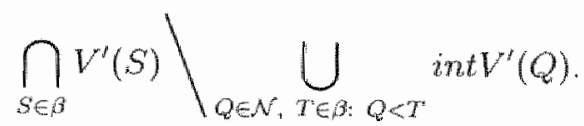

If the coalition $\{1,2\}$ is not in $\beta$ then one can argue as in the case with $I T$-balancedness that $x \in V^{\prime}(N)$. If $\{1,2\}$ is a member of the collection $\beta$ then $x$ must lie outside the interior of $V^{\prime}(\{\})$ for all $i \in N$, which implies that $x^{1} \geq 1, x^{2} \geq 1$, and $x^{3} \geq 0$. Thus, $x=(1,1,0)$, which belongs to $V^{\prime}(N)$.

\subsection{The $\Psi$-Balanced core}

This section continues the discussion of payolf-dependent balancedness in games. We consider the concept of the $\Psi$-balanced core, a refinement of the core introduced recently in the work of Bonnisseau and lehle $[8]^{3}$. The main result of this section states that the $\Psi$-balanced core of a game is mon-empty, provided that the game satisfies the $\Psi$ balancedness condition. As any $\Pi[$-balanced game is also $\Psi$-balanced, we obtain Theorem 2.2 as a corollary.

\footnotetext{
"In Bonnisseu and lehle $[8]$ it is called the "core with additional reguirements." We find it more convenicnt to refer to this solution concept as $\Psi$ balanced core.
} 
Definition 2.2 Let $V$ be a game. Let $\Psi: \mathbb{R}^{n} \rightarrow \Delta \times \Delta_{N}$ be a correspondence with non-empty convex values and with a closed graph. Then the $\Psi$-balanced core of the game $V$ is the set of points $x$ in the core of the game $V$ such that there exist $(\pi, \phi) \in \Psi(x)$ and $\lambda \in \Delta_{N}$ satisfying

$$
\begin{gathered}
x \in \bigcap_{S \in \mathcal{N}: \lambda_{S}>0} V(S), \\
\phi=\sum_{S \in \mathcal{N}} \lambda_{S} \pi_{S} .
\end{gathered}
$$

Thus the $\Psi$-balanced core is a selection from the core. If for each $x \in \mathbb{R}^{n}$ there exists a $(\pi, \phi) \in \Psi(x)$ such that $\pi_{N}=\phi$, then the $\Psi$-balanced core of the game $V$ coincides with the core of $V$. The $\Psi$-balanced core is a generalization of the socially stable core in Herings, van der Laan, and Talman [13].

Definition 2.3 Let $V$ be a game. Let $\Psi: \mathbb{R}^{r} \rightarrow \Delta \times \Delta_{N}$ be a correspondence with non-empty convex values and with a closed graph. The game $V$ is said to be $\Psi$-balanced provided that the following condition is satisfied: If $x \in \mathbb{R}^{n},(\pi, \phi) \in \Psi(x)$, and $\lambda \in \Delta_{N}$ are such that

$$
\begin{gathered}
x \in \bigcap_{S \in \mathcal{N}: \lambda_{S}>0} V(S), \\
\phi=\sum_{S \in N} \lambda_{S} \pi_{S},
\end{gathered}
$$

then $x \in V(N)$.

Any game balanced relative to the correspondence $\Pi: \mathbb{R}^{n} \rightarrow \Delta$ in the sense of Definition 2.1 is also balanced relative to the correspondence $\Psi: \mathbb{R}^{n} \rightarrow \Delta \times \Delta_{N}$ defined by the equation

$$
\Psi(x)=\left\{(\pi, \phi) \in \Delta \times \Delta_{N} \mid \pi \in \mathbb{M}(x) \text { and } \phi=\pi_{N}\right\},
$$

in the sense of Definition 2.3.

Bonmissean and lehlé $[8$. Theorem 2] prove that at $\Psi$-balanced game has a non mpty $\Psi$-balanced core. We reestablish this result with the help of the $\Psi$-balanced version of the Knaster, Kuratowski, Mazurkiewicz, and Shapley (K-K-M-S) theorem.

Theorem 2.5 (U-balanced weak $\mathbf{K}-\mathbf{K}-\mathbf{M}-\mathbf{S})$ Let $\left\{C_{S} \subseteq \Delta_{N} \mid S \in \mathcal{N}\right\}$ be a closed cover of $\Delta_{N}$ such that if $S$ and $T$ are elements of $\mathcal{N}$ and the set $\Delta_{T} \cap C_{S}$ is ron empty, then $S \subseteq T$. Let $\Psi: \Delta_{N} \rightarrow \Delta \times \Delta_{N}$ be a correspondence with non-empty convex values and with a closed graph. Then there exist $x^{*} \in \Delta_{N},\left(\pi^{*}, \phi^{*}\right) \in \Psi\left(x^{*}\right)$, and $\lambda^{*} \in \Delta_{\mathcal{N}}$ such that

$$
\begin{aligned}
& x^{*} \in \bigcap_{S \in \mathcal{N}_{i} \lambda_{S}^{*}>0} C_{S} \\
& \phi^{*}=\sum_{S \in \mathcal{N}} \lambda_{S}^{*} \pi_{S}^{*} .
\end{aligned}
$$


Theorem 2.5 can be easily established using the fixed point theorem of Kakutani, analogous to the proof of the $K-K-M-S$ theorem in Herings [12]. The original version of the $K-K-$ M-S theorem can be found in Shapley [32].

Theorem 2.6 Let $V$ be a game satisfying conditions (G1)-(G4). Let $\Psi: \mathbb{R}^{n} \rightarrow \Delta \times \Delta_{N}$ be a correspondence with ron-enpty convex vatues and with a closed graph. Then there exist $x^{*} \in \mathbb{R}^{n^{*}},\left(\pi^{*}, \phi^{*}\right) \in \Psi\left(x^{*}\right)$, and $\lambda^{*} \in \Delta_{\mathcal{N}}$ such that

$$
\begin{gathered}
x^{*} \in \bigcap_{S \in \mathcal{N}: \lambda_{S}>0} V(S) \backslash \bigcup_{T \in \mathcal{N}} \operatorname{int} V(T), \\
\phi^{*}=\sum_{S \in \mathcal{N}} \lambda_{S}^{*} \pi_{S}^{*} .
\end{gathered}
$$

The proof of Theorem 2.6 is very similar to that of the Scarf"s theorem in Kannai [15]. The only difference is that one has to use the $\Psi$-balanced version of the $K-K-M-S$ theorem stated above, rather than the standard version of this theorem.

Theorem 2.7 Let $V$ be a game satisfying conditions (G1)-(G3). Let $\Psi: \mathbb{R}^{n} \rightarrow \Delta \times \Delta_{N}$ be a correspondence with non-empty convex values and with a closed graph. Suppose that the game $V$ is $\Psi$-balanced. Then the $\Psi$-balanced core of the game $V$ is non-empty.

Proof. If the game $V$ satisfies additional condition (G4), then the result follows at once from Theorem 2.6. We now extend the theorem to a general NTU-game. Let $V$ and $\Psi$ be as in the statement, of the theorem. For an integer $q$, define the game
$V$ by letting

$$
V^{i}(S)=\left\{v \in V(S) \mid v^{i} \leq q \text { for all } i \in S\right\}
$$

It is easy to see that the game $V^{q}$ satisfies conditions (G1)-(G4). By Theorem 2.6, there exist $x^{q} \in \mathbb{R}^{n},\left(\pi^{q}, \phi^{q}\right) \in \Psi\left(x^{q}\right)$ and $\lambda^{q} \in \Delta_{\mathcal{N}}$ such that

$$
\begin{gathered}
x^{q} \in \bigcap_{S \in \mathcal{N}: \lambda_{S}^{q}>0} V^{q}(S) \backslash \bigcup_{T \in \mathcal{N}} \operatorname{int} V^{q}(T), \\
\phi^{q}=\sum_{S \in \mathcal{N}} \lambda_{S^{q}}^{q} \pi_{S}^{q} .
\end{gathered}
$$
By the $\Psi$ balancedness of the original game $V$, the wector $x^{q}$ is an element of $V(N)$.
Clearly, the sets $V^{q}(\{i\})$ and $V(\{i\})$ coincide for all $i$ whenever $q$ is greater than any of
the individually rational payoffs of the game $V$. Therefore, $x^{q}$ in the individually rational payoffs of the game $V$. Therefore, $x^{q}$ lies in the compact set

$$
V(N) \backslash \bigcup_{i \in N} \operatorname{int} V(\{i\})
$$


for all sufficiently large $q$. We can therefore assume without loss of generality that the sequence $\left(x^{q}, \pi^{q}, \phi^{q}, \lambda^{q}\right)$ converges to a point $(\bar{x}, \bar{\pi}, \bar{\phi}, \bar{\lambda})$ of $\mathbb{R}^{n} \times \Delta \times \Delta_{N} \times \Delta_{M}$. By the closedness of the feasibility sets of the game $V$, the point $\bar{x}$ lies in $V(N)$ and in $V(S)$ whenever $\bar{\lambda}_{S}>0$. It does not belong to the interior of ary set $V(S)$, for otherwise it would lie in the interior of $V^{q}(S)$ for all sufficiently large $q$. Thus $\bar{x}$ is the core-element of the game $V$. Since the graph of the correspondence $\Psi$ is closed, $(\bar{\pi}, \bar{\phi}) \in \Psi(\bar{x})$. Moreover, the equation

$$
\bar{\phi}=\sum_{S \in \mathbb{N}} \bar{\lambda}_{s} \tilde{\pi}_{S} .
$$

holds. Thus, $\bar{x}$ lies in the $\Psi$-balanced core of the game $V$.

As any $\Pi$-balanced game is also $\Psi$-balanced, Theorem 2.7 implies that any $\mathbb{1}$-balanced game has a non-empty core. We have thus established Theorem 2.2. 



\section{Chapter 3}

\section{The Fuzzy Core and the $\Psi$-Balanced Core $^{1}$}

\subsection{Introduction}

The fuzzy core was introduced by Aubin [1] as the set of allocations of a pure exchange economy that are robust to blockings by all fuzzy coalitions. To interpret a fuzzy coalition, one thinks of an economy as being comprised of several types of agents, with each type being represented by a continuum of identical individuals. The fuzzy coalition then specifies the number (the mass) of the participants of each type.

The fuzzy core is known to be non-empty under very general conditions. In particular, Florenzano [10, see Proposition 5.2.3, p. 115] establishes the non-emptiness of the fuzzy core in an infinite-dimensional production economy.

This chapter provides a new proof of the non-emptiness of the fuzzy core. The proof is based on the concept of the $\Psi$ balanced core for games without side payments introduced in Bonnisseau and Iehle [8] and discussed in the previous chapter. A given economy gives rise to a non-transferable utility game whose $\Psi$ balanced core corresponds, in a natural way, to the $\epsilon$-fuzzy core of the original economy. The non-emptiness of the fuzzy corc can therefore be deduced from the non-emptiness result for the $\Psi$-balanced core provided in Section 2.6 .

Our non-emptiness result is weaker than that given in Proposition 5.2 .3 in Florenzano [10]. The result in Florenzano [10] applies to an econony with a non-trivial production sector, while our construction is limited to pure exchange economies. Furthermore, to transform the problem of the non-emptiness of the fuzzy core into that of the nonemptiness of the $\Psi$-balanced core, we have to rely on the existence of the utility functions. This is in contrast to the result in Florenzano [10], where the consumer preferences may lack completeness or transitivity. On the other hand, we are able to maintain quite general assumptions with respect to the commodity space of an economy. As in Florenzano $[10]$, the commodity space in this chapter is a general (possibly infinite dimensional) linear

\footnotetext{
${ }^{1}$ This chapter is based on Predtetchinski [23].
} 
Hausdorff topological space.

The rest of the chapter is organized as follows. In Section 3.2 the fuzzy core and the $\epsilon$-fuzzy core are defined. In Section 3.3 the non-emptiness of the $\epsilon$-fuzzy core is demonstrated.

\subsection{Fuzzy core}

We consider a pure exchange conomy $\mathscr{E}$ in which the set of agents is $N$, and the commodity space is $\mathrm{C}$. Each agent $i \in N$ is characterized by a consumption set $X^{i} \subseteq \mathrm{C}$, initial endowment of commodities $e^{i} \in \mathrm{C}$, and a utility function $u^{i}: X^{i} \rightarrow$ 俄. Given $S \in \mathcal{N}$ we write $X_{S}$ to denote the product $\underset{i \in S}{\times} X^{i}$.

We employ the following assumptions:

(A1) The commodity space $\mathrm{C}$ is a linear (over the field of real numbers) Hausdorff topological space.

(A2) For each $i \in N$, the set $X^{i}$ is a closed convex set containing $e^{i}$, and the set $F(\mathscr{E})=$ $\left\{x \in X_{N} \mid \sum_{i \in N} x^{i}=\sum_{i \in N} e^{i}\right\}$ of feasible commodity allocations is a compact set.

(A3) For each $i \in N$ the utility function $u^{i}$ is a continuous and quasi-concave function.

The fuzzy core of the economy $\mathscr{E}$ is the set of aliocations in $F(E)$ that are robust to deviations by all fuzzy coalitions. To interpret a fuzzy coalition, one thinks of an economy $\mathscr{E}$ as consisting of $n$ types of agents, with each type being represented by a contimuum (say by the unit interval $[0,1]$ ) of identical individuals. Each individual of type $i \in N$ has the consumption set $X^{i}$, the utility function $u^{i}$ and the initial endowment $e^{i}$. A fuzzy coalition then specifies the mass $\pi^{i}$ of the participants of type $i$, for each $i \in N$. A standard coalition $S \in \mathcal{N}$ is also a fuzzy coalition that contains all individuals of each type $i \in S$ and none of types $j \in N \backslash S$.

An assumption embodied in the definition of the fuzzy core is that in any fuzzy coalition, in particular in the grand coalition $N$, individuals of the same type receive the same commodity bundle. This assumption can be justified by modelling the economy $\mathscr{E}$ explicitly as an economy with a continum of traders as is done in Hüsseinov [14], and demonstrating that in any Pareto efficient allocation the equal treatment property holds. With the assumption of equal treatment, the set of feasible allocations for a fuzzy coalition $\pi$ is determined by the equation $\sum_{i \in N} \pi^{i} x^{i}=\sum_{i \in N} \pi^{i} e^{i}$. Since this feasibility condition is linearly homogeneous in $\pi$, we can nomalize the vector $\pi$ so that it becomes an element. of the unit simplex $\Delta_{N}$. A standard coalition $S$ is then identified with the barycenter of
the simplex $\Delta_{S}$.

Definition 3.1 An allocation $x \in F(\mathscr{E})$ is an elemeat of the fuzzy core $C^{f}(\mathscr{E})$ of the economy $\mathscr{E}$ if there exist no $\pi \in \Delta_{N}$ and no $\chi \in X_{N}$ such that $\sum_{i \in N} \pi^{i} \chi^{i}=\sum_{i \in N} \pi^{i} e^{i}$ and
$\left.u^{i}\right)>u^{i}\left(x^{i}\right)$ for all $i \in N$ with $\pi^{i}>0$ 
It is instrumental to consider the following modification of the fuzzy core, called the - -fuzzy core. In the $\epsilon$-fuzzy core it is required that for any fuzzy coalition $\pi$ with support $S$ the mass $\pi^{2}$ of the participants of each type $i \in S$ be at least $c>0$.

Definition 3.2 An allocation $x \in F(\mathscr{E})$ is an element of the $\epsilon$ fuzzy core $C f(\mathscr{f})$ of the economy $\mathscr{E}^{\mathbb{E}}$ if there exist no $S \subseteq N$, no $\pi \in \Delta_{S}^{*}$ and no $\chi \in X_{S}$ such that $\sum_{i \in S^{\pi}} \pi^{i}=$ $\sum_{i \in S} \pi^{i} e^{i}$ and $u^{i}\left(\chi^{i}\right)>u^{i}\left(x^{i}\right)$ for all $i \in S$.

Theorem 3.1 For each $\epsilon \in(0,1 / n)$, the $\epsilon$ fuzzy core of the economy $\mathscr{E}^{2}$ is non-empty.

The proof of Theorem 3.1 is given Section 3.3. Our main result is the following.

Theorem 3.2 The fuzzy core of the economy $\mathscr{B}^{\circ}$ is non-empty.

Proof. To prove Theorem 3.2 we consider fuzzy core as a limit of the $\epsilon$-fuzzy core as $\epsilon$ approaches zero. Observe that $C_{\dot{f}}^{f}(\mathscr{E}) \subseteq C_{\epsilon^{\prime}}^{f}(\mathscr{E})$ whenever $0<\epsilon \leq \epsilon$. Moreover, the fuzzy core of the economy $\mathscr{E}$ can be written as

$$
C^{f}(\mathscr{E})=\bigcap_{\in \in(0,1 / p)} C_{f}^{f}(\mathscr{E})
$$

Since the non-emptiness of the 6 -fuzzy core follows from Theorem 3.1, it suffices to demonstrate that $C_{i}^{f}(\mathscr{E})$ is a compact set. We show that it is a closed subset of $F(\mathscr{E})$.

Let $x$ be a point of $F(\mathscr{E})$ not in $C_{\varepsilon}^{f}(\mathscr{E})$. Then there exist $S \subseteq N, \pi \in \Delta_{S}$ and $\chi \in X_{S}$ as in Definition 3.2. For a fixed $\chi$, the inequalities $u^{i}\left(\chi^{i}\right)>u^{i}\left(x^{i}\right), i \in S$, define an open neighborhood of $x$ in $F(\mathscr{E})$ contained entirely in the complement of $C_{\epsilon}^{f}(\mathscr{E})$. Thus, the complement of $C_{C}^{\mathcal{C}}\left(\mathscr{E}^{\circ}\right)$ in $F(\mathscr{E})$ is an open set, as desired.

\subsection{The non-emptiness of the $\epsilon$-fuzzy core}

In this section we prove Theorem 3.1. The main instrument of the proof is the concept of the $\Psi$-balanced core.

Given an $\in \in(0,1 / n)$ and an economy $\mathscr{B}$, we construct, a non-transferable utility game $V^{c}$ and the correspondence $\Psi$ in such a way that the $\Psi$-balanced core of the game $V^{\prime}$ corresponds, in a natural way, to the $\epsilon$-fuzzy core of the economy $E^{*}$. In the game $V^{c}$, the utility tuple ${ }^{2} v$ is considered feasible for a coalition $S$ if it is feasible for some fuzzy conlition $\pi_{S}$ with support $S$ in the original economy $\mathscr{E}$. The correspondence $\Psi: \mathbb{R}^{n_{2}} \rightarrow \Delta \times \Delta_{N}$ has a product structure, with the component that maps to $\Delta_{S}$ denoted by $\Pi_{S}$. Given the vector of utilities $v$ in $\partial V^{t}(S)$, we define the set $\Pi_{S}(v)$ to consist of those fuzzy coalitions $\pi_{S}$ that can achieve the utility of $v$. We then show that the the game $V^{\prime}$ is $\Psi$-balanced

\footnotetext{
${ }^{2}$ Note that we reserve the symbol $x$ to denote commodity allocations, and we use the symbol $y$ to denote utility tuples.
} 
and that its $\Psi$-balanced core corresponds, under the utility functions, to the $\epsilon$-fuzzy core of the economy $\mathscr{B}^{\circ}$.

Given $S \in \mathcal{N}$, let

$$
\begin{gathered}
Z^{e}(S)=\left\{\left(x_{S}, \pi_{S}\right) \in X_{S} \times \Delta_{S}^{e} \mid \sum_{i \in S^{i}} \pi_{S}^{i} x_{S}^{i}=\sum_{i \in S} \pi_{S}^{i} e^{i}\right\} \\
V^{\varepsilon}(S)=\left\{v \in \mathbb{R}^{n} \mid \begin{array}{c}
\text { there exists }\left(x_{S}, \pi_{S}\right) \in Z^{e}(S) \text { such that } \\
u^{i}\left(x_{S}^{i}\right) \geq v^{i} \text { for all } i \in S
\end{array}\right\} .
\end{gathered}
$$

Proposition 3.1 For each $\in(0,1 / n), Z^{e}(S)$ is a non-empty and compact set.

Proof. For each $\epsilon \in(0,1 / n)$, the set $\Delta_{S}^{g}$ is non-empty, and the set $Z^{\prime \prime}(S)$ contains a non-empty subset $\left\{e_{S}\right\} \times \Delta_{S}^{c}$, where $e_{S}^{i}=e^{i}$ for all $i \in S$. Define $F_{S}(\mathscr{E})$ to be the set of feasible conmodity allocations of the coalition $S$ :

$$
F_{S}(\mathscr{E})=\left\{x_{S} \in X_{S} \mid \sum_{i \in S} x_{S}^{i}=\sum_{i \in S} e^{i}\right\}
$$

First we argue that $F_{S}(\mathscr{E})$ is a compact set. The set $F_{N}(\mathscr{E})$ is compact by assumption (A2). For a a proper subset $S$ of $N$ the set $F_{S}(\mathscr{E})$ can be considered a subset of $F_{N}(\mathscr{E})$ given by

$$
F_{N}(\mathscr{E}) \bigcap\left\{x \in X \mid x^{i}=e^{i} \text { for all } i \in N \backslash S\right\} .
$$

By assumption (A2), $\mathrm{C}$ is a Hausdorff space, so a one-point set $\left\{e^{i}\right\}$ is closed in $X^{i}$ for each $i$. It follows that $F_{S}(\mathscr{E})$ is closed in $F_{N}(\mathscr{E})$; therefore, it is a compact set.

To see that $Z^{r}(S)$ is a compact set, let $|S|$ be the candinality of $S$; let $C^{|S|}$ be the $|S|$ fold product of $\mathbf{C}$, and let $\varphi$ be a contimuous function from the space $C^{|S|} \times \Delta_{S}^{\epsilon}$ to itself that carries a point $\left(x_{s}, \pi_{s}\right)$ to a point $\left(\widetilde{x}_{S}, \pi_{S}\right)$, where

$$
\widetilde{x}_{S}^{i}=\frac{1}{\pi_{S}^{i}} x_{S}^{i}+\left(1-\frac{1}{\pi_{S}^{i}}\right) e^{i}
$$

for each $i \in S$. Then

$$
Z^{\prime}(S)=\left(x_{S} \times \Delta_{S}^{e}\right) \bigcap \varphi\left(F_{S}(\mathscr{E}) \times \Delta_{S}^{e}\right)
$$

The product of the compact sets $F_{S}(\mathscr{E})$ and $\Delta_{S}^{E}$ is a compact set, and so is its image under $\varphi$. Thus $Z^{*}(S)$ is a closed subset of a compact set; therefore, it is a compact set.

Proposition 3.2 For each $\epsilon \in(0,1 / n)$, the family of sets $V^{6}=\left\langle V^{e}(S)\right\rangle_{S \in \mathcal{N}}$ satisfies conditions (G1), (G2) and (G4). 
Proof. Condition (G2) is satisfed by definition of $V^{c}(S)$. To verify conditions (G1) and (G4), let us write the set $V^{E}(S)$ as

$$
V^{c}(S)=\left\{v \in \mathbb{R}^{n} \mid \begin{array}{c}
\text { there exists } \dot{v} \in \dot{V}^{\prime}(S) \text { such that } \\
\dot{v}^{i} \geq v^{i} \text { for all } i \in S
\end{array}\right\} \text {. }
$$

where $\dot{V}(S)$ is defined by

$$
\dot{V}(S)=\left\{v \in \mathbb{R}^{S} \mid \begin{array}{c}
\text { there exists }\left(x_{S}, \pi_{S}\right) \in Z^{c}(S) \text { such that } \\
u^{i}\left(x_{S}^{i}\right)=v^{i} \text { for all } i \in S
\end{array}\right\} .
$$

The set $\dot{V}^{\prime}(S)$ is the image of the set. $Z^{c}(S)$ under the composition of the maps

$$
X_{S} \times \Delta_{S} \longrightarrow X_{S} \longrightarrow \mathbb{R}^{S}
$$

where the first map is the natural projection, and the second map is a Cartesian product of the functions $u^{i}$ for $i \in S$. It follows that $\dot{V}^{\varepsilon}(S)$ is a non-empty compact subset of $\mathbb{R}^{S}$; in particular, it is bounded from above. From this one can easily see that the set $V(S)$ satisfies conditions (G1) and (G4).

We remark that for $\epsilon=0$ the family of sets $V^{c}$ may fail to meet conditions (G1) and (G4), or even a weaker condition (G3), since $Z^{0}(N)$ is not, generally, a compact set. It is to avoid this difficulty that the $\epsilon$-fuzzy cores and the corresponding games $V^{\epsilon}$ were introduced.

Define the correspondences $\Pi_{S}: \partial V^{c}(S) \rightarrow \Delta_{S}^{e}$ as follows. Given $v \in \partial V^{c}(S)$ let

$$
\Pi_{S}(v)=\left\{\begin{array}{l|c}
\pi_{S} \in \Delta_{S} & \begin{array}{c}
\text { There exists } x_{S} \in X_{S} \text { such that } \\
\sum_{i \in S} \pi_{S}^{i} x_{S}^{i}=\sum_{i \in S} \pi_{S}^{i} e^{i} \\
u^{i}\left(x_{S}^{i}\right) \geq v^{i} \text { for all } i \in S
\end{array}
\end{array}\right\} .
$$

Proposition 3.3 for each $v \in \partial V^{c}(S)$ the set $\Pi_{S}(v)$ is non-empty and convex. The correspondence $\Pi_{S}$ has a closed graph.

Proof. The set $\Pi_{S}(v)$ is non-empty by definition of $V^{\prime}(S)$. To prove that it is a convex set, let $\pi_{S}, \pi_{S}^{\prime} \in \mathbb{I}_{S}(v)$ and $t \in[0,1]$ be given. We must. show that $\tilde{\pi}_{S}=t \pi_{S}+(1-t) \pi_{S}^{\prime}$ is an element $\Pi_{S}(v)$. Let $x_{S}, x_{S}^{\prime}$ be points in $X_{S}$ such that $\sum_{i \in S} \pi_{S}^{i} x_{S}^{i}=\sum_{i \in S} \pi_{S}^{i} e^{i}$ and $u^{i}\left(x_{S}^{i}\right) \geq v^{i}$ for all $i \in S$, and similarly for $x_{S}^{\prime}$. Given $i \in S$ let $\widetilde{x}_{S}^{i}$ be defined as

$$
\widetilde{x}_{S}^{i}=\frac{1}{\widetilde{\pi}_{S}^{i}}\left[t \pi_{S}^{i} x_{S}^{i}+(1-t) \pi_{S}^{\prime i} x_{S}^{\prime i}\right]
$$

By convexity of the consumption sets one has $\widehat{x}_{S}^{i} \in X^{i}$. By quasi-concavity of the utility 
function, $u^{i}\left(\tilde{x}_{S}^{i}\right) \geq v^{i}$ for all $i \in S$. Finally,

$$
\begin{aligned}
\sum_{i \in S} \tilde{\pi}_{S}^{i} \widetilde{x}_{S}^{i} & =t \sum_{i \in S} \pi_{S}^{i} x_{S}^{i}+(1-t) \sum_{i \in S} \pi_{S}^{i_{i}} x_{S}^{\prime i} \\
& =t \sum_{i \in S} \pi_{S}^{i} e^{i}+(1-t) \sum_{i \in S} \pi_{S}^{i n} e^{i} \\
& =\sum_{i \in S} \tilde{\pi}_{S}^{i} e^{i} .
\end{aligned}
$$

Thus, $\tilde{\pi}_{S} \in \Pi_{S}(u)$, as desired.

To see that the graph $G$ of the correspondence $\Pi_{S}$ is closed, let us write it as the set

$$
G=\left\{\left(u, \pi_{S}\right) \in \partial V^{c}(S) \times \Delta_{S} \mid \begin{array}{c}
\text { there exists }\left(\dot{v}, \pi_{S}\right) \in \dot{G} \\
\text { such that } \dot{v}^{i} \geq v^{i} \text { for all } i \in S
\end{array}\right\},
$$

where $\dot{G}$ is defined by

$$
\dot{G}=\left\{\left(v, \pi_{S}\right) \in \mathbb{R}^{S} \times \Delta_{S} \mid \begin{array}{c}
\text { there exists } x_{S} \in X_{S} \text { such that } \\
\left(x_{S}, \pi_{S}\right) \in Z^{\epsilon}(S) \\
u^{2}\left(x_{S}^{i}\right)=v^{i} \text { for all } i \in S
\end{array}\right\} .
$$

Then the set $G$ is the image of $Z^{c}(S)$ under the continuous map $X_{S} \times \Delta_{S}^{\varepsilon} \rightarrow \mathbb{R}^{S} \times \Delta_{S}^{*}$, which is a Cartesian product of the utility functions $u^{i}$ for $i \in S$ and the identity map on $\Delta_{S}$. It follows from Proposition 3.1 that $G$ is a compact set. From this one can easily see that the set $G$ is closed in $\partial V^{k}(S) \times \Delta_{S}^{t}$.

For each $S \in \mathcal{N}$, define the function $g_{S}: \mathbb{P}^{*} \rightarrow \partial V^{c}(S)$ as in Section 2.4 . Recall that $g_{S}$ is al continuous function that carries $\partial V^{c}(S)$ identically onto itself. The correspondence $\Pi_{S}$ : $\partial V(S) \rightarrow \Delta_{S}^{c}$ can now be extended to the whole of the space $\mathbb{R}^{n}$ taking the composition $\Pi_{S} \circ g_{s}$. Clearly, the extended correspondence has non-empty convex values and its graph is closed. We continue to denote the extended correspondence by $\Pi_{S}$.

Finally, we define the correspondence $\Psi: \mathbb{R}^{n} \rightarrow \Delta \times \Delta_{N}$ by the equation

$$
\Psi(v)=\underset{S \in M}{\times} \Pi_{S}(v) \times\{\beta\} .
$$

Theorem 3.3 Let the game $V^{*}$ and the correspondence $\Psi: \mathbb{R}^{n} \rightarrow \Delta \times \Delta_{N}$ be defined by Equations (3.1) (3.4). Let the allocation $x$ be an element of the e-fuzzy core of the economy 6 . Then the utility tuple $v$ is an element of the $\Psi$-balanced core of the game $V$, where $v^{i}=u^{i}\left(x^{i}\right)$ for all $i \in N$. Conversely, let the utility tuple $v$ be an element of the $\Psi$-balanced core of the game $V$. Then there exists a commodity allocation $x$ in the $\epsilon$-fuzzy core of the economy of such that $v^{i} \geq u^{i}\left(x^{i}\right)$ for all $i \in N$. 
Proof. Let the commodity allocation $x$ be in the $\epsilon-$ fuzzy core of the economy $\mathscr{E}$. Let $v \in \mathbb{R}^{n}$ be given by $v^{i}=u^{i}\left(x^{i}\right)$ for all $i \in N$. It is clear that $v$ is in the core of the game $V^{*}$. Since we have the inclusion $\beta \in \Pi_{N}(v)$, the vector of utilities $v$ is in the $\Psi$-balanced core of $V^{\epsilon}$.

Conversely, let the vector of utilities $v$ be in the $\Psi$-balanced core of the game $V *$. Then for each $S \in \mathcal{N}$ there exists a vector $\pi_{S} \in \Pi_{S}(v)$ and a non-negative number $\lambda_{S}$ such that

$$
\begin{gathered}
v \in \bigcap_{S \in \mathcal{N}: \lambda_{S}>0} V^{\epsilon}(S) \\
\beta=\sum_{S \in \mathcal{N}} \lambda_{S} \pi_{S} .
\end{gathered}
$$

The latter equation can be rewritten componentwise as

$$
1=\sum_{S \in \mathcal{N}} n \lambda_{S} \pi_{S}^{i} \text { for all } i \in N .
$$

It follows from the definition of the correspondences $\Pi_{S}$ that for every $S \in \mathcal{N}$ there exists a commodity allocation $x_{S} \in X_{S}$ such that

$$
\begin{gathered}
\sum_{i \in S} \pi_{S}^{i} x_{S}^{i}=\sum_{i \in S} \pi_{S}^{i} e^{i} \\
u^{i}\left(x_{S}^{i}\right) \geq g_{S}^{i}(v) \text { for all } i \in S .
\end{gathered}
$$

Furthermore, if $\lambda_{S}>0$, then $v$ is an element of $V^{c}(S)$ and therefore $g_{S}^{i}(v) \geq v^{i}$ for all $i \in S$. Define now the commodity allocation $\bar{x}_{N} \in X_{N}$ as follows. For each $i \in N$ let

$$
\bar{x}_{N}^{i}=\sum_{\substack{S \in \mathcal{N}: \lambda_{S}>0 \\ S \ni i}} n \lambda_{S} \pi_{S}^{i} x_{S}^{i}
$$

The consumption vector $\bar{x}_{N}^{i}$ is a weighted average of the vectors $x_{S}^{i}$ over those coalitions $S$ with positive $\lambda_{S}$ that contain player $i$. By convexity of the consumption sets, $\bar{x}_{N}^{i}$ is an element of $X^{i}$. By quasi-concavity of the utility functions,

$$
u^{i}\left(\bar{x}_{N}^{i}\right) \geq v^{i} \text { for all } i \in N
$$

Moreover,

$$
\begin{aligned}
\sum_{i \in N}\left[\bar{x}_{N}^{i}-e^{i}\right] & =\sum_{i \in N} \sum_{\substack{S \in N: \lambda_{S}>0 \\
S \ni i}} n \lambda_{S} \pi_{S}^{i}\left[x_{S}^{i}-e^{i}\right] \\
& =\sum_{S \in N_{i} \lambda_{S}>0} n \lambda_{S} \sum_{i \in S} \pi_{S}^{i}\left[x_{S}^{i}-e^{i}\right]=0 .
\end{aligned}
$$

We see that the allocation $\bar{x}_{N}$ is an element of the set $F(E)$. Since $v$ is not an interior point of any set $V^{\prime}(S)$, the allocation $\bar{x}_{N}$ is robust to deviations by fuzzy coalitions $\pi_{S} \in \Delta_{S}^{\prime}$ for all $S \in \mathcal{N}$. Thus, $\tilde{x}_{N}$ is in the $\epsilon$-fuzzy core of the economy $\mathscr{E}^{2}$. 
Theorem 3.4 The game $V^{c}$ is $\Psi$-balanced.

We omit the proof of this theorem as it is similar to the proof of Theorem 3.3. Theorem 3.4 implies that the $\Psi$-balanced core of the game $V^{k}$ is non-empty. Our argument is now complete. 


\section{Part II}

\section{Sequential Core Concepts}





\section{Chapter 4}

\section{The Strong Sequential Core in a Two-period Economy ${ }^{1}$}

\subsection{Introduction}

The fact that most economic interaction takes place over time has received limited attention on the part of cooperative game theory. Cooperative solution concepts like the core are traditionally defined in a static setup, where all decisions are made and carried out simultaneously. Such models provide a somewhat simplified picture of cooperative decision making. In a dynamic world the process of decision making is effectively separated from the process of implementing decisions, thus opening a possibility for a wide range of complex behavioral patterns not observed in a static setup. In Part II of the present monograph we study the implications of the unravelling of time and uncertainty for the concept of the core. Chapter 4 introduces the concept of the strong sequential core, which is also treated in Chapters 6 and 7 . Chapter 5 is devoted to the concept of the weak sequential core.

In Chapters 4 and 5 we focus on an exchange economy with two periods of time (zero and one) and with uncertainty as to the state of the economy in period one. The tracle in such an econony proceeds sequentially. In period zero the players trade in assets. An asset is a contract that specifies an amount of each commodity that the owner of the alsset is entitled to receive or is obliged to deliver, conditional on the state of the economy. In period one, the players learn the true state, and the delivery of commodities takes place, as specified by each player's portfolio of assets. After that, the players may engage in trade anew. This results in a final allocation of commodities, which is consumed.

When one considers the core of a static, one shot economy, there is no need to distinguish between binding and non-binding agreements. Once all players have approved of a certain redistribution of the initial endowments, exchange and consumption take place immediately, and nothing happens in between. This is radically different in a dynamic model. For example, in the two-period economy described above, two rounds of trade are separated by a release of information as to the state of an economy. This may lead the

\footnotetext{
${ }^{1}$ This chapter is based on Predtetchinski, Herings, and Peters [27].
} 
players to reconsider their decisions and create incentives to deviate from an agreement that has been partially carried out. The possibility to deviate from earlier agreements is an essential feature of cooperation in a dynamic environment. Therefore a distinction between enforceable and non-enforceable agreements becomes important in a dynamic setup.

One can think of the set of assets as representing all enforceable agreements. This means that no coalition of players can fail the delivery of goods as specified by the coalitional portfolio of assets. On the other hand, the agreements regarding the trade in commodities following the realization of the state of an economy are not enforceable. A player or a coalition can deviate from any such agreement, once the true state has been learned.

There are two types of coalitional deviations in a two-period exchange economy: ex ante coalitional deviations, i.e. those deviations that occur in period $t=0$, and ex post deviations that occur in period $t=1$ following the resolution of uncertainty. The classical core consists of those allocations that are robust to all ex ante coalitional deviations. In the classical core a coalition deviates from a proposal whenever there is a feasible redistribution of the total coalitional endowment of commodities that gives each member of the coalition a higher expected utility than the proposecl allocation.

If the payoffs of the assets span all of the commodity space, then the classical core appears to be an adequate solution concept. In this situation any classical core allocation can be achieved by an appropriate exchange of asset contracts, and thus enforced. Upon the realization of the state of an economy, asset contracts are executed, and no retrading of commodities is needed. Thus, with a complete set of assets, all necessary trade can be done in one round.

With an incomplete (e.g. empty) set of assets, not all allocations can be achieved after only one round of trade. Consequently, some of the allocations selected by the classical core may not be stable to coalitional deviations ex post, once the state of the economy is known. These considerations lead us to make a selection from the classical core. A pair consisting of an allocation of commodities and an allocation of assets is in the strong sequential core if the allocation of commodities is in the ex ante core, and if in each of the possible states it belongs to the core of the corresponding ex post subeconomy, where the executed asset contracts serve as initial endowments.

The strong sequential core increases with the number of assets. The more assets there are in the economy, the larger is the set of allocations that are robust to coalitional deviations in period one, the larger is the strong sequential core. If there is a strongly complete set of assets, then the strong sequential core coincides with the ex ante core.

As is evident from the definition, the strong sequential core is a very selective solution concept. It is therefore not very surprising that in many cases the strong sequential core is empty. A particularly sharp emptiness result can be obtained for finance economies, a special case of the general model where there is only one commodity in each state. If the difference between the number of states and the number of assets is equal to one, then in a generic finance economy the strong sequential core is a finite (possibly empty) set. If this difference is greater than one, then the strong sequential core is generically empty.

The organization of this chapter is as follows. Section 4.2 specifies the model and some preliminaries, and Section 4.3 defines the strong sequential core. Section 4.4 considers the 
special case of finance economies and Section 4.5 treats the case with multiple commodities. In Section 4.6 we define the concept of the strong* sequential core. The more involved proofs are collected in the Appendix.

\subsection{The model and preliminaries}

\section{The Model}

We consider an economy with two periods of time denoted by $t=0$ and $t=1$. In period $t=1$, the economy can be in one of the states $s=1, \ldots, s$, with the probability of state $s$ denoted by $\rho_{s}$. We assume that all states occur with positive probability: $\rho_{s}>0$ for all $s=1, \ldots, S$. Period $t=0$ is identified with state $s=0$.

There is a finite set $N$ of players. The cardinality of $N$ is denoted by $n$. There are $L$ commodities in each state of period $t=1$. An agent $i \in N$ is characterized by a consumption set $X_{s}^{i} \subset \mathbb{R}^{L}$, the vector of initial endowments $e_{s}^{i} \in X_{s}^{i}$, and the elementary utility function $u_{s}^{i}: X_{s}^{i} \rightarrow \mathbb{R}$ in the state of nature $s$. We use the notation $X^{i}=x_{s=1}^{S} X_{s}^{i}$ and $X=\times_{i \in N} X^{i}$. All players are assumed to be expected utility maximizers, with $v^{i}: X^{i} \rightarrow \mathbb{R}$ being the expected utility function defined by $v^{i}\left(x^{i}\right)=\sum_{s=1}^{S} \rho_{s} u_{s}^{i}\left(x_{s}^{i}\right)$.

Furthermore, there are $J$ assets in the economy, with the payoffs given by the $S L \times J$ dimensional matrix $A$. The generic entry $A_{s i}^{j}$ of the matrix $A$ specifies the quantity of commodity $l$ paid by asset $j$ in state $s$.

These components together define our economy, denoted by $\mathscr{E}$. We shall often parameterize economies by the players' endowments e. A typical element of this family is denoted by $\mathscr{E}_{e}$.

In period $t=0$, trade in assets takes place. There are no endowments and therefore no consumption in period $t=0$. In period $t=1$, nature randomly chooses the state of an economy. The delivery of goods takes place, as specified by each player's portfolio of assets. This results in an allocation of commodities denoted throughout by $x$. After that, the players may trade in commodities. Trade in commodities results in an allocation $y$, which is consumed.

Let $\langle A\rangle$ denotes the linear subspace of $\mathbb{R}^{S L_{S}}$ spanned by the columns of the matrix $A$. We define the set of constrained feasible allocations as

$$
\mathscr{A}=\left\{x \in \mathbb{R}^{n S L} \mid \sum_{i \in N} x^{i}=\sum_{i \in \mathbb{N}} e^{i}, x^{i}-e^{i} \in\langle A\rangle\right\} .
$$

Occasionally we shall write $\mathscr{A}_{c}$ rather than $\mathscr{A}$, to make a reference to an economy where the initial allocation is $e$. If there are no assets present in the economy, we define $\mathscr{A}$ to be a one-point set $\{e\}$.

The set $\mathscr{A}$ plays an important role in our analysis. It contains all allocations $x$ that may potentially arise as a result of trade in assets. Note that a constrained feasible allocation need not be an element of the consumption set $X$. Thus a player may have a debt at the 
beginning of period $t=1$, that is an allocation outside the consumption set. By the end of period $t=1$ all debts must be paid back, so that the final allocation $\bar{y}$ belongs to the consumption set.

\section{Subcanomies}

The ex ante economy, i.e. the one that precedes the resolution of uncertainty, may bet Associated with period $t=0$. This is the economy

$$
\mathscr{G}=\mathscr{G}\left(N, \mathbb{R}^{S L}:\left\langle X^{i}, v^{i}, e^{i}\right\rangle \in N\right)
$$

It has the commodity space $\mathbb{R}^{S L}$, the consumption sets $X^{i}$, the expected utility functions $y^{*}$, and the initial endowments $e^{i}$. An allocation $y \in X$ is ex ante Pareto efficient whenever it satisfes the following conditions:

(1) $\sum_{i \in N} y^{i}=\sum_{i \in N} e^{i}$, and

(2) there exists no allocation $z \in X$ such that $\sum_{i \in N} z^{i}=\sum_{i \in N} e^{i}$ and $v^{i}\left(z^{i}\right) \geq v^{i}\left(y^{i}\right)$ for all $z \in N$ with at least one strict inequality.

The set of all ex ante Pareto efficient allocations is denoted by $P\left(\mathscr{E}_{0}\right)$. The classical core of the econorny $\mathscr{G}_{0}$ is the set $C\left(\mathscr{G}_{0}\right)$ of allocations $y \in X$ satisfying the condition $(1)$ and

(3) there exist no coalition $M \subseteq N$, and no allocation $z \in x_{i \in M} X^{i}$ such that $\sum_{i \in M} z^{i}=$ $\sum_{\text {UEM }} e^{i}$ and $v^{i}\left(z^{i}\right)>w^{i}\left(y^{i}\right)$ for all $i \in M$.

In general, a classical core allocation only satisfies a weak version of Pareto efficiency and need not satisfy the condition (2) above. However, if for each player $i$ and every state $s$ the consumption set $X_{s}^{i}$ is given by the non-negative orthant $\mathbb{R}_{+}^{L}$ of the space $\mathbb{R}^{L}$, and the elementary utility function $u_{s}^{i}$ is continuous and strongly monotone ${ }^{2}$, then the wo versions of Pareto efficiency are equivalent and the inclusion $C\left(\mathscr{E}_{0}\right) \subseteq P\left(\mathscr{E}_{0}\right)$ does hold.

Any constrained feasible allocation $x$ gives rise to exactly $S$ ex post subeconomies, one in each of the possible states of nature, following the resolution of uncertainty. "The subconomy

$$
\mathscr{E}_{s i n}=\mathscr{E}\left(N, \mathbb{R}^{L},\left\langle X_{s}, u_{s}^{i}, x_{s}^{i}\right\rangle_{t \in N}\right)
$$

associated with state $s$ has commodity space $\mathbb{R}^{L}$, consumption sets $X_{s}^{i}$, utility functions $u_{s}^{i}$, and initial endowments $x_{s}^{i}$. The core of the subeconomy $\mathscr{E}_{s x}$ is the set $C\left(\mathscr{E}_{s x}\right)$ of allocations $y_{s} \in X_{s}$ sutiofying the following conditions:

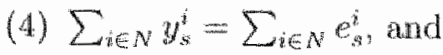

(5) there exist no coalition $M \subseteq N$, and no allocation $z_{s} \in X_{i \in M} X_{s}^{i}$ such that $\sum_{s \in M} z_{s}^{i}=$ $\sum$ in $x_{s}^{i}$ and $u_{s}^{i}\left(z_{s}^{i}\right)>w_{s}^{i}\left(y_{s}^{i}\right)$ for all $i \in M$.

The finction $u_{s}^{i}$ is strongly monotone if the following condition is satisfied: $\left[x_{s}^{i}, y_{s}^{i} \in X_{s}^{i}, x_{s !}^{i} \geq\right.$ $\left.y_{s i}^{i} \forall l \in\{1, \ldots, L\}, x_{s}^{i} \neq y_{s}^{i}\right]$ implies $\left[u_{s}^{i}\left(x_{s}^{i}\right)>w_{s}^{i}\left(y_{s}^{i}\right)\right]$. 
We remark that the core of the subeconomy $\mathscr{G}_{s x}$ is well-defined even if the constrained feasible allocation $x$ lies outside the consumption set $X$. In this case some coalitions may not be able to reject any proposal, since the set of allocations $z_{s}$ with which coalition $M$ can block in state $s$ may be an empty set. In particular, the conditions of individual rationality are valid only for those players $i$ whose vector $x_{s}^{i}$ is in the consumption set $X_{s}^{i}$. It should be stressed that the above definition does not involve the extension of the utility functions beyond the consumption sets.

Throughout the chapter we exploit the following example.

Example 4.1 Consider the family of economies, parameterized by the players' endowments e $\in X$, in which $N=\{a, b\}$ and both players have identical consumption sets and utility functions given by

$$
\begin{aligned}
X_{s}^{i} & =\mathbb{R}_{+++}^{L}, \\
u_{s}^{i}\left(y_{s}^{i}\right)=\sum_{i=1}^{L} \ln \left(y_{s l}^{i}\right), \quad y_{s}^{i} & \in \mathbb{R}_{++}^{L} .
\end{aligned}
$$

The probabilities of the states are $\rho_{s}=\frac{1}{S}$. Let the initial endowments $e \in X$ and the constrained feasible allocation $x \in \mathscr{A}_{e}$ be given.

The set of Pareto-efficient, allocations of the ex ante subeconomy $\mathscr{E}_{0}$ coincides with the diagonal of the Edgeworth box. That is, an allocation $y$ is ex ante Pareto efficient if and only if players' consumption bundles $y^{i}$ are proportional to the aggregate initial endowment, $e^{N}=e^{a}+e^{b}$ :

$$
P\left(\mathscr{E}_{0}^{a}\right)=\left\{\begin{array}{l|l}
\left(y^{a}, y^{b}\right) \in \mathbb{R}^{L S} \times \mathbb{R}^{L S} \mid \begin{array}{c}
\exists t^{a}, t^{b}>0: t^{a}+t^{b}=1 \\
y^{i}=t^{i} e^{N}, i=a, b
\end{array}
\end{array}\right\} .
$$

For an ex ante Pareto-efficient allocation to be in the ex ante core, the share of the aggregate endowment possessed by each player should be sufficiently large, so that the conditions of individual rationality hold. Thus,

$$
C\left(\mathscr{E}_{0}\right)=\left\{\left(y^{a}, y^{b}\right) \in \mathbb{R}^{L S} \times \mathbb{R}^{L S} \mid \begin{array}{c}
\exists t^{a}, t^{b}>0: t^{a}+t^{b}=1 \\
y^{i}=t^{i} e^{N}, i=a, b \\
t^{i} \geq \alpha_{0}^{i}(e), i=a, b
\end{array}\right\} .
$$

The value for $\alpha_{0}^{i}(e)$ is determined by the condition of individual rationality for player $i$. It. depends on the fundamentals of the economy as follows:

$$
\alpha_{0}^{i}(e)=\left(\prod_{s=1}^{S} \prod_{l=1}^{L} e_{s l}^{i} / e_{s l}^{N}\right)^{1 / S L}
$$

The core of the ex post subeconomy $\mathscr{E}_{s x}$ is given by

$$
C\left(\mathscr{E}_{s x}\right)=\left\{\begin{array}{l|l}
\left(y_{s}^{a}, y_{s}^{b}\right) \in \mathbb{R}^{L} \times \mathbb{R}^{L} \mid \begin{array}{c}
\exists t_{s}^{a}, t_{s}^{b}>0: t_{s}^{a}+t_{s}^{b_{s}}=1 \\
y_{s}^{i}=t_{s}^{i} e_{s}^{N}, i=a, b \\
t_{s}^{i} \geq \alpha_{s}^{i}(x), i=a, b
\end{array}
\end{array}\right\} .
$$


where

$$
\alpha_{s}^{i}(x)=\left\{\begin{array}{ll}
\left(\prod_{l=1}^{L} x_{s l}^{i} / e_{s i}^{N}\right)^{\mathbb{H} / L} & \text { if } x_{s}^{i} \in X_{s}^{i} \\
0 & \text { otherwise }
\end{array} .\right.
$$

Note that if the vector $x_{s}^{i}$ lise outside the consumption set $X_{s}^{i}$, the condition $t_{s}^{i} \geq \alpha_{*}^{i}(x)=0$ of individuat rationality for player in states is not binding.

\subsection{The strong sequential core}

Defnition 4.1 An allocation $\bar{y} \in X$ is an element of the strong sequential core SSC(X) of the economy of if $\bar{y} \in C\left(\mathscr{E}_{0}\right)$ and there exists an $\bar{x} \in \mathscr{A}$ such that $\bar{y}_{s} \in C\left(\mathscr{E}_{s}\right)$ for all $s=1, \ldots, s$.

Any allocation $\bar{y}$ in the strong sequential core is reached in two steps. In period $t=0$, players trade in assets. In period $t=1$, upon learning the true state of nature, the delivery of goods according to the players' portfolios of assets results in the intermediate allocation $\bar{x}$. After that trade in commodities takes place sund results in the allocation $\bar{y}$, which is consumed. The agreement to move from allocation $\vec{x}$ to allocation $\vec{y}$ is self-enforcing, since $y$ is in the core of the ax post sub economies corresponding to $\bar{x}$, in each state. The role of asset trachug is to change the initial allocation in period $t=1$ from $e$ to $\bar{x}$ so that, once $\vec{x}$ is reached, allocation $\bar{y}$ becomes robust against all ex post coditional deviations. Thus, in the strong sequential core, assets help to ensure ex post coalitional stability of the final allocation. The requirement that $\vec{y}$ be an element of the classical core ensures the ex ante coalitional stability of $\vec{y}$.

The more assets there are, the more allocations are robust against ex post coalitionat deviations. Thus the strong sequential core increases when the set of constrained feasible allocations expands. If the columns of the matrix $A$ span all of the space $\mathbb{R}^{S L}$, then the strong sequential cone contains the intersection of the classicall core and the set of ex ante Pareto efficient allocations. Indeed, any such allocation can be implemented directly by an appropriate trade in assets. Upon the realization of the state of nature, asset contracts are execubed, and no retrading of commodities is needed. Summarizing, we get the following theorem:

Theorem 4.1 If the set of assets traded in an economy expands, the strong sequential cone. weakly increases. If the matrix $A$ has rank $S L$, then $P\left(\mathscr{E}_{0}\right) \cap C\left(\mathscr{O}_{0}\right) \subseteq S S C(\mathscr{E}) \subseteq C\left(\mathscr{G}_{0}\right)$.

Proof. We prove the inclusion $P\left(\sigma_{0}\right) \cap C\left(\mathscr{G}_{0}\right) \subseteq S S C(\mathscr{\odot})$. Let $y$ be an ex ante Pareto efticient allocation in the classical core of the economy $\mathscr{E}_{0}$. If the matrix $A$ has rank $S L$, then the allocation $y$ is a constrained feasible allocation. We demonstrate that in each state $s$, the allocation $y_{s}$ is an element of the core of the economy $\&_{s y}$

Suppose not. Then there is a state $\sigma$, coalition $M$ and allocation $z_{\sigma} \in x_{4} X_{\sigma} X_{\sigma}$ such that $\sum_{i \in M} z_{\sigma}^{i}=\sum_{i \in M} f_{\sigma}^{i}$ and $u_{\sigma}^{i}\left(z_{\sigma}^{i}\right)>u_{\sigma}^{i}\left(y_{\sigma}^{i}\right)$ for all $i \in M$. Define $\bar{z} \in X$ by the equation

$$
\bar{z}_{s}^{i}= \begin{cases}z_{\sigma}^{i}, & \text { if } s=\sigma \text { and } \dot{x} \in M \\ y_{s}^{i}, & \text { otherwise. }\end{cases}
$$


Then the allocation $\ddot{z}$ is feasible for the grand coalition and $v^{i}\left(\bar{z}^{i}\right) \geq v^{i}\left(y^{i}\right)$ for all $i \in N$ with strict inequality for all $i \in M$. This contradicts the fact that $y$ is an ex ante Pareto efficient allocation. Therefore, $y_{s}$ belongs to the set $C\left(\mathscr{G}_{s y}\right)$ for all states $s$. Thus the allocation $y$ is an element of the strong sequential core.

If for each player $i$ and each state $s$ the consumption set $X_{s}^{i}$ is equal to $\mathbb{R}_{+}^{L}$, the elementary wtility function $u_{s}^{i}$ is strongly monotone and continuous, and the matrix $A$ has rank $S L$, then $\operatorname{SSC}(\mathscr{E})=C\left(\mathscr{E}_{0}\right)$.

\subsection{Finance economies}

We begin the analysis of the strong sequential core with the special case of finance economies. In a finance economy there is only one commodity in each state of nature; thus $L=1$.

Theorem 4.2 below relies on the following assumptions.

(A1) There are at least two players in the economy: $n \geq 2$.

(A2) The matrix $A$ of asset payoffs has full column rank $J$.

(A3) The consumption set $X^{i}$ is an open set of $\mathbb{R}^{s}$.

(A4) The function $v^{i}: X^{i} \rightarrow \mathbb{R}$ is twice continuously differentiable.

(A5) For any $x^{i} \in X^{i}, \partial v^{i}\left(x^{i}\right) \neq 0$.

(A6) If $x^{i} \in X^{i}, h \in \mathbb{R}^{S}, h \neq 0$, and $h^{\top} \partial v^{i}\left(x^{i}\right)=0$, then $h^{\top} \partial^{2} v^{i}\left(x^{i}\right) h<0$.

Theorem 4.2 Let $\mathscr{E}_{e}$ be a family of finance economies satisfying conditions (A1)-(A6) parameterized by the initial allocation $e \in X$. Then

1. If $J<S-1$, there exists a set of full measure $\mathscr{R} \subset X$ such that for all $e \in \mathscr{R}$ the set $P\left(\mathscr{E}_{0}\right) \cap \mathscr{A}_{e}$ is empty.

2. If $J=S-1$, there exists a set of full measure $\mathscr{R} \subset X$ such that for all $e \in \mathscr{R}$ the set $P\left(\mathscr{E}_{0 e}\right) \cap \mathscr{A}_{e}$ is discrete (possibly empty).

The proof of Theorem 4.2 can be found in the appendix. As the proof makes it clear, in Theorem 4.2 we do not have to assume that the functions $v^{i}$ have the expected utility form. The theorem is valid for any utility functions $v^{2}$ satisfying the above assumptions.

Now we retnun to our usual framework where the functions $w^{i}$ all have the expected utility form. Corollary 4.1 relies on the following set of assumptions.

(C1) For each $s=1, \ldots, S$ and each $i \in N$, the consumption set $X_{s}^{i}$ is the set of positive real numbers. 
(C2) For each $s=1, \ldots, S$ and each $i \in N$, the elementary utility function $u_{s}^{i}: X_{s} \rightarrow \mathbb{a}_{\mathrm{a}}$ is twice continuously differentiable.

(C3) For each $s=1, \ldots, S, i \in N$, and each $x_{s}^{i} \in X_{s}^{i}$, we have the inequalities $\partial u_{s}^{i}\left(x_{s}^{i}\right)>0$ and $\partial^{2} u_{*}^{i}\left(x_{y}^{2}\right)<0$.

(C4) For each $i \in N$, the set $\left\{y^{i} \in X^{i} \mid v^{i}\left(y^{i}\right) \geq v^{i}\left(e^{i}\right)\right\}$ of ex ante individually rational consumption bundles is closed in $\mathbb{R}^{S}$.

Observe that if the elementary utility functions $u^{i}$ satisfy conditions (C1)-(C4), then the corresponding expected utility functions $v^{i}$ satisfy conditions (A3)-(A6).

Corollary 4.1 Let $\mathscr{E}_{e}$ be a family of finance economies satisfying conditions (A1)-(A2) and (C1)-(CA) parameterized by the players's initial endowments $e \in X$. Let the subset $\mathscr{R}$ of $X$ be as in Theorem 4.2 .

1. If $J<S-1$, then for all $E \in \mathbb{R}$ the set $S S C\left(\mathscr{E}_{e}\right)$ is empty.

2. If $J=S-1$, then for all $E \in \mathscr{R}$ the set $S S C\left(\mathfrak{G}_{e}\right)$ is finite (possibly empty).

Proof. Let $x \in \infty$ be a constrained feasible allocation and $s$ be a state. First we show that the core of the subeconomy $\mathscr{B}_{s x}$ is given by the set

$$
C\left(\mathscr{E}_{s x}\right)=\left\{x_{s}\right\} \cap X_{s}
$$

That is, $C\left(\mathscr{E}_{s}\right)$ is a a one-point set $\left\{x_{s}\right\}$ whenever $x_{s} \in X_{s}$ and it is an empty set otherwise.

Suppose that $x_{s} \in X_{s}$. To see that $x_{s}$ is in the core of the subeconomy $\mathscr{E}_{s x}$, take any coalition $M$ and any allocation $z_{s} \in x_{i \in M} X_{s}^{i}$ such that $\sum_{i \in M} z_{s}^{i}=\sum_{i \in M} x_{s}^{i}$. Then $z_{s}^{i_{0}} \leq x_{s}^{i_{3}}$ for some member $i_{0}$ of coalition $M$. By monotonicity of the utility functions it must be that $u_{s}^{i_{0}}\left(z_{s}\right) \leq u_{s}^{i_{0}}\left(x_{s}^{i_{0}}\right)$. Thus, no coalition can improve upon allocation $x_{s}$. Conversely, let $y_{s}$ be in the core of the subeconomy $\mathscr{f}_{s . x}$. By individual rationality, we have $u_{s}^{i}\left(y_{s}^{i}\right) \geq u_{s}^{i}\left(x_{s}^{i}\right)$ implying that $y_{s}^{i} \geq x_{s}^{i}$ for all $i \in N$. Since both $y_{s}$ and $x_{s}$ are feasible for the grand coalition, wo have $y_{s}^{i}=x_{3}^{i}$ for all $i \in N$. Thus, $C\left(\mathbb{E}_{s x}\right)=\left\{x_{s}\right\}$.

Suppose that $x_{s}$ lies outside $X_{s}$. Then $x_{s}^{t_{0}} \leq 0$ for some $t_{0}$. Take any feasible allocation $y_{s} \in X_{s}$. We show that coalition $M=N \backslash\left\{i_{0}\right\}$ blocks $y_{s}$. Since $y_{s}^{i_{0}}$ is positive,

$$
\epsilon=\sum_{i \in M} x_{s}^{i}-\sum_{i \in M} y_{s}^{i}=-x_{s}^{i_{0}}+y_{s}^{i_{0}}
$$

is positive as well. Defne $z_{s} \in x_{i \in M} X_{s}$ by $z_{s}^{i}=y_{s}^{i}+\frac{1}{x_{2}}$ e. It is easy to see that $z_{s}$ is leasible for $M$ in the economy $\mathscr{c}_{s a}$ and is an improvement upon $y_{s}$. Thus, $C\left(\mathscr{E}_{s x}\right)$ is empty.

It follows that the strong sequential core of the economy $\mathscr{E}_{e}$ is given by the set

$$
\operatorname{SSC}\left(\mathscr{O}_{\mathbb{E}}\right)=C\left(\mathscr{G}_{0}\right) \bigcap \mathscr{A}_{\mathrm{e}}
$$

Under the maintained assumptions all allocations in the classical core of the econony $\mathscr{G}_{e}$ are ex ante Pareto efficient. The strong sequential core is therefore contained in the set 
$P\left(\mathscr{C}_{0}\right) \cap \mathscr{A}_{\mathrm{e}}$. The first part of the corollary then follows immediately from Theorem 4.2. For the second part, it suffices to show that $S S C(\mathscr{E})$ is a compact set.

It follows from assumption (C4) that the set of ex ante individually rational constrained feasible allocations

$$
\left\{y \in X \mid v^{i}\left(y^{i}\right) \geq v^{i}\left(e^{i}\right) \text { for all } i \in N\right\} \bigcap \mathscr{A}
$$

is a compact subset of $\mathbb{R}^{S N}$. It remains to show that the classical core of $\mathscr{G}_{0}$ is closed in $\mathbb{R}^{S N}$.

Consider the sequence $y_{q}$ in $C\left(\mathscr{E}_{0}\right)$ converging to a point $y_{0} \in \mathbb{R}^{S N}$. Suppose that there is a coalition $M \subseteq N$ and an allocation $z$, feasible for $M$, such that $v^{i}\left(z^{i}\right)>v^{i}\left(y_{0}^{i}\right)$ for all $i \in M$. Then, by continuity of $v^{i}$, for $q$ large enough the inequalities $v^{i}\left(z^{i}\right)>v^{i}\left(y_{i}^{i}\right)$ hold true, implying that $y_{q}$ is not an element of $C\left(\mathscr{G}_{0}\right)$, a contradiction. Thus, $y_{0}$ is in $C\left(\mathscr{E}_{0}\right)$.

The example below complements the second part of Corollary 4.1 . We show that, when $J=S-1$, then there may exist two non-empty disjoint open subsets of $X$, such that for alli initial allocations in the first set the strong sequential core is a finite non-empty set, and for all those in the second set the strong sequential core is empty.

Example 4.2 Consider the family of the economies of Example 4.1. Let $S \geq 3, L=1$, $J=S-1$, and let the $(S \times J)$-dimensional matrix of asset payoffs be given by

$$
A=\left(\begin{array}{cccc}
1 & 0 & \cdots & 0 \\
0 & 1 & \cdots & 0 \\
\vdots & \vdots & \ddots & \vdots \\
0 & 0 & \cdots & 1 \\
0 & 0 & \cdots & 0
\end{array}\right) .
$$

Then the set of ex ante Pareto efficient allocations intersects the set of constrained feasible allocation in a one-point set

$$
P\left(\mathscr{C}_{0 e}\right) \cap \mathscr{A}=\left\{\left(t^{\alpha}(e) e^{N}, t^{b}(e) e^{N}\right)\right\},
$$

where $t^{i}(e)=e_{S}^{i} / e_{S}^{N}$ is the fraction of the total endowment in state $S$ owned by agent $i$. It follows that the strong sequential core is either an empty set or a one point seli. It is a one-point set if the allocation defined by Equation 4.6 is individually rational, and it is empty otherwise. The former is the case if $t^{i}(e) \geq \alpha_{0}^{i}(e)$ for both players $a$ and $b$.

One can easily check that for a sufficiently small positive number $\epsilon$, the initial allocation

$$
\left(\begin{array}{c}
e^{a} \\
\bar{e}^{b}
\end{array}\right)=\left(\begin{array}{cccccc}
2-\epsilon & \epsilon & 1 & \ldots & 1 & 1 \\
\epsilon & 2-\epsilon & 1 & \ldots & 1 & 1
\end{array}\right)
$$

satisfies the inequalities $t^{i}(\bar{e})>\alpha_{0}^{i}(\bar{e})$ for $i=a, b$. Thus, the allocation $\left(\bar{e}^{a}, \bar{e}^{b}\right)$ is the interior point of the set of the initial allocations for which the strong sequential core is a one point set given by Equation 4.6. For any number $0<\epsilon<1$, the initial allocation

$$
\left(\begin{array}{l}
\bar{e}^{1} \\
\bar{e}^{2}
\end{array}\right)=\left(\begin{array}{cccccc}
1 & \mathbb{1} & 1 & \ldots & 1 & \epsilon \\
1 & \mathbb{1} & 1 & \ldots & 1 & 2-\epsilon
\end{array}\right)
$$


satisfies the inequality $t^{a(}(\bar{e})<\alpha_{0}^{a}(\bar{e})$. Therefore, it belongs to the interior of the set of those mitial allocations for which the strong sequential core is empty.

\subsection{The multiple commodities case}

In this section we consider the economies where there are at least two goods in each state of nature.

We have already noted that the strong sequential core coincides with the classical core if the matrix $A$ has rank $S L$, and the utility functions are continuous and strongly monotone. Strong completeness of the set of assets is a weaker condition which is still sufficient for the coincidence of the classical core and the strong sequential core.

Definition 4.2 We say that there is a strongly complete set of assets if for every vector $p \in \mathbb{R}_{++}^{S L}$ the $(S \times J)$-dimensional matrix

$$
\left(\begin{array}{ccc}
p_{1} A_{1}^{1} & \cdots & p_{1} A_{1}^{J} \\
\vdots & & \vdots \\
p_{S} A_{S}^{1} & \cdots & p_{S} A_{S}^{J}
\end{array}\right)
$$

with a generic entry $p_{s} A_{s}^{j}=\sum_{l=1}^{L} p_{s l} A_{s l}^{j}$ has rank $S$.

If the matrix $A$ has $S L$ linearly independent columns, then the set of assets is strongly complete.

Theorem 4.3 Let $\mathscr{E}$ be an economy where for each player $i$ and for each state s the consumption set $X_{s}^{i}$ coincides with $\mathbb{R}_{+}^{L}$, the elementary utility function $u_{s}^{i}$ is continuous, concave, and strongly monotone, and the set

$$
\left\{y^{i} \in X^{i} \mid v^{i}\left(y^{i}\right) \geq v^{i}\left(e^{i}\right)\right\}
$$

of ex ante indundually rationah consumption burdles is entirely contained in the interior of $X^{\prime}$. Suppose that there is a strongly complete set of assets. Then $\operatorname{SSC}(\mathscr{G})=C\left(\mathscr{G}_{0}\right)$.

The proof of Theorem 4.3 can be found in the appendix.

As Example 4.4 below shows, apart from the case where a strongly complete set of assets is available for trade, the strong sequential core can be empty. In contrast, Example 4.3 shows that even without any assets, an economy can have a non-empty strong sequential core. Both examples are robust to small perturbations of the initial endowments. In particular, Example 4.3 implies that Corollary 4.1 cannot be extended to economies with multiple conmodities. 
Example 4.3 Consider a family of the economies $\mathscr{E}_{e}$ as in Example 4.1. Suppose that $L>1$ and that there are no assets; thus $J=0$. The strong sequential core of the economy $\mathscr{E}_{e}$ is given by the set

$$
\operatorname{SSC}\left(\mathscr{G}_{e}\right)=\left\{\begin{array}{l|l}
\left(y^{a}, y^{b}\right) \in \mathbb{R}^{L S} \times \mathbb{R}^{L S} \mid \begin{array}{c}
\exists t^{a}, t^{b}>0: t^{a}+t^{b}=1 \\
y^{i}=t^{i} e^{N} \\
t^{i} \geq \alpha_{0}^{i}(e), t^{i} \geq \alpha_{s}^{i}(e) \\
i=a, b, s=1, \ldots, S
\end{array}
\end{array}\right\} .
$$

It is non-empty if and only if for all states $s$ and $\sigma$ the inequalities

$$
\alpha_{s}^{a}(e)+\alpha_{\sigma}^{b}(e) \leq 1
$$

hold. One can easily see that for any sufficiently small positive $\epsilon$ the state-independent initial allocation

$$
\left(\begin{array}{l}
e_{s}^{a} \\
\bar{e}_{s}^{b}
\end{array}\right)=\left(\begin{array}{ccccc}
\epsilon & 2-\epsilon & 1 & \ldots & 1 \\
2-\epsilon & \epsilon & 1 & \ldots & 1
\end{array}\right), s=1_{y} \ldots, S
$$

satisfies the strict inequalities $\alpha_{s}^{a}(\vec{e})+\alpha_{\sigma}^{b}(\vec{e})<1$ for all $s$ and $\sigma$. Thus the allocation $\vec{e}$ belongs to the interior of the set of those initial allocations for which the strong sequential core is non-empty. By Theorem 4.1, we can augment the economy by any number of assets while preserving the robust non-emptiness of the strong sequential core.

Example 4.4 Consider a family of the economies $\mathscr{E}_{\mathrm{e}}$ as in Example 4.1. Suppose that $L>1$ and $A_{S l}^{j}=0$ for all $l=1, \ldots, L$, and all $j=1, \ldots, J$. This excludes the case of a strongly complete set of assets. The asset payoffs in states other than state $S$ can be chosen arbitrarily.

The strong sequential core of the economy $\mathscr{E}_{\mathrm{e}}$ is given by the set

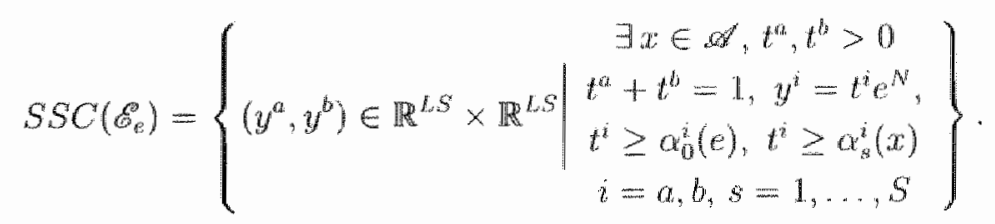

It is empty whenever the initial allocation e satisfies the inequality

$$
\alpha_{S}^{a t}(e)+\alpha_{0}^{b}(e)>1
$$

Indeed, take any such allocation $e$. Let $x \in \mathscr{A}_{e}$ be a constrained feasible allocation. Since no asset pays off in state $S$, we see that $x_{S}^{a}=e_{S}^{a}$, so $\alpha_{S}^{a}(e)=\alpha_{S}^{a}(x)$, and therefore $\alpha_{S}^{a}(x)+\alpha_{0}^{b}(e)>1$. This inequality means that the condition of individual rationality for player $a$ in state $S$ is incompatible with that for player $b$ in period $t=0$. Because $x$ was chosen arbitrarily in $\mathscr{A}_{e}$, the strong sequential core of the economy $\mathscr{E}_{\mathrm{e}}$ is empty. 


\subsection{The strong* sequential core}

In this section we explore a modification of Definition 4.1 where one requires that a constrained feasible allocation $\bar{x}$ be an element of the consumption set $X$. The modified solution concept is called the strong" sequential core.

Definition 4.3 An allocation $\bar{y} \in X$ is an element of the strong sequential core $\operatorname{SSC}^{*}\left(\mathscr{E}^{*}\right)$ of the economy $\mathscr{E}$ if $\bar{y} \in C\left(\mathscr{G}_{0}^{*}\right)$ and there exists an $\bar{x} \in \mathscr{A} \cap X$ such that $\bar{y}_{s} \in C\left(\mathscr{G}_{s \bar{x}}\right)$ for all $s=1, \ldots, S$.

One can verify that Theorem 4.1 remains true for a modified version of the strong sequential core. Corollary 4.1 holds, because $S S C^{*}(\mathscr{E}) \subseteq S S C(\mathscr{E})$. As Example 4.5 makes it clear, Theorem 4.3 is no longer true when we consider the strong* sequential core. It turns out that the strong ${ }^{*}$ sequential core may be empty, even when there is strongly complete set of assets in the economy.

Example 4.5 Consider an economy $\mathscr{E}$ as in Example 4.1, where there are two states, two commodities, and two assets: $S=2, L=2$, and $J=2$. 'The players' initial endowments and the payoffs of the assets are specified in the table below.

$$
\begin{array}{cccccc} 
& & e_{s l}^{a} & e_{s l}^{b} & A_{s l}^{1} & A_{s l}^{2} \\
s=1 & l=1 & 1 & 4 & 1 & 0 \\
s=1 & l=2 & 1 & 4 & 0 & 1 \\
s=2 & l=1 & 4 & 1 & 0 & 0 \\
s=2 & l=2 & 4 & 1 & 0 & 1
\end{array}
$$

The set of assets of the economy $\mathscr{E}$ is strongly complete, since for any vector $p=\left(p_{11}, p_{12}\right.$, $\left.p_{21}, p_{22}\right) \in \mathbb{R}_{++}^{S L_{-}}$the matrix

$$
\left(\begin{array}{cc}
p_{11} & p_{12} \\
0 & p_{22}
\end{array}\right)
$$

has full rank $S=2$.

Claim 4.1 The strong* sequential core of the economy $\mathscr{E}$ is empty.

The strong* sequential core of the economy $\mathscr{E}_{e}$ is the set

$$
S S C^{*}(\varepsilon)=\left\{\begin{array}{ll} 
& \exists x \in \mathscr{A} \cap X, t^{t a}, t^{b}>0 \\
\left(y^{a}, y^{b}\right) \in \mathbb{R}^{L S} \times \mathbb{R}^{L S} \mid \begin{array}{c}
t^{a}+t^{b}=1, y^{i}=t^{i} e^{N} \\
t^{i} \geq \alpha_{0}^{i}(e), t^{i} \geq \alpha_{s}^{i}(x) \\
i=a, b, s=1,2
\end{array}
\end{array}\right\} .
$$

Let $x \in \mathscr{A} \cap \mathrm{X}$ be a constrained feasible allocation. Letting $\theta$ be player $a$ 's net trade in asset $j=2$, we have the following equations:

$$
\begin{aligned}
& x_{12}^{a}=e_{12}^{a}+\theta=1+\theta \\
& x_{211}^{a+1}=e_{21}^{a}=4 \\
& x_{22}^{a}=e_{22}^{a}+\theta=4+\theta .
\end{aligned}
$$


Since $x^{a}$ is in the consumption set of player $a_{4}$ we have $x_{12}^{a x}>0$. It follows that $\theta>-1$, and that $x_{22}^{a}>3$. Therefore,

$$
\alpha_{2}^{a}(x)=\left(\frac{x_{21}^{a}}{e_{21}^{N}} \cdot \frac{x_{22}^{a}}{e_{22}^{N}}\right)^{1 / 2}>\left(\frac{4}{5} \cdot \frac{3}{5}\right)^{1 / 2} \approx 0.69 .
$$

On the other hand, $\alpha_{0}^{b}(e)=0.4$. Whence the inequality

$$
\alpha_{2}^{a}(x)+\alpha_{0}^{b}(e)>1 .
$$

Thus the condition of ex post indiwidual rationality for player $a$ in state $s=2$ is not compatible with the condition of ex ante individual rationality for player $b$. Because $x$ was chosen arbitrarily in $\mathscr{A} \cap X$, the strong* sequential core of the economy $\mathscr{E}$ is empty.

A transfer of commodities from agent $a$ to agent $b$ in the state of nature $s=2$ might solve the problem. It could diminish the value of $\alpha_{S}^{a}$ and thus weaken the condition of individual rationality for agent $a$ in the state of nature $s=2$. However, the only asset that pays in state $s=2$ is asset $j=2$ and it also pays in state $s=1$. Given that the initial endowment $e_{12}^{a}$ is sufficiently small, any attempt to redistribute a unit of asset $j=2$ from player $a$ to player $b$ results in an allocation $x$ that prescribes to player $a$ a negative amount $x_{12}^{m}$ of commodity $l=2$ in state $s=1$. However, such an allocation is prohibited by the definition of the strong* sequential core.

\section{Appendix}

\section{A.1 Proof of Theorem 4.2}

Define a subset of $X \times X$ as follows. Let a point $(e, x) \in X \times X$ be in $\mathscr{H}$ whenever $x$ is an ex ante Pareto-efficient constrained feasible allocation of the economy $\mathscr{b}_{s:}$. That is,

$$
\mathscr{M}=\left\{(e, x) \in X \times X \mid x \in P\left(\mathscr{E}_{0}\right) \cap \mathscr{A}\right\}
$$

As a first step of the proof, we argue that $A$ is an $((n-1)(J+1)+S)$-dimemsional $C^{1}$-submanifold of $X \times X$.

Let the set $\mathscr{S}$ be defined by

$$
\mathscr{P}=\left\{(e, \theta) \in X \times \mathbb{R}^{n J} \mid e^{i}+A \theta^{i} \in X^{n}, \sum_{i \in \mathbb{N}} \theta^{i}=0\right\} .
$$

Then $\mathscr{P}$ is a smooth $(S n+J n-J)$-dimensional manifold. Let $\mathscr{S}^{S-1}$ be $(S-1)$-dimensional unit sphere, and $\left(5^{S^{5}-1}\right)^{n}$ be the product of $n$ such spheres. Let $\Delta$ be a diagonal of $\left(\mathscr{P}^{s-1}\right)^{n}$, i.e. a set given by

$$
\Delta=\left\{\left(z^{1}, \ldots, z^{n}\right) \in\left(\mathscr{S}^{S-1}\right)^{n_{1}} \mid z^{1}=\cdots=z^{n}\right\}
$$


Then $\Delta$ is a smooth $(S-1)$-dimensional submanifold of $\left(S^{S-1}\right)^{n}$. Define a $C^{1}-$ function $g^{i}: X^{i}-\operatorname{Pos}^{s-1}$ by letting

$$
g^{i}\left(y^{i}\right)=\frac{\partial v^{i}\left(y^{i}\right)}{\left\|\partial v^{i}\left(y^{i}\right)\right\|}
$$

be a nomalized gradient of the function $v^{i}$, for each $y^{i} \in X^{2}$. Let a $C^{1}$ - function $h: 9^{\circ} \rightarrow$ $\left(\operatorname{ses}^{s-1}\right)^{\text {th }}$ be defined by

$$
h(e, \theta)=x_{i \in N g^{i}}\left(e^{i}+A \theta^{i}\right)
$$

for all $(e, \theta) \in Q$

Under the assumptions of Theorem 4.2 , the function $g^{i}$ is a submersion: at each point of $X^{i}$ the differential of $g^{i}$ is a surjective linear map, sec Debreu [9]. Differentiating the function $h$ with respect to $e$, one can easily see that $h$ is also a submersion. It follows that $h$ is transverse to any submanifold of the manifold $\left(5^{S-1}\right)^{\mathrm{n}}$. In particular, it is transverse to $\Delta$. It follows that the preimage of $\Delta$ under $h$ is an $((n-1)(J+1)+S)$-dimensional

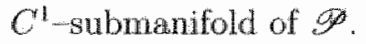

It is very well-known that a feasible allocation $x$ is Pareto-efficient if and only if the normalized gradients $g^{i}$ at $x^{i}$ conncide across all players. Consequently, the set $\mathscr{A}$ can be easily shown to be smoothly diffeomorphic to the subset of $\mathscr{P}$ given by the preimage of $\Delta$ under the function $h$. Thus $\mathscr{H}$ is an $((n-1)(J+1)+S)$-dimensional $C^{1}$-submanifold of $X \times X$.

Let $\pi: \mathscr{A} \rightarrow X$ be a natural projection. Then $\pi$ is a $C^{\mathrm{l}}$ map between two $C^{\mathrm{i}}-$ manifolds. Let $\mathscr{R}$ denote the set of regular values of the function $\pi$. By the theorem of Sard, the complement of $\mathscr{R}$ in $X$ has Lebesgue measure zero.

If $J<S-1$, then the dimension of the manifold $\mathscr{A}$ is smaller than that of $X$. Therefore, a point $e \in X$ is a regular value of the function $\pi$ only if it is outside the image of $\pi$. Thus, $\pi^{-1}(e)$ is an empty set for each $e \in \mathscr{R}$. This proves the first part of Theorem 4.2.

If $J=S-1$, then the manifolds $\mathscr{A}$ and $X$ both have dimension $n S$. In this case, $\pi^{-1}(e)$ is a zeromdimensional manifold for each $e \in \mathscr{R}$. Observe that any manifold of dimension zero is a discrete set. This proves the second part of the theorem.

\section{A.2 Proof of Theorem 4.3}

As a first step, we show that the ex ante Pareto-efficiency implies ex post Pareto-ficiency: if $y$ is a Pareto efficient allocation of the ex ante subeconomy $\sigma_{0}^{*}$, then $y_{s}$ is a Pareto efficient allocation of the ex post subeconomy $\mathscr{G}_{\text {se }}$ for each state $s$.

Suppose not. Then for some state $\sigma$ the allocation $y_{0}$ is not Pareto-ficient in the subeconomy $\mathscr{E}_{\sigma e}$. In this case there exists a feasible allocation $z_{\sigma} \in X_{\sigma}$ such that $u_{\sigma}^{i}\left(z_{\sigma}^{i}\right) \geq$ $u_{o}^{i}\left(y_{\sigma}^{i}\right)$ for all $z \in N$, where at least one inequality is strict. Define the allocation $\bar{z} \in X$ as follows:

$$
\vec{z}_{s}= \begin{cases}z_{\sigma}^{i} & \text { if } s=\sigma \\ y_{s}^{i} & \text { otherwise. }\end{cases}
$$


Then $z$ is feasible and $v^{i}\left(\bar{z}^{i}\right) \geq v^{i}\left(y^{i}\right)$ for all $i \in N$ with at least one strict inequality. This contradicts the ex ante Pareto efficiency of allocation $y$. Thus $y$ must be Pareto-efficient in each of the ex post subeconomies $\mathscr{E}_{s e}$.

Let $\bar{y}$ be in the classical core of the economy $\mathscr{E}_{0}$. We must find a constrained feasible allocation $\bar{x}$ such that $\bar{y}_{s}$ is contained in the core of the ex post subeconomy $\mathscr{g}_{s, \bar{x}}$, for all $s=1, \ldots, S$.

Under the assumptions of the theorem, the inclusion $C\left(\mathscr{E}_{0}\right) \subseteq P\left(\mathscr{f}_{0}\right)$ holds. Thus the allocation $\bar{y}$ is ex ante and therefore ex post Pareto efficient. By the Second Welfare theorem (see Mas-Colell, Whinston, and Green [22, Proposition 16.D.1]) there exist nonzero vectors of commodity prices $p_{s} \in \mathbb{R}^{L}$ such that the tuple $\left(\bar{y}_{s}, p_{s}\right)$ is a quasiequilibrium with transfers of the ex post subeconomy $\mathscr{E}_{s e}$. Since $\bar{y}^{i}$ is ex ante individually rational, it belongs to the interior of the consumption set $X^{i}=\mathbb{R}_{+}^{S L}$. This fact together with the strong monotonicity of $u_{s}^{i}$ ensure that the pair $\left(\tilde{y}_{s}, p_{s}\right)$ is an equilibrium with transfers and that the price vector $p_{s}$ is strictly positive.

By the assumption of a strong completeness of the set of assets, the system of equations

$$
\left(\begin{array}{c}
w_{1}^{i} \\
\vdots \\
w_{S}^{i}
\end{array}\right)=\left(\begin{array}{c}
p_{1} e_{1} \\
\vdots \\
p_{S} e_{S}
\end{array}\right)+\left(\begin{array}{ccc}
p_{1} A_{1}^{1} & \cdots & p_{1} A_{1}^{j} \\
\vdots & & \vdots \\
p_{S} A_{S}^{1} & \cdots & p_{1} A_{1}^{j}
\end{array}\right) \theta^{i}
$$

has a solution with respect to $\theta^{i}$ for all $i \in N$. Denote this solution by $\ddot{\theta}^{i}$ and define the constrained fessible allocation $\bar{x}$ by $\bar{x}^{i}=e^{i}+A \bar{\theta}^{i}$ for all $i \in N$. Since $p_{s} \bar{x}_{s}^{i}=w_{s}^{i}$ for all $i \in N,\left(\bar{y}_{s}, p_{s}\right)$ is a Walrasian equilibrium of the ex post subeconomy $\mathscr{E}_{s \bar{x}}$ in state $s$. It follows that $\bar{y}_{s}$ is in the core of the economy $\mathscr{E}_{s \bar{x}}$, as desired. 



\section{Chapter 5}

\section{The Weak Sequential Core in a Two-period Economy ${ }^{1}$}

\section{$5.1 \quad$ Introduction}

The analysis in Chapter 4 was motivated by the fact that the classical core allocations may not be self-enforcing once the state of an economy is known. The concept of the strong sequential core takes this consideration into account by selecting only those classical core allocations that belong to the core of an ex-post subeconomy in each state, given an appropriate allocation of asset contracts.

One may argue, however, that the definition of the strong sequential core is not self consistent in the following sense: while it is required that the final allocation be stable once the state has been revealed, no similar stability requirement is imposed upon the deviations of coalitions. It may be the case that the deviation by a coalition that promised improvement in ex ante terms, can in turn be improved upon by some sub-coalition once the true state of the economy is known. In this case, it could be argued that the coalitional deviation at hand is not "credible" since it may be counterblocked by some subcoalition. The concept of the weak sequential core abandons the condition that the final allocation be in the classical core. A weaker condition is imposed, namely that the final allocation should be robust solely against "credible" deviations.

For static cooperative situations, it is known that the restriction to credible deviations by coalitions does not alter the definition of the core: an allocation can be blocked by some coalitional deviation if and only if it can be blocked by a credible coalitional deviation (see Ray [28]). Kranich, Perea and Peters [20] show, however, that, this restriction becomes relevant if one turns to dymamic cooperative environments. As will become clear in this chapter, the focus on credible deviations is crucial as well for our context of two-stage economies. Consequently, the weak sequential core for such economies is in general a strict superset of the strong sequential core.

The concept of the Two-Stage core, due to Koutsongeras [17], is obtained under the

\footnotetext{
This chapter is based on Predtetchinski, Herings, and Perea [25].
} 
assumption that if a deviation occurs before the true state of nature is known, no exchange can take place within the deviating coalition once the state has been revealed. Whenever a number of standard conditions is satisfied, the weak sequential core is a subset of the two-stage core.

We show that the weak sequential core is non-empty in a number of important special cases. One instance is the finance economy, where the weak sequential core concides with the two-stage core. Whenever a complete set of state-contingent contracts is available for trade, co-existence of the weak sequential core, strong sequential core, and the two-stage core holds. Finally, we prove that the weak sequential core is non-empty for economies with two agents.

We give an example of an economy with three agents and no assets where the weak sequential core is empty. As we discussed in Chapter 4, the strong sequential core is weakly increasing in the number of assets. This result does not carry over to the weak sequential core. In fact, we demonstrate that the weak sequential core may be non-empty in an economy with no assets, while becoming empty after introducing a single asset in this econorny.

The outline of the chapter is as follows. In Section 5.2 we introduce the necessary notation. In Section 5.3 the concept of the weak sequential core is introduced and is compared to the concepts of the strong sequential core and the two-stage core. Some special cases are considered in Section 5.4. Section 5.5 introduces an NTU-game associated with the two period economy. Section 5.6 contains examples.

\subsection{Constrained feasible allocations and ex post sube- conomies}

We consider a two-period economy as presented in Section 4.2. Given a coalition $M \subseteq N$, define the set of constrained $M$-feasible allocations by

$$
\mathscr{S \mathbb { P } ^ { * }}(M)=\left\{x \in \underset{i \in M}{\times} X^{i} \mid \sum_{i \in M} x^{i}=\sum_{i \in M} e^{i}, x^{i}-e^{i} \in\langle A\rangle\right\} .
$$

The above definition requires a constrained efficient allocation to be in the consumption sets. This requirement not only simplifies our analysis, but it is essential for some of the results of this chapter (Theorem 5.4).

Given a constrained $M$ feasible allocation $x \in \mathscr{A}^{*}(M)$ we define the ex post subeconomy

$$
\mathscr{E}_{s}(x, M)=\mathscr{E}\left(M, \mathbb{R}^{L},\left(X_{s}^{i}, u_{s}^{i}, x_{s}^{i}\right\rangle \text { ieM }\right) \text {. }
$$

in state $s$ as a pure exchange economy with a set of agents $M$, commodity space $\mathbb{R}^{L}$, consumption sets $X_{s}^{i}$, utility functions $u_{s}^{i}$, and initial endowments $x_{s}^{i}$. The classical core $C\left(\mathscr{E}_{s}(x, M)\right)$ of the econony $\mathscr{E}_{s}(x, M)$ is the set of allocations $y \in x_{i \in M} X^{i}$ satisfying the following conditions: 
(1) $\sum_{i \in M} y_{s}^{i}=\sum_{i \in M} x_{s}^{i}$, and

(2) there exisi; no coalition $Q \subseteq M$, and no allocation $z_{s} \in X_{i \in Q} X_{s}^{i}$ such that $\sum_{i \in Q} z_{s}^{i}=$ $\sum_{i \in Q} x_{s}^{i}$ and $u_{s}^{i}\left(z_{s}^{i}\right)>u_{s}^{i}\left(y_{s}^{i}\right)$ for all $i \in Q$.

\subsection{Three concepts of the sequential core}

In this section we compare the concept of the weak sequential core to that of the strong sequential core and of the two-stage core. We start with the definition of the werk sequential core.

Definition 5.1. An allocation $\bar{y}$ is an element of the weak sequential core $W S C(\mathscr{E})$ of the economy $\mathscr{E}$ if

1. there exists an $\bar{x} \in \mathscr{O}^{*}(N)$ such that $\bar{y}_{s} \in C\left(\mathscr{E}_{s}(\bar{x}, N)\right)$ for all $s$, and

2. there exist no coalition $M \subseteq N$ and no allocations $y \in x_{i \in M} X^{i}$ and $x \in \mathscr{A}{ }^{*}(M)$ such that $y_{s} \in C\left(\mathscr{E}_{s}(x, M)\right)$ for all $s$ and $v^{i}\left(y^{i}\right)>v^{i}\left(\bar{y}^{i}\right)$ for all $i \in M$.

Part one of the definition is the requirement that the allocation $\bar{y}$ be robust against the deviations that can potentially take place at period one, after the true state of nature has been revealed. Part two of the definition is the requirement that the allocation $\bar{y}$ be robust against all credible deviations at period zero, before the true state of nature is known.

The three concepts of the sequential core - weak sequential core, strong sequential core, and two stage core - agree on the meaning of the improvement by a coalition at period $t=1$. Since agents face no uncertainty and no prospects of future consumption at period one, the classicall definition of an improving coalition seems to be appropriate, with the initial positions of coalitions being determined by the allocation $x$. This resultis in the requirement that $\bar{y}_{s}$ be in the classical core of the conomy $\mathscr{E}_{s}\left(\tilde{x}_{s} N\right)$, for all the states $s$.

The situation differs as we turn to period zero. It is not obvious which cleviations should be taken into account, and which not. Whence, a discrepancy between the three concepts of the sequential core as to the definition of an improvement by a coalition for period zero. The idea in the weak sequential core is to focus on credible deviations.

Suppose that a coalition $M$ considers to deviate at period zero to allocation $y$, which is preferred, in expected terms, to allocation $\bar{y}$ by all the members of $M$. In general, there may be counter-deviations from $y$ by sub-coalitions of $M$ once the true state of nature is known. Coalition $M$, however, might redistribute assets among its members at period zero in such a way that, given this redistribution, no deviations from $y$ will be profitable in the subsequent period. If there does exist such a redistribution of assets within the coalition $M$, then $y$ can be regarded as a credible deviation. Otherwise it is not credible, for there may be counter-deviations from $y$ at period one. The weak sequential core requires its elements to be robust only against credible deviations, rather than against all possible deviations in the classical sense. 
Definition 5.2 An allocation $\bar{y}$ is an element of the strong* sequential core $S S C^{*}\left(\mathscr{C}^{\circ}\right)$ of the economy $\mathscr{E}^{6}$ if

1. there exists an $\bar{x} \in \mathscr{Z}^{*}(N)$ such that $\vec{y}_{s} \in C\left(\mathscr{E}_{s}(\bar{x}, N)\right)$ for all $s$, and

2. there exist no coalition $M \subseteq N$ and no allocation $y \in x_{i \in M} X^{i}$ such that $\sum_{i \in M} y^{i}=$ $\sum_{i \in M} e^{i}$ and $v^{i}\left(y^{i}\right)>v^{i}\left(\bar{y}^{i}\right)$ for all $i \in M$.

The difference between Definition 5.1 and Definition 5.2 is that the latter does not require deviations of coalitions at period zero to be robust to possible counter-deviations by subcoalitions in period one. Instead, the classical notion of improvement is adopted: a coalition improves upon the allocation $\tilde{y}$ by the allocation $y$, if the allocation $y$ is feasible and gives each nember of the deviating coalition more expected utility than the allocation $\bar{y}$ does. Any element of the strong* sequential core must therefore be stable against all the deviations in the classical sense, whether credible or not. Thus, the strong* sequential core is a subset of the weak sequential core.

We proceed with the definition of the weak sequential core, due to Kontsougeras [17].

Definition 5.3 An allocation $\bar{y}$ is an element of the two-stage core of the economy $\mathcal{E}$, denoted $\operatorname{TSC}(\mathscr{E})$, if

1. there exists an $\ddot{x} \in \mathscr{A}^{*}(N)$ such that $\ddot{y}_{s} \in C\left(\mathscr{E}_{s}(\bar{x}, N)\right)$ for all $s$,

2. there do not exist $M \subseteq N$ and $x \in \mathscr{M}^{*}(M)$ such that $v^{i}\left(x^{*}\right)>v^{i}\left(\bar{y}^{i}\right)$ for all $i \in M$.

Unlike Definitions 5.1 and 5.2, Definition 5.3 assumes that a coalition can only use constrained feasible allocations to improve at period zero: no exchange can take place within the deviating coalition after the uncertainty has been resolved. The coalition that deviates at period zero is this confined in its choice of the consumption bundles to those that can be allocated by means of exchange of assets alone. One possible interpretation of the two-stage core is that the deviating players, being unable to foresee the consequences of their deviation, incorporate the most pessimistic scenario in their expectations: namely, that there will be no trade in period $t=1$.

The relationship between the three concepts of the sequential core is summarized by the following theorem.

\section{Theorem 5.1}

1. $\operatorname{SSC}^{*}(\mathscr{6}) \subseteq W S C(\mathscr{6})$

2. Suppose that $X_{s}^{i}$ is closed, convex and bounded from belou, $u_{s}^{i}$ is continuous and quasi-concave for all players $i$ and all states s. Then WSC(E) $\subseteq T S C(\mathscr{E})$. 
Proof. The first part of Theorem 5.1 is obvious. To prove the second part, consider an allocation $\bar{y}$ in the weak sequential core and suppose that it is not in the two-stage core. Then there exists a coalition $M \subseteq N$ and an allocation $x \in \mathcal{U}^{*}(M)$ such that $v^{i}\left(x^{i}\right)>v^{i}\left(\bar{y}^{i}\right)$ for all $i \in M$. The assumptions of the theorem guarantee that the cores of the economies $\mathscr{E}_{s}(x, M)$ are non-empty for all $s$. Choose allocations $y_{s} \in C\left(\mathscr{S}_{s}(x, M)\right)$, arbitrarily. Then $v^{i}\left(y^{i}\right) \geq v^{i}\left(x^{i}\right)>v^{i}\left(\bar{y}^{i}\right)$ for all $i \in M$. This implies, however, that $\bar{y}$ is not an element of the weak sequential core, a contradiction. We conclude that $\bar{y} \in T S C(\mathscr{E})$.

As the proof makes it clear, the inclusion of the weak sequential core in the two-stage core holds, whenever the classical cores of the economies $\mathscr{E}_{s}(x, M)$ are non-empty for all $s, M \subseteq N$, and $x \in \mathscr{A}^{*}(M)$. This is guaranteed by the conditions of the second part of Theorem 5.1. Otherwise there may be less possibilities for the deviating coalitions to form within the weak sequential core than within the two-stage core. In this case the weak sequential core need not be a subset of the two-stage core.

\subsection{Some special cases}

In this section we consider three special cases: the case where a complete set of statecontingent contracts is available, the case of a finance economy, and the case of an economy with two players.

We start with the case where for each commodity $l$ and each state of nature $s$ there is a contract specifying the delivery of commodity $l$ contingent on the occurrence of state of nature $s$. The total number of state-contingent contracts is therefore $S L$. When all of these state-contingent contracts are available, $A$ is the identity matrix. More generally, we can require the rank of the matrix $A$ to be equal $S L$. If this is indeed the case, then the weak sequential core, the strong* sequential core, and the two stage core coincide.

Theorem 5.2 Suppose that $X_{s}^{i}$ is closed, convex and bounded from below, $u_{s}^{i}$ is continuous and quasi-concave for all $i$ and $s$. If $(A)=\mathbb{R}^{S L}$, then $S S C^{*}\left(\mathscr{S}^{\circ}\right)=W S C(\mathscr{G})=\operatorname{TSC}(\mathscr{E})$.

Proof. By Theorem 5.1, SSC* $(\mathscr{E}) \subseteq W S C(\mathscr{E}) \subseteq T S C(\mathscr{E})$. And if $\langle A\rangle=\mathbb{R}^{S L}$, then $\operatorname{TSC}\left(\mathscr{E}^{*}\right) \subseteq \operatorname{SSC}^{*}\left(\mathscr{E}^{\circ}\right)$

Next we examine the case of a finance economy. A finance economy is a special case of a two-period economy, where a unique commodity is available in each state of nature: $L=1$. As we have seen in the proof of Corollary 4.1 , if $X_{s}^{i}=\mathbb{R}_{++}$. and the utility functions $u_{s}^{i}$ are all strongly monotone, then for any state $s$, coalition $M \subseteq N$ and a constrained feasible allocation $x \in \mathscr{A}^{*}(M)$ the core of the economy $C\left(\mathscr{E}_{s}(x, M)\right)$ is a one point set $\left\{x_{s}\right\}$. This observation leads to the following theorem.

Theorem 5.3 If $L=1, X_{s}^{i}=\mathbb{R}_{++4}$, and $u_{s}^{i}$ is strongly monotone for all $i \in N$ and all $s$, then $W S C\left(\mathscr{E}^{\circ}\right)=\operatorname{TSC}\left(\mathscr{C}^{a}\right)$. 
To conclude the present section we formulate a non-emptiness result for the weak sequential core in the case of an economy with two players.

Theorem 5.4 Suppose that the economy \& satisfies the following assumptions: $n=2$, the consumption sets $X_{*}^{i}$ are closed and bounded from below, and the elementary utility functions $u_{s}^{i}$ are continuous. Then the weak sequential core of the economy $\&$ is nonempty.

Proof. We shall refer to the two players as $a$ and $b$. The following notation will be useful for the proof:

$$
\begin{gathered}
X_{s}(N)=\left\{y_{s} \in \underset{i \in N}{\times} X_{s}^{i} \mid \sum_{i \in N} y_{s}^{i}=\sum_{i \in N} e_{s}^{i}\right\} \\
X(N)=\times_{s=1}^{s} X_{s}(N) \\
Z(N)=\left\{(x, y) \in \mathscr{A}^{*}(N) \times X(N) \mid \begin{array}{c}
v^{i}\left(e^{i}\right) \leq v^{i}\left(y^{i}\right), \\
u_{s}^{i}\left(x_{s}^{i}\right) \leq u_{s}^{i}\left(y_{s}^{i}\right) \\
\text { all } s=1, \ldots, S, i \in N
\end{array}\right\} .
\end{gathered}
$$

Observe that $(e, e) \in Z(N)$. Moreover, under the assumptions of Theorem 5.4, both $X(N)$ and $\mathscr{H}^{*}(N)$ are compact sets. It follows that $Z(N)$ is a compact set as well.

Let $\alpha^{a}$ and $\alpha^{b}$ be non-negative real numbers at least one of which is positive. Let $(\bar{x}, \bar{y})$ be an element of $Z(N)$ that maximizes the function

$$
\alpha^{a} v^{a}\left(y^{a}\right)+\alpha^{b} v^{b}\left(y^{b}\right)
$$

over the set $Z(N)$. In the remainder of the proof we show that the allocation $\bar{y}$ is an element of the weak sequential core.

First we show that the allocation $\tilde{y}_{s}$ belongs to the core of the ex post subeconomy $\mathscr{E}_{s \bar{s}}$, for all states $s$. Since $u_{s}^{i}\left(\vec{x}_{s}^{i}\right) \leq u_{s}^{i}\left(\vec{y}_{s}^{i}\right)$, neither player a nor player $b$ is able to improve upon allocation $\ddot{y}$ at period $t=1$, given the allocation $\bar{x}$. Now suppose that the grand coalition is able to improve upon $y$ at period $t=1$ by deviating in some state $\sigma$. Then there is an allocation $z_{\sigma} \in X_{\sigma}(N)$ such that $u_{\sigma}^{i}\left(\bar{y}_{\sigma}^{i}\right)<u_{\sigma}^{i}\left(z_{\sigma}^{i}\right)$ for both players $i=a, b$. Define the allocation $z \in X(N)$ as follows:

$$
\bar{z}_{s}^{i}= \begin{cases}z_{\infty}^{i}, & \text { if } s=\sigma \\ \bar{y}_{s}^{i}, & \text { otherwise }\end{cases}
$$

Then $u_{s}^{i}\left(\bar{y}_{s}^{i}\right) \leq u_{s}^{i}\left(\bar{z}_{s}^{i}\right)$ for $i=a, b$ with strict inequalities in state $\sigma$. It follows that $v^{i}\left(\bar{y}^{i}\right)<$ $v^{i}\left(\bar{z}^{i}\right)$ for both $i=a, b$, and that the pair $(\bar{x}, \bar{z})$ is an element of the set $Z(N)$. This contradicts the definition of $(\bar{x}, \bar{y})$.

Now we show that the pair of allocations $(\bar{x}, \bar{y})$ satisfies the second part of Definition 5.1. Since $v^{i}\left(e^{i}\right) \leq v^{i}\left(\vec{y}^{i}\right)$, neither of the two players can improve upon $\ddot{y}$ by deviating to their initial endowments at period $t=0$. Suppose that the grand coalition is able to improve upon $\vec{y}$ by deviating at period $t=0$. Let $(x, y)$ denote an improving deviation, i.e. 
a pair of allocations such that $x \in \mathscr{A}^{* *}(N), y_{s} \in C\left(\mathscr{E}_{s}(x, N)\right.$ ) for all $s$, and $v^{i}\left(\bar{y}^{i}\right)<v^{i}\left(y^{i}\right)$ for $i=a, b$. Then $y$ is an ex ante individually rational allocation. Since $y_{s}$ is individually rational in the ex post subeconomies, we must have $u_{s}^{i}\left(x_{s}^{i}\right) \leq u_{s}^{i}\left(y_{s}^{i}\right)$. We see that $(x, y)$ is an element of the set $Z(N)$. This contradicts the definition of $(\tilde{x}, \bar{y})$.

\subsection{An NTU-characterization of the weak sequential core}

It is well known that, given a static economy, one can define an associated NTU-game in such a way that the core of the underlying economy is non-empty if and only if the core of the derived N'TU-game is non-empty. Below we provide a similar criterion for non-emptiness of the weak sequential core based on an NTU-game associated with the two-period economy. This criterion for non-emptiness will be helpful in the next section.

For a given coalition $M \subseteq N$, a constrained $M$-feasible allocation $x \in \mathscr{O}^{*}(M)$ and state $s$ let $\left(M, U_{s x}\right)$ denote the NTU-game associated with the econorny $\mathscr{E}_{s}(x, M)$. That is, for a subcoalition $Q$ of $M$, we define

$$
U_{s x}(Q)=\left\{\begin{array}{l|l}
u_{s} \in \mathbb{R}^{Q} & \begin{array}{c}
\text { There exists } y_{s} \in x_{i \in Q} X_{s}^{i} \\
\sum_{i \in Q} y_{s}^{i}=\sum_{a \in Q} x_{s}^{i}, \\
u_{s}^{i} \leq u_{s}^{i}\left(y_{s}^{i}\right), i \in Q
\end{array}
\end{array}\right\} .
$$

Let $C\left(M, U_{s x}\right)$ be the core of the game $\left(M, U_{s x}\right)$. We write $C\left(M, U_{s x}\right)^{-}$to denote the set: $C\left(M, U_{s: x}\right)-\mathbb{R}_{+}^{M}$.

\section{Proposition 5.1}

1. Let $\bar{y}_{s} \in C\left(\mathscr{E}_{s}(x, M)\right)$. Then $\bar{u}_{s} \in C\left(M, U_{s:}\right)$, where $\bar{u}_{s}^{i}=u_{s}^{i}\left(\bar{y}_{s}^{i}\right)$ for all $i \in M$.

2. Let $\bar{u}_{s} \in C\left(M, U_{s x}\right)$. Then there exists $\bar{y}_{s} \in C\left(\mathscr{E}_{s}(x, M)\right)$ such that $\bar{u}_{s}^{i} \leq u u_{s}^{i}\left(\bar{u}_{s}^{i}\right)$ for all $i \in M$.

Observe that no assumptions whatsoever are made with respect to the utility functions in Proposition 5.1, and so the inequality sign in the second part of the lemra cannot in general be replaced with the equality sign. Assuming continuity and strong monotonicity of the functions $u_{s}^{i}$ and setting $X_{s}^{i}=\mathbb{R}_{+}^{L}$, one can argue indeed that for every $\tilde{u}_{s}$ in $C\left(M, U_{s x}\right)$ there exists $\vec{y}_{s}$ in $C\left(\mathscr{E}_{s}(x, M)\right)$ such that $u_{s}^{i}\left(\bar{y}_{s}^{i}\right)$ is exactly equal to $\bar{u}_{s}^{i}$ for all $i \in M$.

Define the NTU-game $(N, V)$ as follows:

$$
\begin{gathered}
V(M)=\bigcup_{x \in d^{*}(M)} V_{x}(M), \text { where } \\
V_{x}(M)=\sum_{s=1}^{S} \rho_{s} C\left(M, U_{s x}\right)^{-}
\end{gathered}
$$

for all $M \subseteq N$. Let $C(N, V)$ be the core of $(N, V)$. 


\section{Theorem 5.5}

1. Let $\tilde{y} \in W S C(\mathscr{E})$. Then $\ddot{v} \in C(N, V)$, where $\bar{y}^{i}=w^{i}\left(\bar{y}^{i}\right)$ for all $i \in N$.

2. Let $\tilde{v} \in C(N, V)$. Then there exists $\bar{y} \in W S C(\mathscr{G})$ such that $\bar{v}^{i} \leq v^{i}\left(\bar{y}^{i}\right)$ for all $i \in N$.

Proof. To prove the first part of the theorem, Let $\vec{y} \in W S C(E)$. Then there exists an $\vec{x} \in \mathscr{F}^{*}(N)$ such that $\vec{y}_{s} \in C\left(\mathscr{E}_{g}(\bar{x}, N)\right)$ for all 5 . By the first part of Proposition 5.1 , $\bar{u}_{s} \in C\left(N, U_{s, z}\right)$, where $\bar{u}_{s}^{i}=u_{s}^{i}\left(\bar{y}_{s}^{i}\right)$ for all $i \in N$ and all s. Therefore, $\tilde{v} \in V_{x}(N) \subseteq V(N)$, where $\bar{v}=\sum_{s=1}^{S} \rho_{s} \vec{u}_{s}$, and so $\bar{v}^{i}=v^{i}\left(\bar{y}^{i}\right)$ for all $i \in N$.

Suppose that there exist $M \subseteq N$ and $v \in V(M)$ with $\vec{v}^{i}<v^{i}$ for all $i \in M$. Then there exist $x \in \mathscr{R}^{*}(M)$ and $u_{s} \in C\left(M, U_{s x}\right)$ for all $s$ such that $u^{2} \leq \sum_{s=1}^{S} \rho_{s} u_{s}^{i}$ for all $i \in M$. By the second part of Proposition 5.1, there exist $y_{s} \in C\left(\mathscr{E}_{s}(x, M)\right)$ such that $u_{s}^{i} \leq u_{s}^{i}\left(y_{s}^{i}\right)$ for all sand $i \in M$. Hence, $v^{i}\left(y^{i}\right)<v^{i}\left(y^{i}\right)$ for all $i \in M$. This implies, however, that $\bar{y}$ is not in the weak sequential core of the economy $s$ a contradiction. We conclude therefore that $\tilde{v} \in \mathrm{C}(\mathrm{N}, \mathrm{V})$.

To demonstrate the second part of the theorem, let $\bar{v} \in C(N, V)$. Then there exist

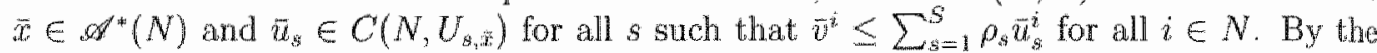
second part of Proposition 5.1, there exist $\bar{y}_{s} \in C\left(\mathscr{E}_{s}\left(\bar{x}_{3}, N\right)\right)$ such that $\bar{u}_{s}^{i} \leq u_{s}^{i}\left(\bar{y}_{s}^{i}\right)$ for all $s$ and $i \in N$. So, $\bar{w}^{i} \leq v^{i}\left(y^{i}\right)$ for all $i \in N$.

Suppose that thene exist $M \subseteq N, x \in \mathscr{A}^{*}(M)$, and $y_{s} \in C\left(\mathscr{E}_{s}(x, M)\right)$ for all such that $v^{i}\left(y^{i}\right)<v^{i}\left(y^{i}\right)$ for all $i \in M$. Then, by the first part of Proposition 5.1, $u_{s} \in C\left(M, U_{s x}\right)$ for all $s$, where $u_{s}^{i}=u_{s}^{i}\left(y_{s}^{i}\right)$ for all $i \in M$. Therefore, $v \in V_{x}(M) \subseteq V(M)$, where $v=\sum_{s=1}^{s} \rho_{s} u_{s}$, and so $v^{i}=v^{i}\left(y^{i}\right)$ for all $i \in M$. It follows that $\bar{v}^{i}<v^{i}$ for all $i \in M$. So, $\bar{v} \notin C(N, V)$, a contradiction. We conclude therefore that $\bar{y} \in W S C(\mathscr{E})$.

Example 5.1 Consider an economy $\mathscr{E}$ where all players have identical consumption sets and identical state-independent elementary utility functions

$$
\begin{array}{r}
X_{s}^{i}=\mathbb{R}_{s+1}^{L} \\
u_{s}^{i}\left(y_{s}^{i}\right)=\frac{1}{L} \sum_{l=1}^{L} \ln \left(y_{s t}^{i}\right), \quad y_{s}^{i} \in X_{s}^{i}
\end{array}
$$

Wo derwe the core of the game $\left(M, U_{s a n}\right)$ for this special case.

Observe that an allocation $y_{s} \in X_{s}$ is Pareto efficient in the ex post subeconomy $\mathscr{G}_{s}(x, Q)$ if and only if there exist real numbers $t_{s}>0$ for all players $\dot{x} \in Q$ such that $\sum_{i \in Q} t_{s}^{i}=1$ and $y_{s}^{i}=t_{s}^{q} x_{s}^{Q}$, where $x_{s}^{Q}=\sum_{j e q} x_{s}^{j}$ denotes the aggregate endowment of coalition $Q$. It follows that the set $U_{a x}(Q)$ can be witten as

$$
\begin{aligned}
& U_{s, x}(Q)=\left\{\begin{array}{l|l}
\exists t_{s}^{i}>0, i \in Q: \sum_{i \in Q} t_{s}^{i}=1 \\
\exp \left(u_{s}^{i}\right) \leq t_{s}^{i} \alpha_{s x}(Q), i \in Q
\end{array}\right\} \\
& =\left\{u_{s} \in \mathbb{R}^{Q} \mid \sum_{i \in Q} \exp \left(u_{s}^{i}\right) \leq \alpha_{s x}(Q)\right\}
\end{aligned}
$$


where the numbers $\alpha_{5 x}(Q)$ are defined by the equation

$$
\alpha_{s, x}(Q)=\left(\prod_{i=1}^{L} x_{s i}^{Q}\right)^{\frac{1}{d}}
$$

Now the core of the game $\left(M, U_{s x}\right)$ is given by the set

$$
C\left(M, U_{s i}\right)=\left\{\begin{array}{l|l}
u_{s} \in \mathbb{R}^{M} & \begin{array}{c}
\sum_{i \in M} \exp \left(u_{s}^{i}\right)=\alpha_{s i x}(M) \\
\sum_{i \in Q} \exp \left(u_{s}^{i}\right) \geq \alpha_{s x}(Q), Q \subset M
\end{array}
\end{array}\right\} .
$$

If we set $\alpha_{s x}(\emptyset)$ equal to 0 , then the core of $\left(M, U_{s x}\right)$ can be rewritten as

$$
C\left(M, U_{s x}\right)=\left\{\begin{array}{l|c}
u_{s} \in \mathbb{R}^{M} & \begin{array}{c}
\sum_{i \in M} \exp \left(u_{s}^{i}\right)=\alpha_{s x}(M) \\
\sum_{i \in Q} \exp \left(u_{s}^{i}\right) \leq \alpha_{s x}(M)-\alpha_{s x}(M \backslash Q), Q \subset M
\end{array}
\end{array}\right\} .
$$

Therefore,

$$
\begin{gathered}
C\left(M, U_{s x}\right)^{-}= \\
=\left\{u_{s} \in \mathbb{R}^{M} \mid \sum_{i \in Q} \exp \left(u_{s}^{i}\right) \leq \alpha_{\alpha \Delta x}(M)-\alpha_{s, x}(M \backslash Q), Q \subseteq M\right\} .
\end{gathered}
$$

\subsection{Examples}

The purpose of the two examples reported below is to demonstrate some limitations of the concept of the weak sequential core. In Example 5.2 an economy with three agents and no dssets is shown to have an empty weak sequential core. In contrast, the conony in Example 5.3, also with three agents and no assets, has a non-emply weak sequential core. However, the weak sequential core becomes empty, when an appropriately specified inset is introduced into the economy.

Example 5.2 Consider an economy of with three players referred to as $a, b$, and $c$ having identical consumption sets and identical state independent elementary utility functions defined by Equations (5.1)-(5.2). Suppose that, there are two states, three commodities in each state and no assets: $S=2, L=3$ and $J=0$. The initial endowments in the two states of nature are given by

$$
\begin{aligned}
& e_{1}=\left(e_{1}^{a}, e_{1}^{b}, e_{1}^{c}\right)=\left(\begin{array}{lll}
1 & \epsilon & 1 \\
\epsilon & 1 & 1 \\
\epsilon & 1 & 1
\end{array}\right) \\
& e_{2}=\left(e_{2}^{a}, e_{2}^{b}, e_{2}^{c}\right)=\left(\begin{array}{lll}
\epsilon & 1 & 1 \\
1 & \epsilon & 1 \\
1 & \epsilon & 1
\end{array}\right) .
\end{aligned}
$$


Claim 5.1 The weal sequential core of the economy $\mathscr{E}$ is empty whenever $0<\epsilon \leq 0.021$.

Proof. Before we come to a formal proof let us give some intuition for the emptiness result.

In Figure 5.1 the set $C\left(\{a, b\}, U_{s, e}\right)$ and the projection of the set $C\left(N, U_{s, e}\right)$ onto the space of utilities for agents $a$ and $b$ in the state $s=1$ are depicted. Figure 5.2 depicts the same objects for the state $s=2$. Figure 5.3 depicts the set $V(\{a, b\})$ and the projection of the set $V(\{a, b, c\})$ onto $\mathbb{R}^{\{a, b\}}$.

Observe that the vectors of the aggregate endowments $e_{s}^{a}+e_{s}^{b}$ owned by agents $a$ and $b$ are equal across the states. Since the elementary utility functions of both agents $a$ and $b$ are state-independent, the feasibility sets $U_{1, e}(\{a, b\})$ and $U_{2, e}(\{a, b\})$ in the two states of the world coincide. As $\epsilon$ gets smaller, the conditions of individual rationality for agents $a$ and $b$ become negligible. Therefore, the cores $C\left(\{a, b\}, U_{s, e}\right)$ are "almost" (up to the vanishing conditions of individual rationality) identical across the states of nature. This indicates the possibility of credible agreements within a coalition $\{a, b\}$ upon the state-independent (certain) allocation of utilities.

Due to the acute choice of values for the initial endowments, the core of the game $\left(\{a, b, c\}, U_{s, e}\right)$, when projected into the space $\mathbb{R}^{\{a, b\}}$, is a subset of $C\left(\{a, b\}, U_{s, e}\right)$. Moreover, the sets $C\left(\{a, b, c\}, U_{1, e}\right)$ and $C\left(\{a, b, c\}, U_{2, e}\right)$ are disjoint: the grand coalition is not able to agree upon any allocation of commodities under which agents $a$ and $b$ would enjoy state-indepencient utilities.

The risk aversion of the agents $a$ and $b$ reflected here by the strict convexity of the sets $U_{1, e}(\{a, b\})$ and $U_{2, e}(\{a, b\})$ implies that the coalition $\{a, b\}$ can improve upon any expected utility profile in the set $V(N)$. That is, $\left(v^{a}, v^{b}\right) \in \operatorname{int} V(\{a, b\})$ for all $v \in V(\{a, b, c\})$. Below we give a formal proof of this result.

Let $(N, V)$ be the NTU-game as defined in Section 5.5. We show that the core of $(N, V)$ is empty whenever the following inequalities hold:

$$
\begin{aligned}
& 0<\epsilon<1, \\
& 2+\epsilon-(4(1+\epsilon))^{\frac{1}{3}}<(4(1+\epsilon))^{\frac{1}{3}}-1, \\
& 2+\epsilon-\left(2(1+\epsilon)^{2}\right)^{\frac{1}{3}}<1+\epsilon-\epsilon^{\frac{1}{3}} .
\end{aligned}
$$

Notice that each $e$ in the interval $(0 ; 0.021]$ satisfies the inequality constraints.

Table 5.1 reports the values for $\alpha_{s e}(Q)$ as defined by Equation (5.3). Using these values and the formula (5.4) in Section 5.5 , it is easy to see that for coalition $\{a, b\}$

$$
\begin{gathered}
C\left(\{a, b\}, U_{1, e}\right)^{-}= \\
\left\{u_{1} \in \mathbb{R}^{\{a, b)} \mid \begin{array}{ll}
\exp \left(u_{1}^{a}\right)+\exp \left(u_{1}^{b}\right) & \leq 1+\epsilon \\
\exp \left(u_{1}^{a}\right) & \leq 1+\epsilon-\epsilon^{\frac{1}{3}} \\
\exp \left(u_{1}^{b}\right) & \leq 1+\epsilon-\epsilon^{\frac{2}{3}}
\end{array}\right\}
\end{gathered}
$$




\begin{tabular}{lll}
\multicolumn{3}{c}{ Table 5.1: The values for $\alpha_{\text {se }}(Q)$} \\
\hline & $s=1$ & $s=2$ \\
$Q=\{a\}$ & $\epsilon^{\frac{2}{3}}$ & $\epsilon^{\frac{1}{3}}$ \\
$Q=\{b\}$ & $\epsilon^{\frac{1}{3}}$ & $\epsilon^{\frac{2}{3}}$ \\
$Q=\{c\}$ & $\mathbb{1}$ & 1 \\
$Q=\{a, b\}$ & $\mathbb{1}+\epsilon$ & $1+\epsilon$ \\
$Q=\{a, c\}$ & $\left(2(1+\epsilon)^{2}\right)^{\frac{1}{3}}$ & $\left(4(1+\epsilon)^{\frac{1}{3}}\right.$ \\
$Q=\{b, c\}$ & $(4(1+\epsilon))^{\frac{1}{3}}$ & $\left(2(1+\epsilon)^{2}\right)^{\frac{1}{3}}$ \\
$Q=\{a, b, c\}$ & $2+\epsilon$ & $2+\epsilon$ \\
\hline
\end{tabular}

and

$$
\begin{gathered}
C\left(\{a, b\}, U_{2, e}\right)^{-}= \\
\left\{u_{2} \in \mathbb{R}^{\{a, b\}} \mid \begin{array}{ll}
\exp \left(u_{2}^{a}\right)+\exp \left(u_{2}^{b}\right) & \leq 1+\epsilon \\
\exp \left(u_{2}^{a}\right) & \leq 1+\epsilon-\epsilon^{\frac{2}{3}} \\
\exp \left(u_{2}^{b}\right) & \leq 1+\epsilon-\epsilon^{\frac{1}{3}}
\end{array}\right\}
\end{gathered}
$$

For the grand coalition

$$
\begin{aligned}
& C\left(N, U_{1, e}\right)=
\end{aligned}
$$

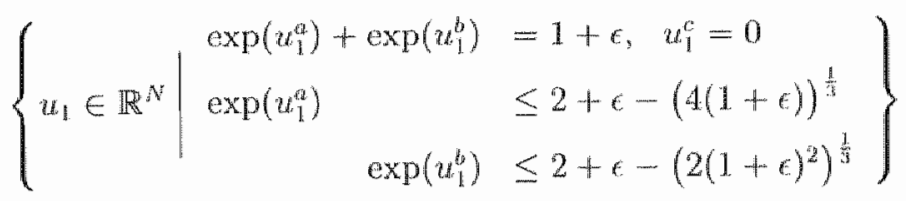

and

$$
\begin{gathered}
C\left(N, U_{2, e}\right)= \\
\left\{u_{2} \in \mathbb{R}^{N} \mid \begin{array}{rl}
\exp \left(u_{2}^{a}\right)+\exp \left(u_{2}^{b}\right) & =1+\epsilon, u_{2}^{c}=0 \\
\exp \left(u_{2}^{a}\right) & \leq 2+\epsilon-\left(2(1+\epsilon)^{2}\right)^{\frac{1}{3}} \\
\exp \left(u_{2}^{b}\right) & \leq 2+\epsilon-(4(1+\epsilon))^{\frac{1}{3}}
\end{array}\right\}
\end{gathered}
$$

We proceed by demonstrating that the projection of $V(N)$ onto the space of atilitics for agents $a$ and $b$ lies entirely in the interior of the set $V(\{a, b\})$.

First we show that for each point $u_{s}$ in $C\left(N, U_{s, e}\right)$ the inequalities $u_{1}^{a}<u_{2}^{a}$ and $u_{1}^{b}>u_{2}^{b}$ hold. Take any $u_{s} \in C\left(N, U_{s, e}\right), s=1,2$. One can see from Equations (5.5) and (5.6) that 
the following inequalities hold:

$$
\begin{aligned}
& \exp \left(u_{1}^{a}\right) \leq 2+\epsilon-(4(1+\epsilon))^{\frac{1}{3}} \\
& \exp \left(u_{2}^{a}\right) \geq(4(1+\epsilon))^{\frac{1}{3}}-1 .
\end{aligned}
$$

The inequality constraints imposed upon $\epsilon$ therefore guarantee that $\exp \left(u_{1}^{a}\right)<\exp \left(u_{2}^{a}\right)$. The equations $\exp \left(u_{s}^{a}\right)+\exp \left(u_{s}^{b}\right)=1+\varepsilon, s=1,2$ imply that $\exp \left(u_{1}^{b}\right)>\exp \left(u_{2}^{b}\right)$.

Now we demonstrate that for each element $v$ of the set $V(N)$ the pair $\left(v^{a}, v^{b}\right)$ belongs to the interior of the sets $C\left(\{a, b\}, U_{s, e}\right)-$ for $s=1$ and $s=2$. It suffices to show that for any $v \in V(N)$ the following inequalities hold true:

$$
\begin{aligned}
\exp \left(v^{a}\right)+\exp \left(v^{b}\right) & <1+\epsilon \\
\exp \left(v^{i}\right) & <1+\epsilon-\epsilon^{\frac{1}{3}}, \quad i=a, b .
\end{aligned}
$$

Choose $u_{s} \in C\left(N, U_{s, e}\right)$ so that $v \leq \rho_{1} u_{1}+\rho_{2} u_{2}$. The fact that $u_{1}^{a} \neq u_{2}^{a}$, the strict convexity of the exponential function, and the equations $\exp \left(u_{s}^{a}\right)+\exp \left(u_{s}^{b}\right)=1+\epsilon$ yield the inequalities

$$
\begin{array}{r}
\exp \left(v^{a}\right)+\exp \left(v^{b}\right) \leq \\
\leq \exp \left(\rho_{1} u_{1}^{a}+\rho_{2} u_{2}^{a}\right)+\exp \left(\rho_{1} u_{1}^{b}+\rho_{2} u_{2}^{b}\right)< \\
<\rho_{1} \exp \left(u_{1}^{a}\right)+\rho_{2} \exp \left(u_{2}^{a}\right)+\rho_{1} \exp \left(u_{1}^{b}\right)+\rho_{2} \exp \left(u_{2}^{b}\right)= \\
=\rho_{1}\left(\exp \left(u_{1}^{a}\right)+\exp \left(u_{1}^{b}\right)\right)+\rho_{2}\left(\exp \left(u_{2}^{a}\right)+\exp \left(u_{2}^{b}\right)\right)= \\
=1+\varepsilon
\end{array}
$$

Since $u_{1}^{a}<u_{2}^{a}$, we have the inequality $\exp \left(v^{a x}\right)<\exp \left(u_{2}^{a}\right)$. Similarly, from and $u_{1}^{b}>u_{2}^{b}$ we obtain the inequality $\exp \left(v^{b}\right)<\exp \left(u_{1}^{b}\right)$. As is evident from (5.5) and (5.6), both $\exp \left(u_{2}^{a}\right)$ and $\exp \left(u_{1}^{b}\right)$ are less than or equal to

$$
2+\epsilon-\left(2(1+\epsilon)^{2}\right)^{\frac{1}{3}}
$$

which, under the inequality constraints imposed upon $\epsilon$, is less than $1+\epsilon-\epsilon^{1 / 3}$.

We conclude that for each element $v$ of $V(N)$, the pair $\left(v^{(a}, v^{b}\right)$ belongs to the interior of the set $V(\{a, b\})$.

Example 5.3 Consider an economy $\mathscr{f}$ with three players $a, b$, and $c$ having identical consumption sets and identical state-independent elementary utility functions given by Equations (5.1) -(5.2). Suppose that there are two equally probable states, and two commodities in each state: $S=2$, and $L=2$. The initial endowments are given by the equations

$$
\begin{aligned}
& e_{1}=\left(e_{1}^{a}, e_{1}^{b}, e_{1}^{c}\right)=\left(\begin{array}{lll}
1 & \epsilon & 1 \\
1 & \epsilon & 1
\end{array}\right) \\
& e_{2}=\left(e_{2}^{a}, e_{2}^{b}, e_{2}^{c}\right)=\left(\begin{array}{lll}
1 & 1 & \varepsilon \\
1 & \epsilon & 1
\end{array}\right)
\end{aligned}
$$


Suppose that there are no assets in the economy of.

Clam 5.2 The weat sequential core of the economy is given by the set

$$
W S C(\mathscr{E})=\left\{e_{1}\right\} \times C\left(\mathscr{E}_{2}(e, N)\right)
$$

Proof. Note that for any coalition $M \subseteq N$ the core of the economy $\mathscr{E}_{1}(e, M)$ contains a unique allocation: the initial endowments. That is,

$$
C\left(\mathscr{E}_{1}(e, M)\right)=\left\{\left(e_{1}^{\mathrm{i}}\right)_{i \in M}\right\}
$$

Therefore, an allocation $\bar{y}$ is an element of the weak sequential core if and only if $y_{1}=e_{1}$. $y_{2} \in C\left(\mathscr{C}_{2}(e, N)\right)$, and there exist no coalition $M \subseteq N$ and no allocation $y \in x_{\text {ieM }} X^{*}$ such that $y_{1}^{i}=e_{1}^{i}$ for all $i \in M, y_{2} \in C\left(\mathscr{O}_{2}(e, M)\right)$, and $v^{i}\left(y^{i}\right)>v^{i}\left(\bar{y}^{i}\right)$ for $i \in M$. The latker inequality may be replaced by $u_{2}^{i}\left(y_{2}^{i}\right)>u_{2}^{i}\left(\bar{y}_{2}^{i}\right)$. Therefore, the set of weak sequential core allocations $\left(\bar{y}_{1}, \bar{y}_{2}\right)$ is exactly $\left\{e_{1}\right\} \times C\left(\mathscr{b}_{2}(e, N)\right)$.

Suppose we introduce an a single asset to the economy with the payoffs given by the vector $A^{1}=(1,0,0,0)$. This asset is a contract for the delivery of commodity $l=1$ in stite $s=1$.

Clam 5.3 The weak sequential core of the economy of with an asset is empty whenever $0<\epsilon \leq 0.038$.

Proof. Let $(N, V)$ be the NTU-game defined as in the Section 5.5 . We show that the core of the game $(N, V)$ is empty, whenever the following inequalities hold:

$$
\begin{aligned}
& 0<\epsilon<1 \\
& \frac{1}{1-\epsilon^{\frac{1}{2}}}+\epsilon^{\frac{1}{2}}<(2+2 \epsilon)^{\frac{1}{2}} \\
& 1+\left(2+\epsilon-(2+2 \epsilon)^{\frac{1}{2}}\right)^{2}<(2+2 \epsilon)^{\frac{1}{2}} .
\end{aligned}
$$

Observe that each $\epsilon$ in the interval $(0,0.038]$ satisfies the inequality constraints.

The proof is split in two parts. First we construct sone of the feasible expected utility tuples - subsets of or isolated points in $V(Q)$ - for varions sub-coalitions $Q$ of $N$. Then we that the core of $(N, V)$ is enapty.

\section{Part 1}

Notice first that for any coalition $Q \subseteq N$ and any constrained $Q$ foasible allocation $x \in$ $\mathscr{A}^{*}(Q)$ it holds that $x_{2}^{i}=e_{2}^{i}$ for all $i \in Q$. In what follows $x$ is defined by its component $x_{1}$, corresponding to the first state of the world.

For the singletons $\{a\}$ and $\{c\}$ we have

$$
V(\{a\})=(-\infty ; 0), \text { and }
$$




$$
V(\{c\})=\left(-\infty ; \ln \epsilon^{\frac{1}{4}}\right] .
$$

For the coalition $\{b, c\}$ it holds that

$$
\begin{aligned}
& C\left(\{b, c\}, U_{2, e}\right)^{-}= \\
& \left\{u_{2} \in \mathbb{R}^{\{b, c]} \mid \begin{array}{ll}
\exp \left(u_{2}^{b}\right)+\exp \left(u_{2}^{c}\right) & \leq 1+\epsilon \\
\exp \left(u_{2}^{b}\right) & \leq 1+\epsilon-\epsilon^{\frac{1}{2}} \\
\exp \left(u_{2}^{c}\right) & \leq 1+\epsilon-\epsilon^{\frac{1}{2}}
\end{array}\right\}
\end{aligned}
$$

Let the allocation $x \in \mathscr{A}^{*}(\{b, c\})$ be defined by

$$
x_{1}=\left(x_{1}^{b}, x_{1}^{c}\right)=\left(\begin{array}{ll}
1 & \epsilon \\
\epsilon & 1
\end{array}\right) .
$$

Since $x_{1}=x_{2}=\left(e_{2}^{b}, e_{2}^{c}\right)$, we know that the three sets $C\left(\{b, c\}, U_{1, x}\right)^{-}, C\left(\{b, c\}, U_{2, x}\right)^{--}$, and $C\left(\{b, c\}, U_{2, e}\right)^{-}$coincide. It follows that $V_{x}(\{b, c\})$ coincides with each of these three sets. Thus,

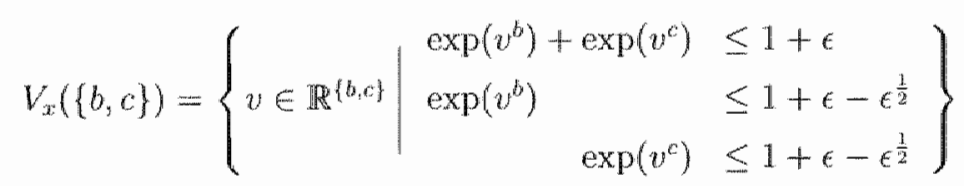

Consider next coalition $\{a, b\}$. Let the allocation $x \in \mathscr{A}^{*}(\{a, b\})$ be defined by

$$
x_{1}=\left(x_{1}^{a}, x_{1}^{b}\right)=\left(\begin{array}{ll}
\epsilon & 1 \\
1 & \epsilon
\end{array}\right) .
$$

Then

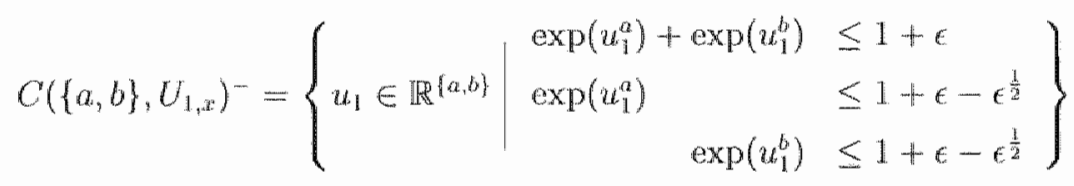

and

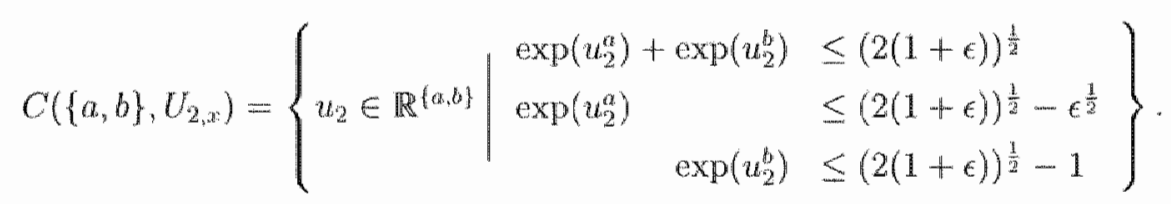

Define $u_{s} \in \mathbb{R}^{\{a, b\}}$ as follows:

$$
\begin{gathered}
\left(u_{1}^{a}, u_{1}^{b}\right)=\left(\ln \left(1-\epsilon^{\frac{1}{2}}\right), \ln \left(\epsilon^{\frac{1}{2}}\right)\right) \\
\left(u_{2}^{a}, u_{2}^{b}\right)=\left(-\ln \left(1-\epsilon^{\frac{1}{2}}\right), \ln \left(\epsilon^{\frac{1}{2}}\right)\right)
\end{gathered}
$$


Under the inequality constraints imposed upon $\epsilon, u_{s}$ lies in the interior of the set $C\left(\{a, b\}, v_{s}\right)^{-}$ for $s=1,2$. Therefore,

$$
0.5 u_{1}+0.5 u_{2}=\left(0, \ln \epsilon^{\frac{1}{2}}\right) \in \operatorname{in} V_{x}(\{a, b\}) .
$$

For the coalition $\{a, c\}$ it holds that

$$
\begin{aligned}
& C\left(\{a, c\}, U_{1, e}\right)=\{(0,0)\}
\end{aligned}
$$

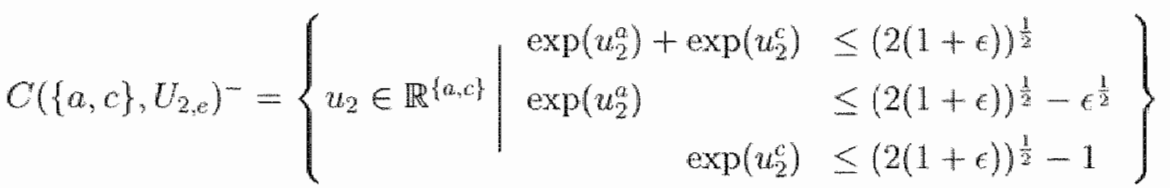

Define $u_{s} \in \mathbb{R}^{\{a, c\}}$ as follows:

$$
\begin{aligned}
& \left(u_{1}^{a}, u_{1}^{c}\right)=(0,0) \\
& \left(u_{2}^{a}, u_{2}^{c}\right)=\left(0,2 \ln \left(2+\epsilon-(2+2 \epsilon)^{\frac{1}{2}}\right)\right) .
\end{aligned}
$$

Under the inequality constraints imposed upon $\epsilon$ it holds that $w_{2}$ lies in the interior of the set $C\left(\{a, c\}, U_{2, e}\right)$. Therefore,

$$
0.5 u_{1}+0.5 u_{2}=\left(0, \ln \left(2+\epsilon-(2+2 \epsilon)^{\frac{1}{2}}\right)\right) \in \operatorname{in} t V(\{a, c\}) .
$$

Finally, consider the grand coalition. It holds that

$$
\begin{aligned}
& C\left(N, U_{2, e}\right)^{-}= \\
&\left\{u_{2} \in \mathbb{R}^{N} \mid \begin{array}{rl}
\exp \left(u_{2}^{b}\right)+\exp \left(u_{2}^{c}\right) & \leq 1+\epsilon, u_{2}^{u} \leq 0 \\
\exp \left(u_{2}^{b}\right) & \leq 2+\epsilon-(2+2 \epsilon)^{\frac{1}{2}} \\
\exp \left(u_{2}^{c}\right) & \leq 2+\epsilon-(2+2 \epsilon)^{\frac{1}{2}}
\end{array}\right\}
\end{aligned}
$$

For any $\bar{x} \in \mathscr{A}^{*}(N)$ and for each state $s$,

$$
U_{s, x}(N)=\left\{u_{s} \in \mathbb{R}^{N} \mid \exp \left(u_{s}^{a}\right)+\exp \left(u_{s}^{(s)}\right)+\exp \left(u_{s}^{c}\right) \leq 2+\epsilon\right\} .
$$

It follows from (5.14) that

$$
V(N) \subseteq\left\{v \in \mathbb{R}^{N} \mid \exp \left(v^{a}\right)+\exp \left(v^{b}\right)+\exp \left(v^{c}\right) \leq 2+c\right\} .
$$




\section{Part 2}

Let $\bar{v}$ be an element of $C(N, V)$. Let $\ddot{x} \in \mathscr{A}^{*}(N)$ and let $\bar{u}_{s} \in C\left(N, U_{s, \dot{x}}\right)^{-\cdots}$ be such that $\bar{v}=0.5 \bar{u}_{1}+0.5 \bar{u}_{2}$.

Step 1 We show that

$$
\exp \left(\bar{u}_{1}^{b}\right)+\exp \left(\bar{u}_{1}^{c}\right) \geq 1+\epsilon
$$

It suffices to demonstrate that if $\exp \left(\bar{u}_{1}^{b}\right)+\exp \left(\bar{u}_{1}^{c}\right)<1+\epsilon$, then coalition $\{b, c\}$ has a credible deviation from $v$.

Choose a utility profile $\hat{u}_{1} \in \mathbb{R}^{\{b, c\}}$ such that

$$
\begin{array}{r}
\exp \left(\hat{u}_{1}^{b}\right)+\exp \left(\hat{u}_{1}^{c}\right)=1+\epsilon \\
\left(\hat{u}_{1}^{b}, \hat{u}_{1}^{c}\right)>>\left(\bar{u}_{1}^{b}, \bar{u}_{1}^{c}\right) .
\end{array}
$$

Let the allocation $x \in \mathscr{A}^{*}(\{b, c\})$ be defined by

$$
\begin{aligned}
x_{1} & =\left(x^{b}, x^{c}\right)=\left(\begin{array}{cc}
\epsilon+\theta & 1-\theta \\
\epsilon & 1
\end{array}\right), \text { where } \\
\theta & = \begin{cases}\frac{\exp \left(2 \hat{u}_{1}^{b}\right)}{\epsilon}-\epsilon & \text { if } \hat{u}_{1}^{c} \geq 0 \\
1-\exp \left(2 \hat{u}_{1}^{c}\right), & \text { otherwise. }\end{cases}
\end{aligned}
$$

We leave it to the reader to show that $x_{11}^{i}>0, i=b, c$, and that

$$
u_{1}^{i} \geq u_{1}^{i}\left(x_{1}^{i}\right), \quad i \in\{b, c\} .
$$

It follows from Equations (5.17)-(5.18) that $\hat{u}_{1} \in C\left(\{b, c\}, U_{1 x}\right)$, and therefore

$$
\left(\bar{u}_{1}^{b}, \bar{u}_{1}^{c}\right) \in \operatorname{int} C\left(\{b, c\}, U_{1 x}\right)^{-} \text {. }
$$

Comparing Equations (5.9) and (5.13) we notice that the inclusion $\bar{u} \in C\left(N, U_{2 w}\right)$ implies the inclusion

$$
\left(\bar{u}_{2}^{b}, \bar{u}_{2}^{c}\right) \in C\left(\{b, c\}, U_{2 e}\right)^{-} \text {. }
$$

Finally, formulas (5.19) and (5.20) lead to the conclusion that $\left(\bar{v}^{b}, \bar{v}^{c}\right) \in \operatorname{int} V_{x}(\{b, c\})$.

Step 2 Wo show that

$$
\begin{array}{r}
\bar{u}_{s}^{a}=\bar{v}^{a}=0, s=1,2, \\
\exp \left(\bar{u}_{s}^{b}\right)+\exp \left(\bar{u}_{s}^{c}\right) \leq 1+\epsilon, s=1,2 .
\end{array}
$$

It follows from (5.13) that $\bar{u}_{2}^{n} \leq 0$. The fact that $\bar{u}_{1} \in U_{1 \bar{x}}(N)$ (see (5.14)) together witl Equation (5.16) imply that $\dddot{u}_{1}^{a} \leq 0$. This gives $\bar{v}^{a} \leq 0$. On the other hand, the requirement that $\bar{v}^{a}$ be outside int $V(\{a\})$ implies $\bar{v}^{a} \geq 0$ (see (5.7)). Thus we proved (5.21). Equations 
(5.22) are now implied by (5.21) and the fact that $w_{s} \in U_{s, x}(N)$ (see (5.14)).

Step 3 We show that

$$
\exp \left(\tilde{v}^{b}\right)+\exp \left(\bar{v}^{c}\right)=1+\epsilon
$$

From. (5.15) and the fact that $\bar{v}^{a}=0$ it follows that

$$
\exp \left(\bar{v}^{b}\right)+\exp \left(\bar{v}^{c}\right) \leq 1+\epsilon .
$$

The requirement that the utility profile $\left(v^{b}, \bar{v}^{c}\right)$ be outside the interior of the set defined by (5.10) implies that at least one of the following inequalities are satisfied:

$$
\begin{aligned}
\exp \left(\bar{v}^{b}\right)+\exp \left(\bar{v}^{c}\right) & \geq 1+\epsilon \\
\exp \left(\bar{v}^{b}\right) & \geq 1+\epsilon-\epsilon^{\frac{1}{2}} \\
\exp \left(\bar{v}^{c}\right) & \geq 1+\epsilon-\epsilon^{\frac{1}{2}} .
\end{aligned}
$$

If inequality (5.26) were satisfied, then, together with $(5.24)$, it would give $\exp \left(\bar{v}^{c}\right) \leq e^{\frac{3}{2}}$. Then $\bar{v}^{c}$ would lie in the interior of the set $V(\{c\})$ (see (5.8)), which cannot be the case.

If inequality (5.27) were satisfied, then, together with $(5.24)$, it would give $\exp \left(0^{\text {th }}\right) \leq \epsilon^{\frac{1}{2}}$. Hence, $\left(\bar{v}^{a z}, \vec{v}^{b}\right) \leq\left(0\right.$, In $\left.\epsilon^{\frac{1}{2}}\right)$. Inclusion (5.11) would then imply that the utility tuple $\left(\bar{v}^{a}, \bar{v}^{b}\right)$ lie in the interior of the set $V(\{a, b\})$, which cannot be the case.

Since both $(5.26)$ and $(5.27)$ are violated, (5.25) must be satisfied. Equations (5.24) and $(5.25)$ yield $(5.23)$.

Step 4 We show that

$$
\left(\bar{u}_{1}^{b}, \bar{u}_{1}^{c}\right)=\left(\bar{u}_{2}^{b}, \bar{u}_{2}^{c}\right)
$$

If this equality was violated, then, due to the strict convexity of the exponential function and inequalities $(5.22)$

$$
\begin{aligned}
\exp \left(\bar{v}^{b}\right)+\exp \left(\bar{v}^{c}\right) & <0.5 \exp \left(\bar{u}_{1}^{b}\right)+0.5 \exp \left(\bar{u}_{2}^{b}\right)+0.5 \exp \left(\bar{u}_{1}^{c}\right)+0.5 \exp \left(u_{2}^{c}\right) \\
& \leq 1+\epsilon .
\end{aligned}
$$

This last inequality, however, would contradict $(5.23)$.

Step 5 We show that $\left(\bar{v}^{2}, v^{c}\right) \in \operatorname{int} V(\{a, c\})$.

Since $\bar{u}_{2} \in C\left(N, U_{2, e}\right)$, (see (5.13)) $\bar{u}_{2}^{c}$ satisfies

$$
\exp \left(\bar{u}_{2}^{c}\right) \leq 2+\epsilon-(2+2 c)^{\frac{1}{2}} .
$$

We know from the previous step that $\bar{u}_{2}^{c}=\bar{v}^{c}$. Hence,

$$
\left(\bar{v}^{c}, \bar{v}^{c}\right) \leq\left(0 ; \ln \left(2+\epsilon-(2+2 \epsilon)^{\frac{1}{2}}\right)\right) \text {. }
$$

The inclusion (5.12) implies that the utility tuple $\left(\tilde{v}^{a}, \bar{v}^{c}\right)$ belongs to the interior of the sot $V(\{a, c\})$, leading to a contradiction. 

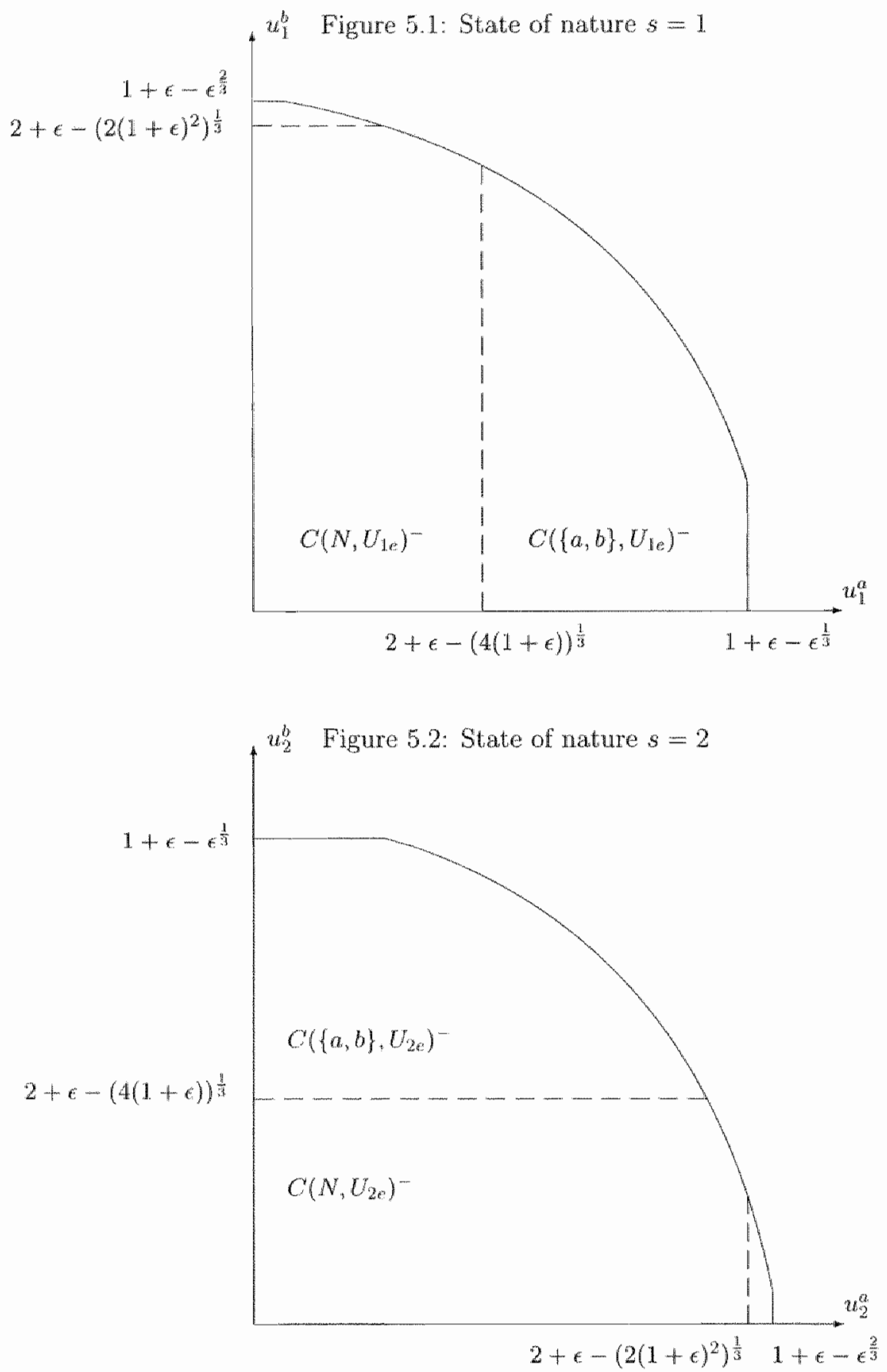


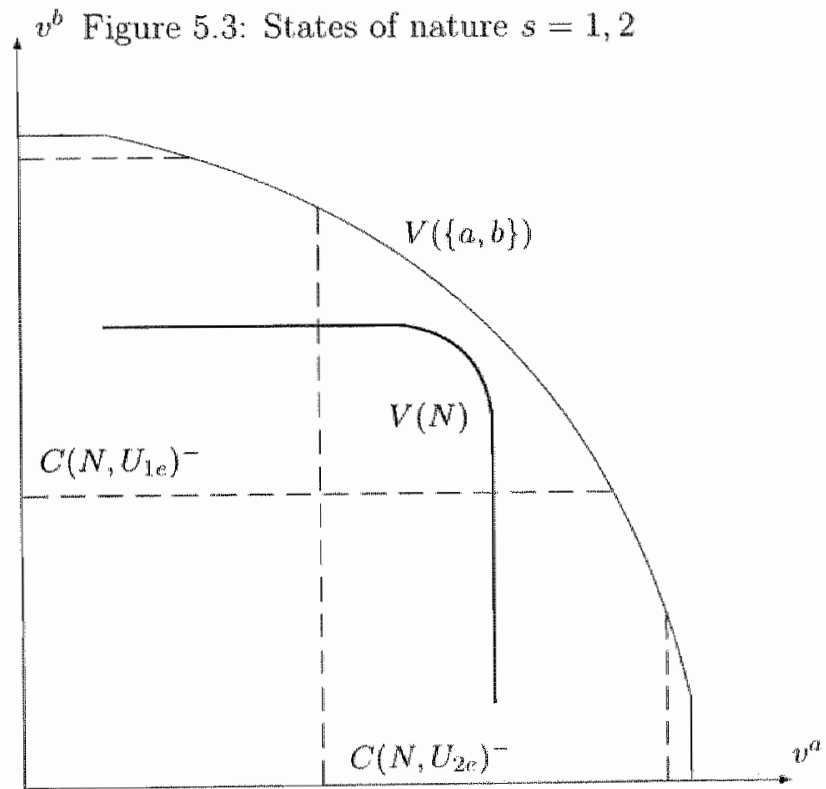





\section{Chapter 6}

\section{The Strong Sequential Core in a Stationary Exchange Economy ${ }^{1}$}

\subsection{Introduction}

This chapter continues with the subject introduced earlier in Chapter 4: namely, the strong sequential core. We have seen that, in the context of a two-period exchange economy, robust examples of an empty strong sequential core can be constructed. Moreover, in the class of two-period finance economies where the difference between the number of states and the number of assets exceeds one, the strong sequential core was shown to be generically empty. These results raise the question of whether one can find any conditions to guarantee the non-emptiness of the strong sequential core, apart from trivial cases such as economies with complete sets of state-contingent contracts. In this chapter we derive a set of sufficient conditions in the context of a stationary exchange economy.

A stationary exchange economy is an economy with an infinite time horizon. At each moment of time the economy is in one of a finite number of states. The state determines the consumption sets, the instantaneous utility functions, and the initial endowments of all agents at that moment of time. A transition from one state to the next occurs according to a given Markov process. There are no assets in the economy. A solution to the stationary exchange economy must specify a distribution of the initial endowments, conditional on every finite history of the state of nature.

Assuming that any coalition of players is free to deviate at any moment of time, we consider the subeconomies of the original dynamic economy, where a certain finite history of the state of nature is common knowledge among the players. "The consumption stream is said to be a strong sequential core-element if it belongs to the classical core of every such subeconomy. The main result of the chapter states that the strong sequential core of a stationary exchange economy is non-empty, provided that the discount factor that players apply to future utility streams is sufficiently close to one.

In more detail, the content of the chapter is as follows. In Section 6.2 a stationary

\footnotetext{
${ }^{1}$ This chepter is based on Predtetchinghi, Herings, and Peters [26].
} 
exchange economy is introduced, and the concepts of the classical core and of the strong sequential core are discussed. In Section 6.3 a characterization of the strong sequential core is developed. We show that the classical core and therefore the strong sequential core consist only of stationary, i.e. time and history independent, consumption streams. This property of the core allows for a representation of every dynamic sub-economy by means of a finite-dimensional static evaluation economy. We prove that the strong sequential core is non-empty if and only if cores of the evaluation economies have a non-empty intersection. Section 6.4 presents an example illustrating a non-emptiness criterion for the strong sequential core. Section 6.5 provides a mathematical result that plays a central role in the proof of the non-emptiness of the strong sequential core. This is a convergence theorem for a sequence of matrices that arise when one considers the expected discounted utility of a stationary consumption stream, as the discount factor approaches one. Section 6.6 proves a non-emptiness result for the strong sequential core. More precisely, this result says that a competitive equilibrium allocation of a specific limit economy is in the strong sequential core whenever the discount factor is sufficiently close to one. Section 6.7 concludes.

\subsection{Stationary exchange economies}

A stationary exchange economy is given by the tuple

$$
\mathbf{E}=\left\langle N, \mathbf{S}, L,\left(X_{s}^{i}, u_{s}^{i}, e_{s}^{i}\right)_{s \in \mathbf{S}}^{i \in N}, p, \delta\right\rangle .
$$

The ingredients of a stationary exchange economy $\mathbf{E}$ are as follows. The set, $N=\{1, \ldots, n\}$ is the set of agents and $\mathbf{S}=\{1, \ldots, S\}$ is the set of states. The symbol $L$ denotes the number of consumption goods in each state and each time period. The symbols $X_{s}^{i}, u_{s}^{i}$, and $e_{s}^{i}$ denote, respectively, the consumption set, the instantaneous utility function, and whe vector of initial endowments of agent $i \in N$ in state $s \in \mathrm{S}$. The matrix $p=(p(\sigma \mid s))_{\sigma, s \in \mathrm{S}}$ is th column stochastic matrix of transitional probabilities: for any $\sigma$ and $s$ in $\mathrm{S}, p(\sigma \mid s)$ is thes transition probability from state $s$ to state $\sigma$. We assume that the agents' preferences over consunption streams can be represented by the expected discounted utility function. The discount factor is given by $\delta$.

The basic assumption of the model is the following:

\section{Assumption (A).}

(A1) For all $i \in N$ and $s \in \mathrm{S}$ the consumption set $X$ is a non empty, convex, closed, bounded from below subset of $\mathbb{R}^{L}$.

(A2) For all $i \in N$ and $s \in \mathrm{S}$ the instantaneous utility function $u_{s}^{i}: X_{s}^{i} \rightarrow \mathbb{R}$ is continuous and concave.

(A3) For all $i \in N$ and $s \in \mathbf{S}$, the vector of initial endowments $e_{s}^{i}$ is an element of the consumption set $X_{s}^{i}$. 
(A) The matrix $p$ is irreducible: it is not possible to partition the set $S$ in two subsets $S_{1}$ and $\mathbf{S}_{2}$ in such a way that $p(\sigma \mid s)=0$ for all $s \in \mathbf{S}_{1}$ and all $\sigma \in \mathbf{S}_{2}$.

(A.5) $\delta \in(0,1)$.

A stationary exchange economy is an economy with infinite time horizon, so the set of time periods or dates is $T=\{0,1, \ldots t, \ldots\}$. At each date $t \in T$ the state takes on one of the values from the set $\mathbf{S}$ determining the consumption sets, the instantaneous utility functions, and the initial endownents of all agents at date t. The state follows a Markov process with one-step transitional probabilities given by $p$. At date $t=0$ the state is known to be $s_{0} \in \mathrm{S}$.

The set

$$
\mathbb{D}=\left\{\left(t, s_{0}, s_{1}, \ldots, s_{t}\right) \mid t \in \mathbf{T}, s_{\tau} \in \mathbf{S} \operatorname{for} a n+\in\{1, \ldots t\}\right\}
$$

consists of all possible finite histories of the state of nature. A typical element $\xi=$ $\left(t, s_{0}, s_{1}, \ldots, s_{t}\right)$ of the set $\mathbb{D}$ summarizes the information available to the players at time. t. This information includes the current date $t$ as well as the history $\left(s_{0}, s_{1}, \ldots, s_{p}\right)$ of the state of nature up to the moment $t$. The state of nature $s_{1}$ at the current date $t$ is refened to as a current state. The mapping $t: \mathbb{D} \rightarrow \mathrm{T}$ assigns to each finite history $\xi \in \mathbb{D}$ the current date $t(\xi)$. The mapping $s: \mathbb{D} \rightarrow \mathrm{S}$ assigns to each finite history $\xi$ the current state $s(s)$.

The set of histories D can be thought of as having a structure of the tree. For this reason we shall occasionally refer to a finite history $\xi \in \mathbb{D}$ as a node of the date-event tree. The date event tree is illustrated in Figure 6.1 for the case where the are two states of nature; numbers in bold type indicate the current states.

A given finite history $\xi=\left(t, s_{0}, s_{1}, \ldots, s_{1}\right)$ gives rise to a set of listories that agree with s up to date $t$. Formally, it is defined as

$$
\mathbb{D}(\xi)=\left\{\left(\tau, \sigma_{0}, \sigma_{1}, \ldots, \sigma_{\tau}\right) \in \mathbb{D} \mid \tau \geq t, \sigma_{k}=s_{k} \text { for all } k \in\{0,1, \ldots, t\}\right\} .
$$

The set $\mathbb{D}(\xi)$ can be thought of as a subtree or a branch of the tree D starting at $\xi$. The probability that the state of nature will develop according to $\eta=\left(\tau, \sigma_{0}, \sigma_{1}, \ldots, \sigma_{x}\right)$ conditional on the history $\xi=\left(t, s_{0}, s_{1}, \ldots, s_{n}\right)$ up to date $t$ can be computed using the formulat:

$$
\rho(\eta \mid \xi)= \begin{cases}1, & \text { if } \eta=\xi \\ \prod_{k=k, \|}^{t} p\left(\sigma_{k} \mid \sigma_{k-1}\right), & \text { if } \eta \in \mathbb{D}(\xi) \text { and } t>t \\ 0, & \text { otherwise. }\end{cases}
$$

A mapping $\mathcal{X}^{i}: \mathbb{D} \rightarrow \mathbb{R}^{L}$ is called the consumption stream of agent $i \in N$ if the value $X_{z}^{i}$ at every element $\xi$ of the set $\mathbb{D}$ belongs to the consumption set $X_{\text {wit }}$ ) of agent $i$. The collection of the consumption streams $X=\left\langle X^{\prime}\right\rangle_{\text {ieq }}$ is called a commodity allocation for the coalition of agents $Q \subseteq N$.

The expected discounted utility of the consumption stream $x^{i}$ of player a conditional on the finite history $\xi$ of the state of nature is given by the function

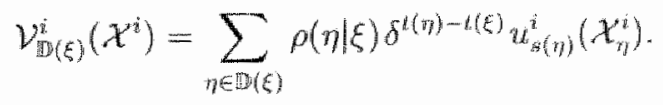




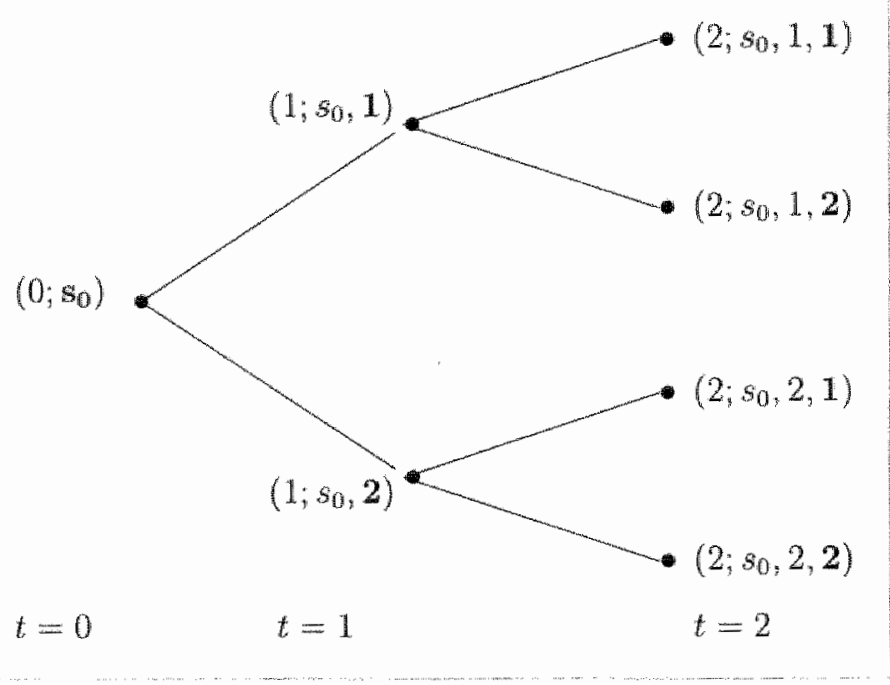

Figure 6.1: The set of finite histories of the state of nature with $S=2$.

Given a stationary exchange economy $\mathbf{E}$ and a finite history $\xi$ of the state of nature. let $\mathbf{E}_{\mathrm{B}(\mathrm{s})}$ denote the subeconomy of $\mathbf{E}$ that follows the realizations of the state of nature as specifed in $\xi$.

We continue with the definitions of the classical core for the subeconomy $\mathbb{E}_{\text {nes }}$ and of the strong sequential core for the economy $\mathbf{E}$. Essentially, the former definition requires that no coalition be able to make all its members better off by deviating at note $\xi$ for the rest of time. The latter definition requires that for no particular history of the state of nature can a coalition of players improve upon a proposed allocation. In this way, the strong sequential core eliminates any incentives for coalitions to first agree to a proposed allocation but breal the agreement later on.

Definition 6.1 A commodity alocation $\mathcal{X}=\left\langle\mathcal{X}^{i}\right\rangle_{\text {ieN }}$ belongs to the classical core $C\left(\mathrm{E}_{\mathrm{m}}(\boldsymbol{\theta})\right.$ of the sub-economy Eres if

1. $\sum_{i \in N} X_{\eta}^{i}=\sum_{i \in N} e_{s(\eta)}^{i}$ for all $\eta \in \mathbb{D}(\xi)$

2. there exist no coalition $Q \subseteq N$ and no commodity allocation $\mathcal{Y}=\left(\mathcal{Y}^{2}\right)_{i \in Q}$ such that

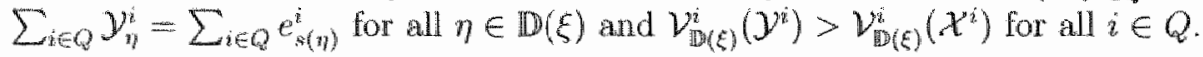

Definition 6.2 The strong sequential core of the stationary exchange conomy $\mathbf{E}$ is the set of commodity allocations

$$
\operatorname{SSC}(\mathbf{E}):=\bigcap_{s \in \mathbb{D}} C\left(\mathbf{E}_{\mathbb{D}(\varepsilon)}\right)
$$




\subsection{A characterization of the strong sequential core}

An important role in the characterization of the strong sequential core is played by stafionary consumption streams. A consumption stream $\mathcal{X}^{i}$ of player $i$ is said to be stationary if the value for $\mathcal{X}_{\xi}^{i}$ depends on the current state $s(\xi)$ only. Thus $\mathcal{X}_{\xi}^{i}$ should be independent of time and of the history of the state of nature preceding the current date. For any stationary consumption stream $X^{i}$, there is an element $x^{i}$ of the set $X^{i}=\underset{\sigma \in S}{\underset{S}{x}} X_{\sigma}^{i}$ such that $X_{\xi}^{i}=x_{s(\xi)}^{i}$ for all $\xi \in \mathbb{D}$; we write $\mathcal{X}^{i}=\left\{x^{i}\right\}$. Let $\{x\}$ denote the stationary commodity allocation $\left\langle\left\{x^{i}\right\}\right\rangle_{i \in N^{*}}$

Now we derive a specific formula for the discounted expected utility of a stationary consumption stream. Let $I_{S}$ denote the $S$-dimensional identity matrix. Observe that the matrix $I_{S}-\delta p$ is invertible, since the spectral radius of the matrix $\delta p$ equals $\delta<1$. Let $\psi=\left(I_{S}-\delta p\right)^{-1}$, and for every pair of states $\sigma$ and $s$ let $\psi(\sigma \mid s)$ denote an element of the matrix $\psi$ at the intersection of row $\sigma$ and column $s$. If the matrix $p$ is irreducible, then $\psi(\sigma \mid s)>0$ for all $\sigma, s \in \mathbf{S}$.

Proposition 6.1 Let $\xi \in \mathbb{D}$ be a fimite history of the state of nature and $x^{i}$ be an element of $X^{i}$. Then

$$
V_{\mathbb{D}(\xi)}^{i}\left(\left\{x^{i}\right\}\right)=\sum_{\sigma \in S} \psi(\sigma \mid s(\xi)) u_{s}^{i}\left(x_{\sigma}^{i}\right)
$$

Proof. Let $\xi \in \mathbb{D}$ be given. First we argue that

$$
\psi(\sigma \mid s(\xi))=\sum_{\substack{\eta \in \mathbb{D}(\xi) \\ s(\eta)=\sigma}} \rho(\eta \mid \xi) \delta^{t(\eta)-t(\xi)} .
$$

Indeed, the right-hand side of Equation (6.2) can be written as

$$
\sum_{\tau \in \mathbf{T}} \delta^{\tau}\left[\sum_{\eta} \rho(\eta \mid \xi)\right]
$$

where the sum in square brackets is taken over all nodes $\eta \in \mathbb{D}(\xi)$ such that $t(\eta)=t(\xi)+T$ and $s(\eta)=\sigma$. The expression in square brackets gives the probability that state $\sigma$ will be reached from state $s(\xi)$ in exactly $\tau$ periods. It is therefore equal to the entry of the matrix $p^{T}$ at the intersection of row $\sigma$ and column $s(\xi)$. Hence, the right-hand side of equation (6.2) equals the entry of the matrix $\sum_{\tau \in \mathrm{T}} \delta^{T} p^{r}$ at the intersection of row $\sigma$ and column $s(\xi)$. Finally, observe that $\sum_{r \in \mathbf{T}} \delta^{\tau} p^{\tau}=\psi$.

Given $x^{i} \in X^{2}$, the expected discounted utility of the consumption stream $\left\{x^{i}\right\}$ conditional on $\xi$ can be written as

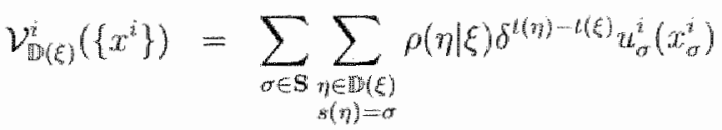

$$
\begin{aligned}
& =\sum_{\sigma \in \mathrm{S}} \psi(\sigma \mid s(\xi)) u_{\sigma}^{i}\left(x_{\sigma}^{i}\right) .
\end{aligned}
$$


Definition 6.3 The evaluation economy $\mathscr{E}_{s}$ in state $s$ is the pure exchange economy where $\mathbb{R}^{S L}$ is the commodity space, $X^{i}=x_{\sigma \in \mathrm{S}} X_{\sigma}^{i}$ is player $i$ 's consumption set, $e^{i}=\left(e_{\sigma}^{i}\right)_{\sigma \in \mathrm{S}}$ is the vector of initial endowments, and $v_{s}^{i}: X^{i} \rightarrow \mathbb{R}$ is the utility function defined by

$$
v_{s}^{i}\left(x^{i}\right)=\sum_{\sigma \in \mathrm{S}} U(\sigma \mid s) u_{\sigma}^{i}\left(x_{\sigma}^{i}\right)
$$

The commodity space of the evaluation economy $\mathscr{E}_{s}$ consists of all stationary consumption streams. As Proposition 6.1 makes it clear, the utility function in $\mathscr{E}_{s}$ is the discounted expected utility of a stationary consumption stream conditional on any finite history of the state of nature where the current state is $s$.

Theorem 6.1 provides a necessary and sufficient condition for a given stationary commodity allocation to be an element of the strong sequential core.

Theorem 6.1 Let $\mathbf{E}$ be a stationary exchange economy satisfying assumption (A). Let $\mathcal{X}=\{x\}$ be a stationary commodity allocation. Then

1. $\mathcal{X} \in C\left(\mathbf{E}_{\mathbb{M}(\xi)}\right)$ if and only if $x \in C\left(\mathscr{E}_{s(\zeta)}\right)$.

2. $X \in S S C(\mathbb{E})$ if and only if $x \in C\left(\mathscr{E}_{s}\right)$ for all $s \in \mathbf{S}$.

Proof. Let the stationary commadity allocation $\mathcal{X}=\{x\}$ be given. To prove the first part of Theorem 6.1 we demonstrate that a coalition $Q \subseteq N$ is able to improve upon $\mathcal{X}$ in the subeconomy $\mathbf{E}_{\mathfrak{D}(\xi)}$ if and only if it can improve upon $x$ in the evaluation economy $\mathscr{E}_{s(\xi)}$. The second part of Theorem 6.1 is an immediate corollary of the first part.

Suppose that there is a deviation $y$ from $x$ by a coalition $Q$ in the evaluation economy $\mathscr{E}_{s(\xi)}$. Proposition 6.1 implies that the stationary commodity allocation $\{y\}$ is a deviation from the allocation $\{x\}$ in the subeconomy $\mathbb{E}_{\mathbb{D}(\xi)}$.

Suppose that there is a deviation $\mathcal{Y}$ from the allocation $\{x\}$ by a coalition $Q$ in the subeconomy $\mathbf{E}_{\mathbb{f y}(\boldsymbol{G})}$. Note that the allocation $\mathcal{Y}$ need not be a stationary allocation. However, as we demonstrate in the remainder of the proof, there does exist a stationary allocation $\mathcal{Z}=\{z\}$ that is feasible for $Q$ and gives each member of the coalition $Q$ at least as high expected discounted utility conditional on $\xi$ as $\mathcal{Y}$ does. An allocation $\mathcal{Z}$ having these properties is a deviation from the allocation $\{x\}$ in the subeconomy $\mathbf{E}_{\mathbb{Q}}(\xi)$. By Proposition $6.1, z$ is a deviation from $x$ in the evaluation economy $\mathscr{E}_{s(\xi)}$.

Define $z$ by

$$
z_{s}^{i}=\frac{1}{\psi(\sigma \mid s(\xi))} \sum_{\substack{\eta \in \mathbb{D}(\xi) \\ s(\eta)=\sigma}} \rho(\eta \mid \xi) \delta^{t(\eta)-t(\xi)} \mathcal{Y}_{\eta}^{i}
$$

for all $i \in Q$ and $\sigma \in \mathbf{S}$. As the allocation $\mathcal{Y}$ is feasible for coalition $Q$, and the consumption sets $X_{\sigma}^{i}$ are assumed to be bounded from below for all $i \in Q$, the consumption stream $\mathcal{Y}^{i}$ is bounded. Hence, the sum in Equation (6.3) is well-defined. Equation (6.2) implies that the coefficients in Equation (6.3) add up to one, so that $z_{\sigma}^{i}$ is a convex combination of the values for the consumption stream $y^{i}$ at all nodes $\eta$ in the subtree $\mathbb{D}(\xi)$ with $s(\eta)=\sigma$. 
For all such nodes, $\mathcal{Y}_{\eta}^{i} \in X_{v}^{i}$. Since the set $X_{\sigma}^{i}$ is assumed to be closed and convex, $z_{\sigma}^{i} \in X_{\sigma}^{i}$. Moreover, $z$ is feasible for $Q$. Continuity and concavity of the instantaneous utility functions imply the inequalities

$$
u_{\sigma}^{i}\left(z_{\sigma}^{i}\right) \geq \frac{1}{\psi(\sigma \mid s(\xi))} \sum_{\substack{\eta \in D(\xi) \\ s(\eta)=\sigma}} \rho(\eta \mid \xi) \delta^{t(\eta)-t(\xi)} u_{o}^{i}\left(Y_{\eta}^{i}\right),
$$

for all $i \in Q, \sigma \in \mathrm{S}$. By Proposition 6.1, the discounted expected utility of the consumption stream $Z^{i}=\left\{z^{i}\right\}$ is

$$
\begin{aligned}
& \mathcal{V}_{\mathbb{D}(\xi)}^{i}\left(\mathcal{Z}^{i}\right)=\sum_{\sigma \in \mathrm{S}} \psi(\sigma \mid s(\xi)) u_{\sigma}^{i}\left(z_{\sigma}^{i}\right) \\
& \geq \sum_{\sigma \in \mathbb{S}} \sum_{\substack{\eta \in \mathbb{D}(\xi) \\
s(\eta)=\sigma}} \rho(\eta \mid \xi) \delta^{t(\eta)-t(\xi)} u_{\sigma}^{i}\left(\mathcal{Y}_{\eta \eta}^{i}\right) \\
& =\sum_{\eta \in \mathbb{D}(\xi)} \rho(\eta \mid \xi) \delta^{\ell(\eta)-r(\xi)} u_{s(\eta)}^{i}\left(\mathcal{Y}_{r_{i}}^{i}\right) \\
& =\mathcal{V}_{i p(\xi)}^{i}\left(\mathcal{Y}^{i}\right)
\end{aligned}
$$

for all $i \in Q$.

Theorem 6.2 gives sufficient conditions for the classical core to contain only stationary commodity allocations.

Theorem 6.2 Let $\mathbf{E}$ be a stationary exchange economy satisfying assumption (A) in which $p(\sigma \mid s)>0$ for all $\sigma, s \in \mathbf{S}, X_{\sigma}^{i}=\mathbb{R}_{+}^{L}, u_{\sigma}^{i}$ are strongly monotone ${ }^{2}$ for all $i \in N, \sigma \in \mathbf{S}$ and strictly concave for all but possibly one player in $N$ and for all states $\sigma \in \mathbf{S}$. Then all commodity allocations in the classical core of the economy $\mathbf{E}$ are stationary.

Proof. Let $\mathcal{X}$ be an $N$-feasible commodity allocation. If the allocation $\mathcal{X}$ is not stationary, then there exists a pair of nodes $\lambda, \varsigma \in \mathbb{D}$ with the same current state $\sigma$ such that, $\mathcal{X}_{\lambda}^{i_{4}} \neq \mathcal{X}_{c}^{i_{i}}$ for some $i_{0} \in N$. Observe that both $\mathcal{X}_{\lambda}^{i}$ and $\mathcal{X}_{\xi}^{i}$ are elements of the consumption set $X_{j}^{i}$ for all $i \in N$.

Let $\rho(\lambda)$ and $\rho(\varsigma)$ be probabilities to reach nodes $\lambda$ and $\varsigma$ conditional on the initial node $\left(0, s_{0}\right)$. Under the assumption that all transition probabilities are positive, $\rho(\lambda)$ and $\rho(s)$ are both positive. Define an element $\widehat{y}_{\sigma}^{i}$ of the set $X_{o}^{i}$ as

$$
\bar{y}_{\sigma}^{i}=\frac{\delta^{\ell(\lambda)} \rho(\lambda) \mathcal{X}_{\lambda}^{i}+\delta^{l(\zeta)} \rho(\varsigma) \mathcal{X}_{\zeta}^{i}}{\delta^{t(\lambda)} \rho(\lambda)+\delta^{t(\zeta)} \rho(\varsigma)}
$$

The function $u_{\sigma}^{i}$ is strongly monotone if the following condition is satisfind: $\int x_{\sigma}^{i}, y_{\alpha}^{i} \in\left[R_{-}^{L}, x_{\sigma l}^{i} \geq\right.$ $\left.y_{\sigma x l}^{i} \forall l \in\{1, \ldots, L\}, x_{\sigma}^{i} \neq y_{\sigma}^{i}\right]$ implies $\left[u_{\sigma}^{i}\left(x_{\sigma}^{i}\right)>u_{\sigma}^{i}\left(y_{\sigma}^{i}\right)\right]$. 
By concavity of the instantaneous utility functions,

$$
u_{\sigma}^{i}\left(\widetilde{y}_{\sigma}^{i}\right) \geq \frac{\delta^{(i \lambda)} \rho(\lambda) u_{\sigma}^{i}\left(\mathcal{X}_{\lambda}^{i}\right)+\delta^{((\varsigma)} \rho(\varsigma) u_{\sigma}^{i}\left(\mathcal{X}_{\varsigma}^{i}\right)}{\delta^{i(\lambda)} \rho(\lambda)+\delta^{i(\varsigma)} \rho(\varsigma)}
$$

for all $i \in N$.

Since $\sum_{i \in N} \mathcal{X}_{v_{i}}^{i_{i}}=\sum_{i \in N} \mathcal{X}_{\zeta}^{i}$, there must be $i_{1} \in N \backslash\left\{i_{0}\right\}$ such that $\mathcal{X}_{\lambda}^{i_{1}} \neq \mathcal{X}_{\varsigma}^{i_{3}}$. As the requirement of strict concavity is violated for at most one agent, the utility function $u_{\sigma}^{:}$ must be strictly concave either for $i=i_{0}$ or for $i=i_{1}$. Thus, inequality (6.4) is strict for at least one agent in the economy. Hence, an $N$-feasible allocation $y_{\sigma}$ can be constructed in such a way that, when it is substituted for $\widetilde{y}_{\sigma}$ in (6.4), the inequalities become strict for all $i \in N{ }^{3}$

Define the consumption stream $\mathcal{Y}^{i}$ of agent $i$ as follows:

$$
\mathcal{Y}_{\eta}^{i}= \begin{cases}\mathcal{X}_{\eta}^{i}, & \text { if } \eta \in \mathbb{D} \backslash\{\lambda, \varsigma\} \\ y_{\sigma}^{i}, & \text { if } \eta=\lambda, \text { or } \eta=\varsigma .\end{cases}
$$

Then the discounted expected utility of the consumption stream $\mathcal{Y}^{i}$ at the initial node is greater than that of the consumption stream $\mathcal{X}^{i}$ for every member $i$ of coalition $N$. Hence, allocation $\mathcal{Y}$ is a utility improving deviation by coalition $N$ from allocation $\mathcal{X}$.

We conclude this section with a characterization of the strong sequential core.

Corollary 6.1 Let $\mathbf{E}$ be a stationary exchange economy satisfying all assumptions of Theorem 6.2. Then

$$
S S C(\mathbf{E})=\left\{\{x\} \mid x \in \bigcap_{s \in \mathrm{S}} C\left(\mathscr{E}_{s}\right)\right\} .
$$

In the special case with only one state of nature the strong sequential core $S S C(\mathbf{E})$ coincides with the classical core $C(\mathbb{E})$. Both the strong sequential core and the classical core consist of those consumption allocations where some distribution of initial endowments $x$ is repeated each period of time, with $x$ being a core element of the evaluation economy $\mathscr{E}_{1}$. Thus $S S C(\mathbf{E})$ is non-empty. As Corollary 7.1 indicates, however, if there are at least two states of nature, and if the cores of the evaluation economies in different states are disjoint, then the strong sequential core is empty. The following example illustrates the point.

The allocation $y_{\sigma}$ can be constructed als follows. Suppose that inequality (6.4) is strict for agent $i_{0}$.

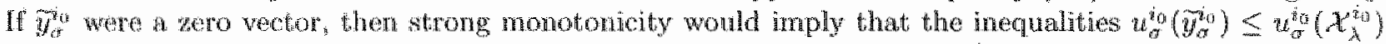

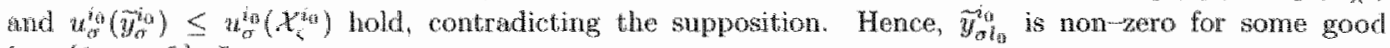
$l_{0} E\{1, \ldots, L\}$. Let

$$
y_{a l}^{i}= \begin{cases}\breve{y}_{c l}^{i}, & \text { if } l \in\{1, \ldots, L\} \backslash\left\{l_{0}\right\}, i \in N, \\ \breve{y}_{o l}^{i}-\epsilon & \text { if } l=l_{03}, i=i_{0} \\ \vec{y}_{a l}^{i}+\frac{1}{n-1} \epsilon & \text { if } l=l_{0}, i \in N \backslash\left\{i_{0}\right\} .\end{cases}
$$

Then by strong monotonicity, $u_{\sigma}^{i}\left(y_{\sigma}^{i}\right)>u_{\sigma}^{i}\left(\bar{y}_{\sigma}\right)$ for all $\epsilon>0$ and all $i \in N \backslash\left\{i_{0}\right\}$. Moreover, $c^{\prime}>0$ can be chosen small enongh for the vector $y_{\sigma}^{i_{0}}$ to lie in the consumption set. $X_{\sigma}^{i}$ and to preserve strict inequality in (6.4). 


\subsection{An example}

Consider a stationary exchange economy with two agents called $a$ and $b$, two states $(S=2)$, and a single consumption good in each state $(L=1)$. The instantaneous utility functions are

$$
u_{s}^{i}\left(x_{s}^{i}\right)=\ln \left(x_{s}^{i}\right), i=a, b
$$

For a moment, we leave the matrix of transitional probabilities and the initial endowments unspecified. Aggregate endowment of the consumption good in state $\sigma$ is denoted by $e_{\sigma}^{M}$, and $e^{N} \in \mathbb{R}^{S}$ is the vector of aggregate endowments across all states.

The set of Pareto-efficient allocations of the evaluation economy coincides with the diagonal of the Edgeworth box. That is, distribution $x$ of the initial endownents is Paretoefficient if agents' consumption bundles $x^{i}$ are proportional to $e^{N}$. For a Pareto-efficient allocation to be in the core of the evaluation economy, the share of the aggregate endowment accrued to each agent should be large enough, so that the conditions of individual rationality hold. Formaily,

$$
C\left(\mathscr{G}_{s}\right)=\left\{\left(x^{a}, x^{b}\right) \in \mathbb{R}^{S} \times \mathbb{R}^{s} \mid \exists t^{a}, t^{b}>0: \begin{array}{c}
t^{i}+t^{b}=1 \\
t^{i} \geq t^{i}, i=a, b \\
t_{s}^{i}, i=a, b
\end{array}\right\}
$$

The vallue for $t_{s}^{i}$ is determined by the condition of individual rationality for agent $i$. It depends on the fundamentals of the stationary exchange economy as follows:

$$
\underline{t_{s}^{i}}=\prod_{\sigma \in \mathrm{S}}\left(\frac{e_{\sigma}^{i}}{e_{\sigma}^{N}}\right)^{(1-\delta) \phi^{i}(\sigma|\sigma| s)} .
$$

Any allocation in the core of the evaluation economy is wholly determined by $t^{a}$, the share of the aggregate endowment enjoyed by agent $a$. In stats $s$ the lower value for $t^{\text {ax }}$ is $t_{g}$. while the upper value is $1-t_{s}^{b}$. We know (see Corollary 7.1) that the strong sequential $\overrightarrow{c o r e}$ is non-empty if and only if the cores of the evaluation economies in different states have a non-empty intersection. In our example, this is equivalent to the condition that the intervals $\left[\underline{t_{1}^{a}}, 1-t_{1}^{t_{0}}\right]$ and $\left[t_{2}^{a}, 1-t_{2}^{b}\right]$ overlap.

Figure 6.2 pictures these intervals for various values of the discount factor $\delta$. To produce this figure the transitional probabilities and the initial endowments were specified as follows:

$$
p=\left[\begin{array}{ll}
0.75 & 0.25 \\
0.25 & 0.75
\end{array}\right], e^{a}=\left[\begin{array}{l}
4 \\
1
\end{array}\right], e^{b}=\left[\begin{array}{l}
1 \\
4
\end{array}\right] .
$$

We see that for the values of $\delta$ smailer than, approximately, 0.8 , the cores of the evaluation economies are disjoint, and consequently the strong sequential core is empty. In contrast, for $\delta$ greater than 0.8 the strong sequential core is non-empty. The example therefore suggests that the more patient individnals are, the more likely it is that the strong sequential core is non-empty. Section 6.6 validates this suggestion for the general case. 


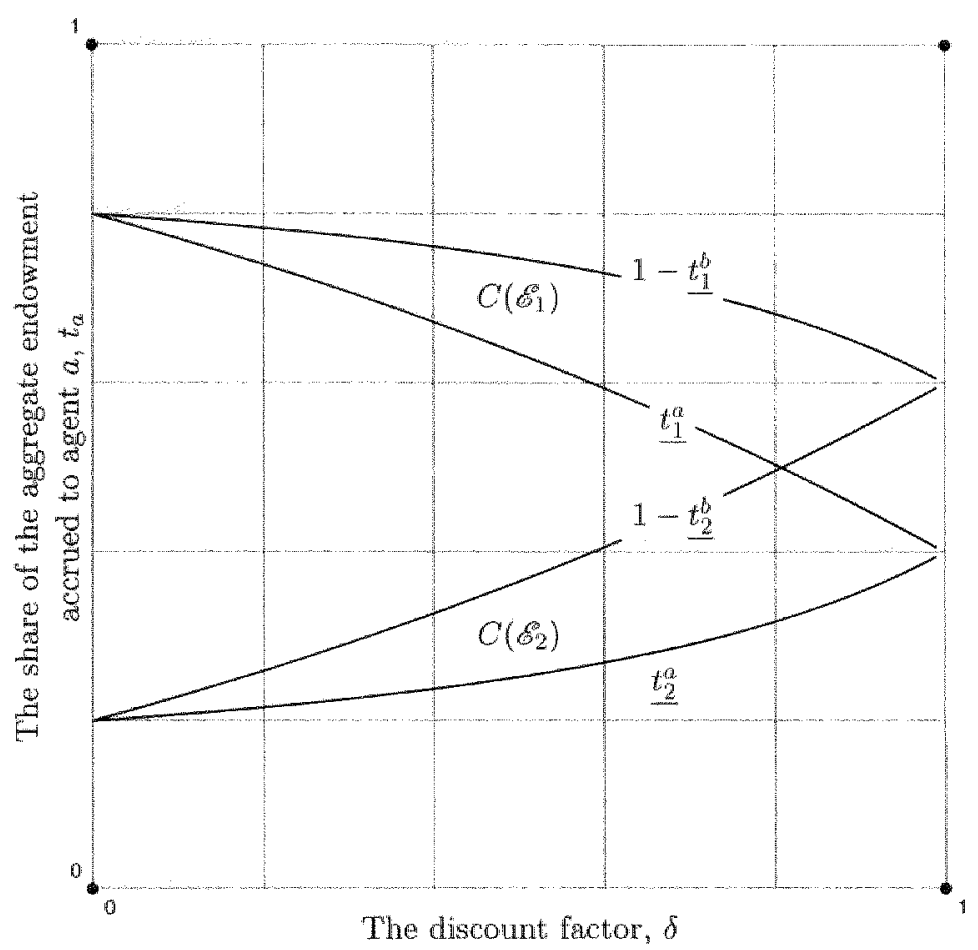

Figure 6.2: The cores of the evaluation economies 


\subsection{A convergence theorem}

In this section we prove a convergence theorem that plays a key role in the analysis of the asymptotic behavior of the strong sequential core.

First recall that, for a given column stochastic $(S \times S)$-dimensional matrix $p$, the stationary distribution $\phi$ is a probability distribution over the set $\mathbf{S}$ satisfying the equation $\mathbb{p} \phi=\phi$. Here we are regarding $\phi$ as a column $S$-dimensional vector. Since the state space $\mathbf{S}$ is finite by assumption, there is at least one stationary distribution. If the matrix $p$ is irreducible, then there is a unique stationary distribution $\phi$. Moreover, $\phi(s)>0$ for all $s \in \mathbf{S}$.

Theorem 6.3 Let $p$ be a column stochastic irreducible $(S \times S)$-dimensional matrix. Let $\phi=(\phi(1)), \ldots, \phi(S))^{\top}$ be the stationary distribution over the set $\mathrm{S}$. Then

$$
\lim _{\substack{\delta<1 \\
0<\delta<1}}(1-\delta)\left(I_{S}-\delta p\right)^{-1}=\left(\begin{array}{ccc}
\phi(1) & \cdots & \phi(1) \\
\vdots & & \vdots \\
\phi(S) & \cdots & \phi(S)
\end{array}\right) .
$$

Proof. Let $\lambda_{1}, \ldots, \lambda_{m}$, where $m \leq S$, be the distinct eigenvalues of the matrix $p$; lec $\lambda_{1}=1$. Let $p=T \Lambda T^{-1}$ be the Jordan decomposition of the matrix $p$, where $\Lambda$ is a Jordan form.

It is well-known that $\lambda_{1}$ is an algebraically (and hence geometrically) simple eigenvalue of the matrix $p$. Therefore, there is a unique Jordan block corresponding to the eigenvalue $\lambda_{1}$ and this block has order 1 . Hence, the Jordan form $\Lambda$ can be written as

$$
\Lambda=\left(\begin{array}{ll}
1 & 0 \\
0 & \Gamma
\end{array}\right)
$$

where $\Gamma$ is a direct sum of those Jordan blocks that correspond to the eigenvalues $\lambda_{2}, \ldots, \lambda_{n}$. Since $\Gamma$ is an upper triangular matrix whose diagonal elements are $\lambda_{2}, \ldots, \lambda_{v n}$, the matrix $\left(I_{S-1}-\delta \Gamma\right)$ is non-singular whenever $\delta \neq \frac{1}{\lambda_{j}}$ for $j=2, \ldots, m$. In particular, the matrix $\left(I_{S-1}-\Gamma\right)$ is non-singular.

Now for all values of $\delta$ sufficiently close to one, we can write

$$
\left(I_{S}-\delta p\right)^{-1}=T\left(I_{S}-\delta \Lambda\right)^{-11} T^{-1},
$$

and

$$
\left(I_{S}-\delta \Lambda\right)^{-1}=\left(\begin{array}{cc}
(1-\delta)^{-1} & 0 \\
0 & \left(I_{S-1}-\delta \Gamma\right)^{-1}
\end{array}\right)
$$

As $\delta$ approaches one, the matrix $\left(I_{S-1}-\delta \Gamma\right)^{-1}$ converges to $\left(I_{S-1}-\Gamma\right)^{-1}$. Therefore,

$$
\lim _{\substack{\delta \rightarrow 1 \\ 0<\hat{\delta}<1}}(1-\delta)\left(I_{S}-\delta \Lambda\right)^{-1}=\operatorname{diag}(1,0, \ldots, 0),
$$


and

$$
\lim _{\substack{\delta \rightarrow \infty \\ 0<i<1}}(1-\delta)\left(I_{S}-\delta p\right)^{-1}=T \operatorname{diag}(1,0, \ldots, 0) T^{-1}=\tau p,
$$

where $T$ is the first column of the matrix $T$, and $\rho$ is the first row of the matrix $T^{-1}$.

As $p T^{\prime}=T \Lambda$, we see that $p \tau=\tau$. From $T^{-1} p=\Lambda T^{-1}$ it follows that $p p=\rho$. That is, $\rho^{\top}$ is the eigenvector of the matrix $p^{\top}$ corresponding to the eigenvalue $\lambda_{1}=1$. Therefore, $\rho$ is proportional to the vector $\mathbf{1}_{s}=(1, \ldots, 1)$, so we can write $\rho=\left(\rho_{1}, \ldots, \rho_{1}\right)$.

Setting $\phi=\rho_{1} \tau$, we see that Equation (7.5) holds, and that $p \phi=\phi$. It remains to show that the entries of the vector $\phi$ add up to one. This immediately follows from the fact that the entries of each column of the matrix $(1-\delta)\left(I_{S}-\delta p\right)^{-1}$ add up to one.

\subsection{A non-emptiness result}

In this section we consider a family of stationary exchange economies $\mathbf{E}_{\delta}$ parameterized by a discount factor $\delta \in(0,1)$. We shall prove that the strong sequential core of the economy $\mathrm{E}_{\delta}$ is non-empty whenever $\delta$ is sufficiently close to one.

To do so, we construct a finite-dimensional economy similar to the evaluation economies, where the stationary distribution $\phi$ over the states of nature is employed to define the expected utility functions. Then we clemonstrate that all Walrasian equilibria of this artificial economy are contained in the strong sequential core, whenever $\delta$ is close enough to one.

Definition 6.4 The economy $\mathscr{E}_{\infty}$ is a pure exchange economy where $\mathbb{R}^{S L}$ is the commodity space, $X^{i}=\times_{\sigma \in \mathrm{S}} X_{\sigma}^{i}$ is the consumption set, $e^{i}=\left(e_{\sigma}^{i}\right)_{\alpha \in \mathrm{S}}$ is the vector of initial cndowments, and $\vartheta^{i}: X^{i} \rightarrow \mathbb{R}$ is the utility function defined by

$$
g^{i}\left(x^{i}\right)=\sum_{\sigma \in \mathbf{S}} \phi(\sigma) u_{o s}^{i}\left(x_{o}^{i}\right)
$$

Theorem 6.4 Let $\mathbf{D}_{\delta}$ be a family of stationary exchange economies, satisfying assumption (A), in which the instantaneous utility functions $u_{s}^{i}$ are strictly concave and locally nonsatiated for all $i \in N$, and $s \in \mathrm{S}$. Let $\vec{x}$ be an equilibrium allocation of the economy $\mathscr{E}_{\infty}$. Then there exists a $\bar{\delta} \in(0,1)$ such that the stationary commodity allocation $\{\bar{x}\}$ belongs to the strong sequential core of the stationary exchange economy $\mathbf{E}_{\delta}$ whenever $\bar{\delta}<\delta<1$.

Proof. Let a vector $\rho=(\rho(1), \ldots, \rho(S)) \in \mathbb{R}_{+++}^{S}$ and a coalition $Q \subseteq N$ be given. Let $\sigma_{Q p}$ be a pure exchange economy where $Q$ is a set of players, $\mathbb{R}^{S L}$ is the commodity space, $X^{i}=x_{\sigma \in \mathrm{S}} X_{\alpha}^{i}$ is the consumption set, $e^{i}=\left(e_{\sigma}^{i}\right)_{\sigma \in \mathrm{S}}$ is the vector of initial endowments, and $v_{\rho}^{i}: X^{i} \rightarrow \mathbb{R}$ is the utility function defined by

$$
v_{p}^{i}\left(x^{i}\right)=\sum_{\sigma \in \mathrm{S}} \rho(\sigma) u_{\sigma}^{i}\left(x_{\sigma}^{i}\right)
$$


Clearly, the evaluation economies $\mathscr{E}_{s}$ and the economy $\mathscr{E}_{\infty}$ are the special cases of the economy $\mathscr{E}_{N_{p}}$, corresponding to $\rho=(\psi(1 \mid s), \ldots, \psi(S \mid s))$ and $\rho=\phi$, respectively.

Let $X_{\sigma}(Q)$ be the set of $Q$-feasible allocations in state $\sigma$, and let $X(Q)$ be the set of $Q$-feasible state-contingent allocations. That is,

$$
\begin{gathered}
X_{\sigma}(Q)=\left\{x_{\sigma} \in \underset{i \in Q}{\times} X_{\sigma}^{i} \mid \sum_{i \in Q} x_{\sigma}^{i}=\sum_{i \in Q} e_{\sigma}^{i}\right\}, \\
X(Q)=\underset{\sigma \in \mathrm{S}}{\times} X_{\sigma}(Q) .
\end{gathered}
$$

Recall that a state-contingent allocation $x \in X(Q)$ is weakly Pareto-efficient in the economy $\mathscr{E}_{Q \rho}$ if there is no $y \in X(Q)$ such that $v_{\rho}^{i}\left(y^{i}\right)>v_{\rho}^{i}\left(x^{i}\right)$ for all $i \in Q$. As a first step towards the proof of Theorem 6.4 , we argue that for any two vectors $\rho, \eta \in \mathbb{R}_{++}^{S}$ the set of weakly Pareto-efficient allocations of the economy $\mathscr{E}_{Q p}$ coincides with that of the oconomy $\mathfrak{B}_{\mathbb{Q}}$

It is well-known that an allocation $x$ is weakly Pareto efficient in $\mathscr{E}_{Q p}$ if and only if there exist non-negative reals $\alpha^{i}$ adding up to one such that $x$ maximjzes the function

$$
\sum_{i \in Q} \alpha^{i} v_{\rho}^{i}\left(x^{i}\right)=\sum_{\sigma \in \mathrm{S}} \rho(\sigma) \sum_{i \in Q} \alpha^{i} u_{\sigma}^{i}\left(x_{\sigma}^{i}\right)
$$

over the set $X(Q)$. Since the coefficients $\rho(s)$ are positive, this is equivalent to the requirement that $x_{\sigma}$ maximizes the function.

$$
\sum_{i \in Q} \alpha^{i} u_{\sigma}^{i}\left(x_{\sigma}^{i}\right)
$$

over $X_{\sigma}(Q)$ for all $\sigma \in \mathrm{S}$. This in its is equivalent to the condition that $x$ be a maximizer of the function

$$
\sum_{\sigma \in \mathrm{S}} \eta(\sigma) \sum_{i \in Q} \alpha^{i} w_{\sigma}^{i}\left(x_{\sigma}^{i}\right)=\sum_{i \in Q} \alpha^{i} v_{\eta}^{i}\left(x^{i}\right)
$$

over the set $X(Q)$. This means that $x$ is weakly Pareto efficient in the economy $\mathscr{E}_{Q n}$.

The utility functions in the evaluation economies depend on the discount factor via $\psi$ :

$$
v_{\delta s}\left(x^{i}\right)=\sum_{\sigma \in \mathrm{S}} \psi_{\delta}(\sigma \mid s) u_{\sigma r}^{i}\left(x_{\sigma}^{i}\right)
$$

Theorem 6.3 applies to show that for all $s \in \mathbf{S}$

$$
\lim _{\substack{\delta \rightarrow 1 \\ 0<\delta<1}}(1-\delta) v_{\delta s}\left(x^{i}\right)=\vartheta^{i}\left(x^{i}\right) \text {. }
$$

Now we prove Theorem 6.4. We have to show that for all values of $\delta$ sufficiently close to one and for all states $s \in \mathrm{S}$, the allocation $\vec{x}$ is in the core of the evaluation economy $\mathcal{B}_{6 s}$. Suppose that this is not the case. Then there exist a state $s \in \mathrm{S}$, coalition $Q \subseteq N$, 
and the sequence of discount factors $\delta^{q} \in(0,1)$ converging to 1 such that the coalition $Q$ is able to improve upon $\bar{x}$ in the evaluation economy $\mathscr{E}_{\delta^{q}}$. Let $x^{q}$ be a sequence of deviations, i.e. the sequence of allocations in $X(Q)$ satisfying the inequalities

$$
v_{\delta^{4} \psi_{s}}^{i}\left(x^{q i}\right)>v_{\delta \psi_{s}}^{i}\left(\bar{x}^{i}\right) \text { for all } i \in Q \text {. }
$$

Since $X(Q)$ is a compact set, we can assume without loss of generality that $x^{q}$ converges to an element $x$ of $X(Q)$. Multiplying Inequalities (6.6) by $\left(1-\delta^{q}\right)$ and taking the limit yields the inequalities

$$
\vartheta^{i}\left(x^{i}\right) \geq \mathscr{V}^{i}\left(x^{i}\right) \text { for all } i \in Q \text {. }
$$

Let $\vec{p}$ be a vector of prices corresponding to the equilibrium allocation $\bar{x}$. Under the assumption of local non-satiation of the instantaneous utility functions, Inequalities (6.7) imply that

$$
\bar{p} x^{i} \geq \bar{p} e^{i} \text { for all } i \in Q .
$$

All of these inequalities should be binding, otherwise $x$ would not be feasible for coalition Q. Thus,

$$
\vec{p} x^{i}=\bar{p} e^{i} \text { for all } i \in Q .
$$

The strict concavity assumption implies that there exists a unique maximizer of the utility function $\vartheta^{i}$ in the budget set at prices $\bar{p}$. Therefore,

$$
x^{i}=\bar{x}^{i} \text { for all } i \in Q \text {. }
$$

It follows that the allocation $\left(\bar{x}^{i}\right)_{i \in Q}$ is an equilibrium allocation, and is therefore weakly Pareto efficient in the economy $\mathscr{E}_{\infty}$ restricted to coalition $Q$. We have already proved, however, that any weakly Pareto efficient allocations in the economy $\mathscr{E}_{\infty}$ restricted to $Q$ is also Pareto efficient in the economy $f_{s, s}^{\infty}$ restricted to the same coalition, for any state $s$ atind any value of the discount factor $\delta \in(0,1)$. This leads to a contradiction with Inequalities (6.6).

It remains to give explicit assumptions that will guarantee existence of an equilibrium for the economy $\mathscr{E}_{\infty}$. We require that the instantaneous utility functions be strongly monotone and that the aggregate endowments of commodities be positive.

Corollary 6.2 Let $\mathbf{E}_{\delta}$ be a family of stationary exchange economies, satisfying assumption (A), in which the instantaneous utility functions $u_{s}^{i}$ are strictly concave and strongly monotone for all $i \in N$ and $s \in \mathbf{S}$, and $\sum_{i \in N} e_{s l}^{i}>0$ for all $l \in\{1, \ldots, L\}$ and $s \in \mathbf{S}$. Then there exists a $\vec{\delta} \in(0,1)$ such that the strong sequential core of the economy $\mathbf{E}_{\dot{\delta}}$ is non-empty whenever $\bar{\delta}<\delta<1$.

An example below illustrates that a violation of the strict concavity assumption in Corollary 6.2 may result in the strong sequential core being empty for all values of the discount factor. 
Example 6.1 Consider a family $\mathbf{E}_{\delta}$ of stationary exchange economies with three agents called $a, b$, and $c$, two states $(S=2)$, and a single consumption good in each state $(L=1)$. The state-independent instantaneous utility functions are given by

$$
\begin{aligned}
& u_{\sigma}^{i}\left(x_{\sigma}^{i}\right)=\ln \left(x_{\sigma}^{i}\right), \quad x_{\sigma}^{i}>0, \quad i=a, b, \\
& u_{\sigma}^{c}\left(x_{\sigma}^{i}\right)=x_{\sigma}^{c}, x_{\sigma}^{c} \geq 0 .
\end{aligned}
$$

Notice that the function $u_{o}^{c}$ is not strictly concave, thus violating one of the assumptions of Corollary 6.2. The initial endowments and the transition probabilities are specified as follows:

$$
e^{a}=\left[\begin{array}{l}
4 \\
1
\end{array}\right], e^{b}=\left[\begin{array}{l}
1 \\
4
\end{array}\right], e^{c}=\left[\begin{array}{l}
5 \\
5
\end{array}\right], p=\left[\begin{array}{ll}
0.25 & 0.25 \\
0.75 & 0.75
\end{array}\right]
$$

The core of the evaluation economy is then a single-element seto given by

$$
C\left(\mathscr{E}_{s, \delta}\right)=\left\{\left[\begin{array}{ccc}
\kappa_{s \delta}^{a} & \kappa_{s \delta}^{b} & 5 \\
\kappa_{s \delta}^{a} & \kappa_{s \delta}^{b} & 5
\end{array}\right]\right\}
$$

where the values for $\kappa_{s \dot{b}}^{i}$ depend on the fundamentals of the economy $\mathbf{E}_{\delta}$ as follows:

$$
\kappa_{s \delta}^{i}=(1-\delta) \sum_{\sigma \in \mathrm{S}} \psi_{\delta}(\sigma \mid s) e_{\sigma}^{i}
$$

It is easy to check that $\kappa_{1 \delta}^{a} \neq \kappa_{2 \delta}^{a}$, implying that $C\left(\mathscr{E}_{1 \delta}^{a}\right) \Gamma C\left(\mathscr{E}_{2 \delta}\right)=\varnothing$ for all $\delta \in(0,1)$. Observe that the economies $\mathbf{E}_{\hat{8}}$ satisfy all assumptions of Theorem 6.2. Corollary 7.1 therefore implies that the strong sequential core of the economy $\mathbf{E}_{\delta}$ is empty for all $\delta \in$ $(0,1)$.

\subsection{Concluding remarks}

We consider an economy with infinite time horizon where at each moment of time the state reveals and determines the instantaneous utility functions and initial endowments at that moment. The state follows a Markov process. Contrary to the stochastic-games literature we assume that the transitional probabilities are entirely exogenous in the sense that they camot be infuenced by the agent's consumption decisions. This makes a sub-oconomy starting at a particular point in time independent of the commodity allocation chosen up to that point. Thus the dynamic links between the time periods are rather limited.

While this may seem a restrictive assumption, it is not obvious exactly how the decisions of the players or coalitions should influence the transitional probabilities, given the cooperative nature of the economy in question. Neither can the agents influence futrure sub-economies by trading assets. Indeed, the assumption of the model is that contracts allowing for intertemporal trade are not enforceable. This should be seen as an extreme case of market incompleteness. As we have in Chapter 4, however, the strong sequential core is weakly increasing in the number of enforceable securities. Thus, for the purpose of obtaining the non-emptiness result, the case where there are no securities is the most difficult one. 



\section{Chapter 7}

\section{The Strong Sequential Core in a Stationary Cooperative Game}

\subsection{Introduction}

The analysis of the strong sequential core carried out in this chapter is motivated by the findings of Chapter 6. We translate the non-emptiness result obtained in the framework of a stationary exchange economy to a more general class of cooperative games.

We study a cooperative game with infinite time horizon. At each moment in time the game is in one of a finite number of states. The state determines the instantaneous non-transferable utility game to be played at that moment of time. Transition from one state to the next occurs according to an exogenously given Markov process. A solution to a stationary cooperative game must specify the payoffs to each player, contingent on every finite history of the state of nature. The utility stream is said to be an element of the strong sequential core if it belongs to the classical core of every subgame of the original stationary cooperative game.

The main result of the chapter states that the strong sequential core in a stationary cooperative game is non-empty, provided that

(i) the instantaneous NTU-games in all states satisfy an additive $\pi$-balancedness condition,

(ii) at least one of these NTU-games satisfies a strong additive $\pi$-balancedness condition, and

(ii) the discount faction is sufficiently close to one.

Both additive $\pi$-balancedness and strong additive $\pi$-balancedness strengthen Billera's $\pi$ balancedness condition (see Billera $[4]$. A special form of additive $\pi$-balancedness condition was used earlier by Billera and Bixby to obtain a characterization of market games (see, for example, [6] and [5]). 
This result does not imply our earlier theorem establishing the non-emptiness of the strong sequential core in a stationary exchange economy with a sufficiently high discount factor. Indeed, the instantaneous NTU-games constructed using the data of the stationary exchange economy are always additively $\pi$-balanced, but may well violate the strong additive $\pi$-balancedness condition.

The outline of the chapter is as follows. In Section 7.2 stationary cooperative games are presented and the assumptions of the model are discussed. In Section 7.3 a characterization of the strong sequential core is developed, in line with the analysis presented in Chapter 6. In particular, we demonstrate that the classical core of the stationary cooperative game consists only of stationary utility streams, provided that all transition probabilities are positive and feasibility sets are strictly convex. We prove that the strong sequential core is non-empty if and only if the cores of the evaluation games have a non-empty intersection. The additive balancedness and strong additive balancedness conditions are discussed in Section 7.4. Existence results are reported in Section 7.5. We prove that any utility allocation in the proper core of a specific limit game belongs to the strong sequential core whenever the discount factor is close to one. An example is presented in Section 7.6.

\subsection{Stationary cooperative games}

A stationary cooperative game is given by the tuple

$$
\Gamma=\left\langle N, \mathrm{~S},\left(U_{s}\right)_{s \in \mathrm{S}}, p, \delta\right\rangle .
$$

The ingredients of a stationary cooperative game $\Gamma$ are as follows. The set $N$ is the set of players and $\mathbf{S}=\{1, \ldots, S\}$ is the set of states. The symbol $U_{s}$ denotes the instantaneous non-transferable utility game $\left\langle U_{s}(Q)\right\rangle_{Q \subseteq N}$ in the state $s \in \mathrm{S}$. The matrix $p=(p(\sigma \mid s))_{\sigma, s \in \mathrm{S}}$ is a column stochastic matrix of transitional probabilities: for any $\sigma$ and $s$ in $\mathbf{S}, p(\sigma \mid s)$ is the transition probability from state $s$ to state $\sigma$. We assume that the players' preferences over utility streams can be represented by the expected discounted utility function. The discount factor is given by $\delta \in(0,1)$.

Let, $C$ be a non-empty convex subset of $\mathbb{R}^{n}$. Then the recession cone $\mathcal{O}^{+}[C]$ of $C$ is a set consisting of all vectors $y \in \mathbb{R}^{n}$ such that $C+y \subseteq C$. The lineality space of $C$ is a set of all vectors $y \in \mathbb{R}^{n}$ such that $C+y=C$.

We make the following assumption:

\section{Assumption (A).}

(Ail) $U_{s}$ is an NTU-game for all $s \in \mathbf{S}$.

(A2) The set $U_{s}(Q)$ is convex for all $s \in \mathrm{S}$ and $Q \subseteq N$.

(A3) For each $Q \subseteq N$ the set $U_{s \in \mathrm{S}} U_{s}(Q)$ is contained in a half-space of $\mathbb{R}^{n}$.

\footnotetext{
${ }^{1}$ For a comprehensive study of recession cones and related concepts see Rockafellar [29].
} 
(A4) For each $Q \subseteq N$, if $z_{1}, \ldots, z_{s}$ are vectors such that $z_{s} \in \mathcal{O}^{+}\left[U_{s}(Q)\right]$ and $\sum_{s \in s^{2}} z_{s}=0$, then $z_{s}$ belongs to the lineality space of $U_{s}(Q)$ for all $s \in \mathbf{S}$.

(A5) $\left(\sum_{s \in S} \mathcal{O}^{+}\left[U_{s}(N)\right]\right) \cap \mathbb{R}_{+}^{n}=\{0\}$

(A6) The matrix $p$ is irreducible.

Assumption (A1) requires the collection of sets $U_{s}$ to satisfy conditions (G1)-(G3) introduced in Chapter 2.

The convexity assumption (A2) is essential for most of our results. It ensures that a coalition that is able to improve upon a given utility stream can do so using a stationary utility stream. This property of the model makes it possible to restrict attention only to stationary utility streams and characterize a subgame of the stationary cooperative game by a static evaluation game. Without the convexity assumption, non emptiness of the classical core (let alone the non-emptiness of the strong sequential core) becones a considerably more complicated problem.

Assumptions (A3), (A4), and (A5) guarantee that the sum of the NTU games $U_{s}$ over $s \in S$ is an NTU-game. Assumption (A3) ensures that the sum of the sets $U_{s}(Q)$ is a proper subset of $\mathbb{R}^{r}$, while (A4) guarantees that it is a closed set. Together assumptions (A4) and (A5) gnarantee that the sum of the sets $U_{s}(N)$ intersected with a set of individually rational payoffs is bounded.

In particular, assumptions (A3)-(A5) are satisfied if the instantaneous NTU games $U_{s}$ satisfy condition (G4): there exists a real number $M$ such that for all $Q \subseteq N, s \in \mathbf{S}$, $u \in U_{s}(Q)$, and $i \in Q$ the inequality $u^{i} \leq M$ holds. In this case the recession cone of the set $U_{s}(Q)$ in given by

$$
\mathcal{O}^{+}\left[U_{s}(Q)\right]=\left\{z \in \mathbb{R}^{n} \mid z^{i} \leq 0 \text { for all } i \in Q\right\}
$$

and the limeality space of $U_{3}(Q)$ is the set

$$
\left\{z \in \mathbb{R}^{n} \mid z^{i}=0 \text { for all } i \in Q\right\}
$$

Of course, assumptions (A3)-(A5) are much more general. However, they restrict the ways in which the sets $U_{s}(Q)$ may be unbounded.

Assumption (A6) ensures that for all $s$ and $\sigma$ in $\mathrm{S}$ there is a non-zero probability of ever reaching state of from state $s$.

Assumption (A) is sufficient for most of the results reported in the chapter. For Theorem 7.1 and Corollary 7.1 , however, the following strengthening of (A) is needed:

Assumption (A*). Assumptions (A1)-(A5) are satisfied and, in addition, (A7) The set $U_{s}(N)$ is strictly convex for all $s \in \mathbf{S}$.

(A8) $p(\sigma \mid s)>0$ for all $s, \sigma \in \mathbf{S}$. 
A stationary cooperative game is a game with infinite time horizon, so the set of time periods or dates is $\mathrm{T}=\{0,1, \ldots t, \ldots\}$. At each date $t \in \mathrm{T}$ the state takes on one of the values from the set $\mathbf{S}$ determining the instantaneous $N T U$-game to be played at date $t$. The state follows a Markov process with one-step transitional probabilities given by $p$. The state at date $t=0$ is known to be $s_{0} \in \mathbf{S}$.

The set $D$ of all finite histories of the state of nature, the subset $\mathbb{D}(\xi)$ of $\mathbb{D}$ consisting of all finite histories that agree with $\xi$ up to the moment of time $t(\xi)$, and the probability $\rho(\xi \mid \eta)$ of the history $\xi$ conditional on $\eta$ are defined as in Chapter 6. A function $U$ from $\mathbb{D}$ into $\mathbb{R}^{n}$ will be referred to as a utility stream. We write $\mathcal{U}_{\xi}$ to denote the values for the function $\mathcal{U}$. The expected present value of the utility stream $\mathcal{U}$ conditional on the history $\xi \in \mathbb{D}$ is given by the function

$$
\mathcal{V}_{\mathbb{D}(\xi)}(\mathcal{U})=\sum_{\eta \in \mathbb{D}(\zeta)} \rho(\eta \mid \xi) \delta^{l(\eta)-l(\xi)} \mathcal{U}_{\eta}
$$

Given a stationary cooperative game $\Gamma$ and a finite history $\xi$ of the state of nature, let $\Gamma_{\mathbb{D}(\xi)}$ denote the subgame of $\Gamma$ starting at the node $\xi$.

Definition 7.1 The utility stream $\mathcal{U}$ belongs to the classical core $C\left(\Gamma_{\mathbb{E}(\xi)}\right)$ of the subgame $\Gamma_{\text {Du( }(\zeta)}$ if

1. $\mathcal{U}_{\eta} \in U_{s\left(\eta_{\eta}\right)}(N)$ for all $\eta \in \mathbb{D}(\xi)$, and $\mathcal{V}_{\mathbb{D}(\xi)}(\mathcal{U})$ is finite;

2. there exist no coalition $Q \subseteq N$ and no utility stream $\mathcal{Y}$ such that $\mathcal{Y}_{\eta} \in U_{s(\eta)}(Q)$ for all $\eta \in \mathbb{D}(\xi), \mathcal{V}_{\mathbb{D}(\xi)}(\mathcal{Y})$ is finite, and $\mathcal{V}_{\mathbb{D}(\xi)}^{i}(\mathcal{Y})>\mathcal{V}_{\mathbb{B}(\xi)}^{i}(\mathcal{U})$ for all $i \in Q$.

Definition 7.2 The strong sequential core of the stationary cooperative game $\Gamma$ is the set of utility streams

$$
S S C(\boldsymbol{\Gamma}):=\bigcap_{\xi \in \mathbb{D}} C\left(\boldsymbol{\Gamma}_{\mathbb{E}(6)}\right)
$$

\subsection{A characterization of the strong sequential core}

A utility strean $U$ is said to be stationary if its value $U_{\xi}$ at $\xi \in \mathbb{D}$ depends only on the current state $s(\xi)$ and does not depend on time or the history of the state of nature preceding the current date $t(\xi)$. For any stationary utility stream $\mathcal{U}$, there is an $(n S)$ dimensional vector $u=\left(u_{1}, \ldots, u_{S}\right)$ with $u_{s} \in \mathbb{R}^{n}$ such that $\mathcal{U}_{\xi}=u_{s(\xi)}$ for all $\xi \in \mathbb{D}$; we write $\mathcal{U}=\{u\}$.

Let $\psi=\left(I_{S}-\delta p\right)^{-1}$ be an $(S \times S)$-dimensional matrix. Let $\psi(\sigma \mid s)$ be the entry of $\psi$ at the intersection of row $\sigma$ and column s. By the same argument as in the proof of Proposition 6.1, one establishes the equation

$$
\mathcal{V}_{\mathbb{D}(\xi)}(\{u\})=\sum_{\sigma \in \mathrm{S}} \psi(\sigma \mid s(\xi)) u_{\sigma}
$$


Definition 7.3 The evaluation game $\mathscr{G}_{s}$ is a cooperative game where the possibilities of a coalition $Q$ are represented by the set $\times_{\sigma \in S} U_{\sigma}(Q)$, and the players' utilities from consuming the state-contingent utility tuple $u=\left(u_{1}, \ldots, u_{S}\right)$ are given by the $n$-vector

$$
v_{s}(u)=\sum_{\sigma \in s} \psi(\sigma \mid s) u_{\sigma} .
$$

The core $C\left(\mathscr{G}_{s}\right)$ of the evaluation game $\mathscr{G}_{s}$ consists of all state-contingent allocations $u$ in $x_{\sigma \in \mathrm{S}} U_{\sigma}(N)$ such that there is no $Q \subseteq N$ and no $y \in x_{\sigma \in \mathrm{S}} U_{\sigma}(Q)$ with $v_{s}^{i}(y)>v_{s}^{i}(u)$ for all $i \in Q$.

The role of the evaluation games in our analysis is similar to that of the evaluation economies defined in Chapter 6 . One can think of the feasibility set $x_{\sigma \in S} U_{\sigma}(Q)$ as consist. ing of all stationary utility streams feasible for the coalition $Q$. The utility function $v_{s}$ of the evaluation game $\mathscr{G}_{s}$ gives the expected present value of a stationary utility stream $\{u\}$, conditional on any finite history of the state of nature with the current state $s(\xi)=s$.

Closely related to the evaluation game $\mathscr{G}_{s}$ is the NTU game $V_{s}$ defined by the equation

$$
V_{s}(Q)=\sum_{\sigma \in \mathrm{S}} \psi(\sigma \mid s) U_{o}(Q)
$$

Thus the set $V_{s}(Q)$ consists of the expected present values of all $Q$-feasible stationary utility streams. Clearly, $u \in C\left(\mathscr{G}_{s}\right)$ if and only if $v_{s}(u) \in C\left(V_{s}\right)$.

Proposition 7.1. below states that the collection $V_{s}$ satisfies conditions (G1)-(G3). It is for Proposition 7.1 that the asstmptions (A4) and (A5) are crucial: they guarantee that $V_{s}(Q)$ is a closed set, and that the intersection of $V_{s}(N)$ with the set of individually rational payoffs is bounded.

Proposition 7.1 Let $\Gamma$ be a stationary cooperative game satisfying cussumption (A). Then $V_{s}$ is an $N T U$-game.

Proof. Let $Q$ be a non-empty subset of $N$. Then $V_{s}(Q)$ is obviously a non-empty set. It is entirely contained in a half-space of $\mathbb{R}^{n}$, by assumption (A3). To prove that $V_{s}(Q)$ is a closed set, observe that for any positive number $\lambda$ and any convex subset $C$ of $\mathbb{R}^{\prime \prime}$, the recession cone of the set $\lambda C$ coincides with that of the set $C$. In particular,

$$
\mathcal{O}^{+}\left[\psi(\sigma \mid s) U_{\sigma}(Q)\right]=\mathcal{O}^{+}\left[U_{\sigma}(Q)\right] .
$$

From this, from Assumption (A4) and Corollary 9.1 .1 in Rockafellar $\left[29\right.$, p. 74-75], $V_{s}(Q)$ is a closed set and its recession cone is given by

$$
\mathcal{O}^{+}\left[V_{s}(Q)\right]=\sum_{\sigma \in \mathrm{S}} \mathcal{O}^{+}\left[U_{\sigma}(Q)\right]
$$

Condition (G2) and the first part of condition (G3) can be easily werified. To prove that the set $V_{s}(N) \bigcap\left\{v \in \mathbb{R}^{n} \mid v \geq m\right\}$ is bounded we show its recession cone to contain only 
a zero vector. By Theorem 8.4 from $[29$, p. 64], any non-empty closed convex set whose recession cone consists only of the zero vector is bounded. By Corollary 8.3.3 from [29, p. 64], if the intersection of two closed convex sets is non-empty, then its recession cone equals the intersection of the respective recession cones. Thus, we compute:

$$
\begin{array}{r}
\mathcal{O}^{+}\left[V_{s}(N) \cap\left\{v \in \mathbb{R}^{n} \mid v \geq m\right\}\right]= \\
=\mathcal{O}^{+}\left[V_{s}(N) \| \cap \mathcal{O}^{+}\left[\left\{v \in \mathbb{R}^{n} \mid v \geq m\right\}=\right.\right. \\
=\left(\sum_{\sigma \in S} \mathcal{O}^{+}\left[U_{o r}(N)\right] \cap \bigcap \mathbb{R}_{+}^{*}=\{0\} .\right.
\end{array}
$$

The last of these equalities follows from assumption (A5).

Theorem 7.1. provides a criterion for a given stationary utility stream to be an element of the strong sequential core of a stationary cooperative game.

Theorem 7.1 Let $\Gamma$ be a stationary cooperative game satisfying Assumption (A). Let a stationary utility stream $\mathcal{U}=\{u\}$ be given. Then

l. $\mathcal{U} \in C\left(\boldsymbol{\Gamma}_{\mathrm{W}(\xi)}\right)$ if and only if $u \in C\left(\mathscr{G}_{\sin (\xi)}\right)$.

2. $U \in S S C(\Gamma)$ if and only if $u \in C\left(G_{s}\right)$ for all $s \in \mathbf{S}$.

Proof. To prove the first part of Theorem 7.1 we demonstrate that a coalition $Q \subseteq N$ is able to improwe upon $U$ in the subgame $\Gamma_{\mathbb{D}(s)}$ if and only if it can improve upon $u$ in the evaluation game $G_{s(\xi)}$. The second part of Theorem 7.1 is an immediate corollary of the frist part.

Suppose that there is a deviation $y$ from $u$ by a coalition $Q$ in the evaluation game

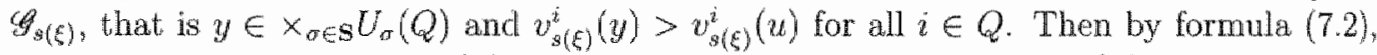
the stationary utility stream $\{y\}$ is a devation from the utility stream $\{u\}$ in the subgame $\Gamma(s)$.

Suppose that there is a devation from $\{u\}$ by a coalition $Q$ in the subgame $\Gamma_{g(g)}$, 1.e. the utility stream $\mathcal{Y}$ such that $\mathcal{Y}_{\eta} \in U_{s(\eta)}(Q)$ for all $\eta \in \mathbb{D}(\xi), V_{\mathbb{D}(\xi)}(\mathcal{Y})$ is finite, and $\mathcal{V}_{\mathrm{B}}\left(\xi_{)}(\mathcal{Y})>\mathcal{V}_{\mathrm{D}(\xi)}(\{u\})\right.$ for all $i \in Q$. Note that the utility stream $\mathcal{Y}$ need not be stationary. However, as we denonstrate in the remainder of the proof, coalition $Q$ can improve upon $\mathcal{U}$ using the stationary utility strean $\{z\}$. Convexity of the feasibility sets plays the central role in our axgument.

Choose the vectors $y_{s}$ arbitrarily in $U_{s}(Q)$ for all $s \in \mathrm{S}$. For every date $T \in \mathrm{T}$ define the process $\mathcal{Y}(T)$ by

$$
y_{n}(T)= \begin{cases}y_{\eta}, & \text { if } t(n)<T \\ y_{s(n)}, & \text { otherwise. }\end{cases}
$$

Observe that the process $\mathcal{Y}(T)$ is stationary as of date $T$ and therefore is bounded. Since the expected present value of $\mathcal{Y}(T)$ converges to that of $\mathcal{Y}$ as $T$ goes to infinity, there is a date $T_{0} \in \mathrm{T}$ such that

$$
\mathcal{V}_{\mathrm{B})}^{i}\left(\mathcal{Y}\left(T_{0}\right)\right)>\mathcal{V}_{\mathrm{D}(\mathrm{g})}(\{u\}) \text { for all } i \in Q
$$


Next, define the utility tuples $z_{\sigma}$ for all $\sigma \in \mathrm{S}$ by the equation

$$
z_{\sigma}=\frac{1}{\psi(\sigma \mid s(\xi))} \sum_{\substack{\eta \in \mathbb{N}(\xi) \\ s(\eta)=\sigma}} \rho(\eta \mid \xi) \delta^{\ell(\eta)-l(\xi)} \mathcal{Y}_{\eta}\left(T_{0}\right) .
$$

Because the process $\mathcal{Y}\left(T_{0}\right)$ is bounded, the sum in the above equation is well-defined. Therefore, $z_{\sigma}$ is a weighted average of the values for the process $\mathcal{Y}\left(T_{0}\right)$ over all nodes $\eta$ in the subtree $\mathbb{D}(\xi)$ with $s(\eta)=\sigma$. For all such nodes, $\mathcal{Y}_{\eta}\left(T_{0}\right) \in U_{\sigma}(Q)$. Since the set $U_{\sigma}(Q)$ is closed and convex, $z_{i \sigma} \in U_{\sigma}(Q)$. Using Equation (7.2), we compute:

$$
\begin{aligned}
\nu_{\mathbb{D}(\xi)}(\{z\}) & =\sum_{\sigma \in \mathrm{S}} \sum_{\substack{\eta \in \mathbb{D}(\xi) \\
(\eta \eta)=\sigma}} \rho(\eta \mid \xi) \delta^{t(\eta)-t(\xi)} \mathcal{Y}_{\eta}\left(T_{0}\right) \\
& =\sum_{\eta \in \mathbb{D}(\xi)} \rho(\eta \mid \xi) \delta^{t(\eta)-i(\xi)} \mathcal{Y}_{\eta}\left(T_{0}\right) \\
& =\mathcal{V}_{\mathbb{D}(\xi)}\left(\mathcal{Y}\left(T_{0}\right)\right) .
\end{aligned}
$$

We see that the stationary utility stream $\{z\}$ is deviation from $\{u\}$ by the coalition $Q$ in the sulbgame $\Gamma_{\mathbb{D}(\xi)}$. Therefore, $z$ is a deviation from $u$ by $Q$ in the evaluation game $\mathscr{G}_{\mathbb{a}(\hat{G})}$.

Theorem 7.2 gives sufficient conditions for the classical core of the stationary cooperative game to contain only stationary utility streams.

Theorem 7.2 Let $\Gamma$ be a stationary cooperative game satisfying Assumption ( $\mathbf{A}^{*}$ ). Then all utility streams in the classical core of $\Gamma$ are stationary.

Proof. Consider an $N$-feasible utility stream $\mathcal{U}$ with a finite expected present value at time $t=0$. If $U$ is not stationary, then there exist histories $\lambda, \varsigma \in \mathbb{D}$ such that $s(\lambda)=s(\varsigma)$ and $\mathcal{U}_{\lambda} \neq \mathcal{U}_{\varsigma}$. Note that both $\mathcal{U}_{\lambda}$ and $\mathcal{U}_{\varsigma}$ are the elements of the set $U_{s(\lambda)}(N)$. Under Assumption (A8), the probabilities $\rho(\lambda)$ and $\rho(\zeta)$ of $\lambda$ and $\varsigma$ conditional on the initial node $\left(0, s_{0}\right)$ are both positive. Strict convexity of the set $U_{s(\lambda)}(N)$ therefore implies that the vector

$$
u=\frac{\delta^{\prime(\lambda)} \rho(\lambda) \mathcal{U}_{\lambda}+\delta^{\prime(\varsigma)} \rho(\varsigma) \mathcal{U}_{\varsigma}}{\delta^{l(\lambda)} \rho(\lambda)+\delta^{\prime(\varsigma)} \rho(\varsigma)}
$$

belongs to the interior of $U_{s(\lambda)}(N)$. Therefore, there exists a vector $y \in U_{s(\lambda)}(N)$ such that $y^{i}>u^{i}$ for all $i \in N$. Define the utility stream $\mathcal{Y}$ as follows:

$$
\mathcal{Y}_{\eta}= \begin{cases}\mathcal{U}_{\eta}, & \text { if } \eta \neq \lambda, \varsigma \\ y, & \text { if } \eta=\lambda, \text { or } \eta=\varsigma .\end{cases}
$$

The utility stream $\mathcal{Y}$ is feasible for the caalition $N$, the expected present value of $\mathcal{Y}$ is finite, and is greater than that of the utility stream $\mathcal{U}$ at $t=0$, for each player in $N$. Hence, $\mathcal{Y}$ 
is an improvement upon $\mathcal{U}$ by the grand coalition at date $t=0$.

We conclude this section with a characterization of the strong sequential core.

Corollary 7.1 Let $\boldsymbol{\Gamma}$ be a stationary cooperative game satisfying Assumption ( $\mathrm{A}^{*}$ ). Then

$$
\operatorname{SSC}(\mathbf{\Gamma})=\left\{\{u\} \mid u \in \bigcap_{s \in \mathbb{S}} C\left(\mathscr{G}_{s}\right)\right\} .
$$

\subsection{Additive $\pi$-balancedness and strong additive $\pi$-balancedness}

In this section we consicer two balancedness conditions: so-called additive $\pi$-balancedness and strong additive $\pi$-balancedness. A special case of the former was used by Billera and Bixby in a series of papers on the characterization of market games (see, for example, [6] and [5]). It turns out that, when applied to the instantaneous NTU-game $U_{s}$ for all $s \in \mathrm{S}$, additive $\pi$-balancedness is sufficient for non-emptiness of the classical cores of all subgames of $\boldsymbol{\Gamma}$. Moreover, if at least one of the NTU-games $U_{s}$ is strongly additively $\pi$-balanced and the discount factor $\delta$ is close enough to one, then also the strong sequential core is non-emply.

In what follows $\mathcal{N}^{*}$ denotes the collection of non-empty proper subsets of the set $N$.

Definition 7.4 Let $V$ be a game with non-transferable utility and let $\pi$ be an element of the set $\Delta .^{2}$ The game $V$ is said to be additively $\pi$-balanced provided that the following condition is satisfied: If $\beta \subseteq \mathcal{N}^{*}$ is a $\pi$-balanced collection of coalitions, $\lambda \in \Delta_{\mathcal{N}}$ is a vector of balancing weights, $v_{Q} \in V(Q)$ for all $Q \in \beta$, then any vector $v_{N} \in \mathbb{R}^{n}$ satisfying the system of equations

$$
\pi_{N}^{i} v_{N}^{i}=\sum_{Q \in \beta^{\beta}} \lambda_{Q} \pi_{Q}^{i} v_{Q}^{i}, \forall i \in N
$$

belongs to the set $V(N)$. The NTU-game $V$ is said to be strongly additively $\pi$-balanced provided that any such vector $v_{N}$ belongs to the interior of $V(N)$.

We have thus defined the additive $\pi$-balancedness condition with respect to an arbitrary element $\pi$ of the set $\Delta$. However, if the game $V$ is additively $\pi$-balanced, then $\pi_{N}^{i}>0$ for all $i \in N$. Indeed, suppose that $N_{+}=\left\{i \in N \mid \pi_{N}^{i}>0\right\}$ is a proper subset of $N$. Observe that the collection of singletons $\beta=\left\{\{i\} \mid i \in N_{+}\right\}$is a $\pi$ balanced collection of coalitions. Let $m^{i}$ be the individually rational payoff of the player is, i.e. a real number such that $V(\{i\})=\left\{x \in \mathbb{R}^{n} \mid x^{i} \leq m^{i}\right\}$. Then the additive $\pi$-balancedness condition implies that $V(N)$ contains the unbounded set $\left\{v \in \mathbb{R}^{n i} \mid v^{i}=m^{i}\right.$ for all $\left.i \in N_{+}\right\}$, thus violating the condition (G3).

The property of (strong) additive $\pi$-balancedness is additive over games in the following sense.

\footnotetext{
${ }^{2}$ See Chapter 2 for the definitions of $\Delta$ and of $\%$ balanced collection of coalitions.
} 
Proposition 7.2 Let $\mathbf{\Gamma}$ be a stationary cooperative game satisfying Assumption (A), and let $\pi \in \Delta$. Suppose that the instantaneous $N T U$-games $U_{s}$ are additively $\pi$-balanced in all states $s \in \mathrm{S}$. Then the NTU-games $V_{s}$ are additively $\pi$-balanced for all $s \in \mathrm{S}$. If , mareover, at least one of the instantaneous NTU-games is strongly additively it-balanced, then the NTU-games $V_{s}$ are strongly additively $\pi$-balanced for all $s \in \mathrm{S}$.

Proof. Let a $\pi$-balanced collection of coalitions $\beta \subseteq \mathcal{N}^{*}$, a vector of balancing weights $\lambda \in \Delta_{N}$, and the vectors $v_{Q} \in V_{s}(Q)$ for all $Q \in \beta$ be given. We already know that $\pi \pi_{N}^{i}>0$ for all $i \in N$, implying that the system of equations (7.4) defines a unique vector $v_{N}$.

Choose the vectors $u_{Q \sigma} \in U_{\sigma}(Q)$ so that

$$
v_{Q}=\sum_{\sigma \in \mathrm{S}} \psi(\sigma \mid s) u_{Q \sigma}
$$

for all $Q \in \beta$, and let the vectors $u_{N_{\sigma}}$ be defined by the system of equations

$$
\pi_{N}^{i} u_{N \sigma}^{i}=\sum_{Q \in \beta} \lambda_{Q} \pi_{Q}^{i} u_{Q \sigma}^{i}, \forall i \in N
$$

Then

$$
v_{N}=\sum_{\sigma \in \mathrm{S}} \psi(\sigma \mid s) u_{N \sigma}
$$

If the instantaneous games $U_{\sigma}$ are additively $\pi$-balanced in all states, then the vector $u_{N}$ belongs to the set $U_{\sigma}(N)$ for $\sigma \in \mathrm{S}$, implying that $v_{N}$ is an element of the set $V_{*}(N)$. If, in addition, at least one of the instantaneous NTU-games, say the game $U_{\partial}$, is strongly additively $\pi$-balanced, then $u_{N \bar{\sigma}}$ is in the interior of the set $U_{\bar{\sigma}}(N)$. Since the number $\psi(\bar{\sigma} \mid s)$ is positive, the vector $v_{N}$ belongs to the interior of the set $V_{s}(N)$.

It is straightforward to show that any additively $\pi$-balanced NTU game $V$ is $\pi$ balanced in the sense of Billera [4] (see Chapter 2). It follows that an additively $\pi$-balanced game has a non-empty core. Define the proper core of the NTU game $V$ as the set of utility payolts

$$
P(V)=\partial V(N) \backslash \bigcup_{Q \in N^{*}} V(Q) .
$$

Proposition 7.3 If the $N T U$-game $V$ is strongly additively $\pi$-balanced, then the proper core of $V$ is non-empty.

Proof. Let $V$ be a strongly additively $\pi$-balanced NTU-game. For any $\pi$-balanced collection of coalitions $\beta \subseteq \mathcal{N}^{*}$ let the set $W(\beta)$ be defined by

$$
W(\beta)=\bigcap_{Q \in \beta} V(Q)
$$


Define the NTU-game $V^{*}$ as follows. Let $V^{*}(Q)=V(Q)$ for all proper subsets $Q$ of $N$, and let $V^{*}(N)$ be the union of $W(\beta)$ over all $\pi$-balanced collections $\beta \subseteq N^{*}$. We leave the verification of conditions $(\mathrm{G1})-(\mathrm{G} 3)$ to the reader. By construction, the NTU-game $V^{*}$ is $\pi$-balanced, and therefore has a non-empty core. Let $v^{*}$ be a core element of the game $V^{*}$.

Since $V$ is strongly additively $\pi$-balanced, the inclusion $W(\beta) \subset$ int $V(N)$ holds for any $\pi$ balanced collection $\beta$. It follows that $V^{*}(N) \subset \operatorname{int} V(N)$. Consider the set

$$
T=\left\{t \in \mathbb{R} \mid\left(v^{*}+t 1_{N}\right) \in V(N)\right\},
$$

where $1_{N}$ is a vector in $\mathbb{R}^{n}$ with all components equal to one. As $v^{*}$ is an element of $V^{*}(N)$, it is also an element of $V(N)$. Hence, $0 \in T$. Since $V(N)$ is closed, $T$ is also closed. Moreover, $T$ is bouncied from above for otherwise the set $V(N)$ would coincide with $\mathbb{R}^{n}$, violating condition (G1). It follows that the the supremum $\bar{t}$ of the set $T$ is finite, and is an element of $T$.

We show that the vector $\bar{v}=v^{*}+\bar{t} 1_{N}$ belongs to the proper core of the game $V$. Since $\bar{t} \in T$, we have the inclusion $\bar{v} \in V(N)$. If $\bar{v}$ were an interior point of $V(N)$, then there would exist a $t>0$ such that $\left(\bar{v}+t \mathbf{1}_{N}\right)$ is in $V(N)$. Hence, $(\bar{t}+t)$ would be an element of $T$, a contradiction.

Since $0 \in T$, we know that $\bar{t} \geq 0$. If $\bar{t}$ were equal to zero, then $\bar{v}=v^{*}$ would be an element of $V^{*}(N)$ and therefore an interior point of $V(N)$. However, we already know that $\bar{v}$ is not in the interior of $V(N)$. So $\bar{t}>0$, and $\bar{y}^{i}>v^{* i}$ for all $i \in N$.

Finally, if $\bar{v}$ were an element of $V(Q)$ for some $Q \in \mathcal{N}^{*}$, then $v^{*}$ would be an interior point of $V(Q)$, contradicting the choice of $v^{*}$ in the core of the game $V^{*}$.

\subsection{Existence results}

We are now in a position to state our first existence result. If the instantaneous NTUgames are additively $\pi$-balanced in all states, then by Proposition 7.2 the evaluation games $y_{3}$ possess non-empty cores. Consequently every subgame of the stationary cooperative game has a non-empty classical core. Thus we have proved the following theorem.

Theorem 7.3 Let $\Gamma$ be a stationary cooperative game satisfying Assumption (A), and let $\pi \in \Delta$. Suppose that each of the instantaneous $N T U$-games $U_{s}, s \in \mathrm{S}$, is additively $\pi-b a l a n c e d$. Then the classical core of any subgame of $\Gamma$ is non-empty.

Let us now turn to the question of non-emptiness of the strong sequential core. We consider a family of stationary cooperative games $\Gamma_{\delta}$ parameterized by the discount factor $\delta \in(0,1)$. Let $\phi=(\phi(1), \ldots, \phi(S))$ be the stationary distribution over the set $\mathbf{S}$.

Definition 7.5 The game $\mathscr{G}_{\infty}$ is a cooperative game where the passibilities of a coalition $Q$ are represented by the set $x_{\sigma \in S} U_{\sigma}(Q)$, and the players" utilities from consuming the 
utility tuple $u \in \mathbb{R}^{n S}$ are given by the vector

$$
v_{\infty}(u)=\sum_{\sigma \in \mathrm{S}} \phi(\sigma) u_{\sigma}
$$

We say that the state-contingent utility tuple $u$ in $x_{\sigma \in s} U_{o}(N)$ belongs to the proper core of the game $G_{\infty}$ if there is no $y \in x_{\sigma \in S} U_{o r}(N)$ with $v_{s}^{i}(y)>v_{s}^{i}(u)$ for $a l l \in N$, and no $Q \in \mathcal{N}^{*}$ and $z \in x_{a \in s} U_{o}(Q)$ with $v_{s}^{i}(z) \geq v_{s}^{i}(u)$ for all $i \in Q$.

Define the NTU-game $V_{\infty}$ as follows:

$$
V_{\infty}(Q)=\sum_{\sigma \in S} \phi(\sigma) U_{\sigma}(Q)
$$

By the argument analogous to the proof of Proposition $7,1, V_{\infty}$ is satisfies conditions (G1)(G3), provided that the stationary cooperative game $\Gamma$ satisfies assumption (A). Clearly, $u$ is in the proper core of $\mathscr{G}_{\infty}$ if and only if $v_{\infty}(u)$ belongs to the proper core of the NTU-game $V_{\infty}$.

By Theorem 6.3 , the utility function $v_{6 s}$ in the evaluation game $\mathscr{G}_{s}$ converges to the function $v_{\infty}$ as $\delta$ approaches one, in the following sense:

$$
\lim _{\substack{\delta \rightarrow 1 \\ 0<\delta<1}}(1-\delta) v_{\delta s}(u)=v_{\infty}(u)
$$

Theorem 7.4 Let $\Gamma_{\delta}$ be a family of stationary cooperative games satisfying Assumption (A). Let $u$ be in the proper core of the game $G_{\infty}$. Then there exists a $\vec{\delta} \in(0,1)$ such that the stationary utility stream $\{\bar{u}\}$ is an elenent of the strong sequential core of the game $\mathbb{\Gamma}_{\delta}$ whenever $\bar{\delta}<\delta<1$.

Proof. We must show that, $\bar{u}$ belongs to the core of the evaluation game $\mathscr{G}_{s}$ for all $s \in \mathbf{S}$ and all d's sufficiently close to one. The argument consists of two steps.

Step 1. We show that $v_{s s}(u) \in \partial V_{s s}(N)$ for all $s \in \mathbf{S}$ and for all $\delta \in(0,1)$.

Since $v_{\infty}(u) \in \partial V_{\infty}(N)$, there exists a non zero vector $\alpha \in \mathbb{R}_{+}$such that $v_{\infty}(\bar{u})$ maximizes the scalar product $\alpha \cdot v$ over all $v \in V_{\infty}(N)$. Since $\phi(\sigma)$ is positive for all $\sigma \in \mathbf{S}$, $\bar{u}_{\sigma}$ maximizes $\alpha \cdot u_{\sigma}$ over all $u_{\sigma} \in U_{\sigma}(N)$. Therefore, $v_{s, s}(\bar{u})$ is a maximizer of $\alpha \cdot v$ over all $v \in V_{\delta B}(N)$, for all $s \in \mathrm{S}$ and $\delta \in(0,1)$. This implies that $w_{s,}(\tilde{u})$ is a boundary point of $V_{\delta ;}(N)$.

Step 2. We show that $v_{\tilde{s}}(\bar{u})$ is in the complement of $V_{s,}(Q)$ for all $s \in \mathrm{S}_{3} Q \in \mathcal{N}^{*}$, and all $\delta$ close to one.

Suppose not. Then there exists state $s \in \mathbf{S}$, coalition $Q \in \mathcal{N}^{*}$ and sequence $\delta^{\prime} \in(0,1)$ converging to one such that $v_{\delta}(\bar{u})$ is an element of $V_{b q_{s}}(Q)$ for all $q$. Let the subset $C$ of $\mathbb{R}^{n} \times \mathbb{R}^{S}$ be defined as

$$
C=U\left\{\sum_{\sigma \in \mathrm{s}} \gamma_{\sigma} U_{\sigma}(Q)\right\} \times\left\{\gamma_{1}, \ldots, \gamma_{s}\right\}
$$


where the mion is taken over all choices of non-negative coefficients $\gamma_{1}, \ldots, \gamma_{s}$ adding up to 1. By Equation (7.5) and Theorern 6.3, the sequence

$$
\left(1-\delta^{g}\right)\left[v_{\delta^{*}}(\bar{u}), \psi_{g^{4}}(1 \mid s), \ldots, \psi_{\delta^{q}}(S \mid s)\right]
$$

converges to the vector $\left[v_{\infty}(\bar{u}), \phi\right]$. Since each vector in the sequence is an element of the set $C$, the vector $\left[u_{\infty}(\bar{u}), \phi\right]$ belongs to the closure of $C$.

Let $\left\{e_{1}, \ldots, e_{S}\right\}$ be the standard ordered basis of $\mathbb{R}^{S}$, and let the subset $C_{\sigma}$ of $\mathbb{R}^{n} \times \mathbb{R}^{S}$ be defined as $C_{\sigma}=U_{\sigma}(Q) \times\left\{0_{\sigma}\right\}$. Observe that the set $C$ can be written as a union

$$
C=\bigcup\left\{\sum_{\sigma \in \mathrm{S}} \gamma_{\sigma} C_{\sigma}\right\} .
$$

It follows from Theorem 3.3 in Rockafellar [29, p. 18] that the set $C$ is the convex hull of the union $\bigcup_{\sigma \in \mathrm{S}} C_{\sigma}$. By Theorem 9.8 in Rockafellar [29, p. 80] and by Assumption (A4), the closure of $C$ is therefore given by

$$
\begin{aligned}
\mathrm{d} C & =\bigcup\left\{\sum_{\substack{\sigma \in \mathrm{S} \\
\gamma_{\sigma}>0}} \gamma_{\sigma} C_{\sigma}+\sum_{\substack{\sigma \in \mathrm{S} \\
\gamma_{\sigma}=0}} \mathcal{O}^{+}\left[C_{\sigma}\right]\right\} \\
& =\bigcup\left\{\sum_{\substack{\sigma \in \mathrm{S} \\
\gamma_{\sigma}>0}} \gamma_{\sigma} U_{\sigma}(Q)+\sum_{\substack{\sigma \in \mathrm{S} \\
\gamma_{\sigma}=0}} \mathcal{O}^{+}\left[U_{\sigma}(Q)\right]\right\} \times\left\{\gamma_{1}, \ldots, \gamma_{S}\right\} .
\end{aligned}
$$

Thus, the inclusion $\left[v_{\infty}(\bar{u}), \phi\right] \in \mathrm{cl} C$ together with the positivity of the probabilities $\phi(\sigma)$ implies that $v_{\infty}(\bar{u})$ is an element of $\sum_{a \in \mathrm{S}} \phi(\sigma) U_{\sigma}(Q)=V_{\infty}(Q)$. However, this contradicts the choice of $\bar{u}$ in the proper core of the game $\mathscr{G}_{\infty}$.

By an argument similar to the proof of Proposition 7.2 one can show that the NTUgame $V_{\infty}$ is strongly additively $\pi$-balanced, provided that the instantaneous NTU-games are additively $\pi$-balanced in all states, and at least one of these NTU-games is strongly additively $\pi$-balanced. In this case the game $\mathscr{G}_{\infty}$ has a non-empty proper core. We have thus established the following result.

Corollary 7.2 Let $\Gamma_{\delta}$ be a fanily of stationary cooperative games satisfying Assumption (A), and let $\pi \in \Delta$. Suppose that each of the NTU-games $U_{s}, s \in \mathbf{S}$, is additively $\pi$ balanced, and at lecst one of these NTU-games is strongly additively $\pi$-balanced. Then there exists $a \bar{\delta} \in(0,1)$ such that the strong sequential core of the game $\boldsymbol{\Gamma}_{\delta}$ is non-empty whenever $<<<1$.

Corollary 7.2 does not imply our earlier result asserting the non-emptiness of the strong sequential core in a stationary exchange economy with a sufficiently high discount factor. Indeed, the instantaneous games $U_{s}$ constructed using the data of the stationary exchange 
economy are additively $\pi$-balanced with respect to an element of the set $\Delta$ defined by the equatuions

$$
\pi_{Q}^{i}=\frac{1}{|Q|} \forall i \in Q
$$

where $|Q|$ is the cardinality of $Q$. However, these games may violate the strong additive $\pi$-balancedness condition for any $\pi$, even when the economy satisfes all assumptions of Corollary 6.2

\subsection{An example}

We construct an example of a family of stationary cooperative ganes where the strong sequential core is empty for all $\delta \in(0,1)$. In this example the stationary cooperative games satisfy assumption $\left(\mathrm{A}^{*}\right)$, and the NTU-game $U_{s}$ is additively $\pi$-balanced for all $s \in \mathrm{S}$. However, no $U_{s}$ has the property of strong additive $\pi$-balancedness.

Example 7.1 Consider a family $\mathbb{\Gamma}_{\delta}$ of stationary cooperative games with three players called $a, b$, and $c$, and two states of nature. The transitional probabilities are given by the matrix

$$
p=\left[\begin{array}{ll}
0.75 & 0.25 \\
0.25 & 0.75
\end{array}\right]
$$

The stationary distribution over $\mathbf{S}$ is given by the vector $\phi=(0.5,0.5)$. Table 7.1 reports the equations defining the sets of instantaneous utility payoffs.

Table 7.1: Equations defining the sets $U_{\sigma}(Q)$

\begin{tabular}{lllll}
\hline$Q$ & & $s=1$ & $s=2$ \\
$\{a\}$ & $\exp \left(u^{a}\right)$ & $\leq$ & 1 & 1 \\
$\{b\}$ & $\exp \left(u^{b}\right)$ & $\leq$ & 1 & 1 \\
$\{c\}$ & $u^{c}$ & $\leq$ & 1 & 1 \\
$\{a, b\}$ & $\exp \left(u^{a}\right)+\exp \left(u^{b}\right)$ & $\leq$ & 6 & 6 \\
$\{a, c\}$ & $\exp \left(u^{a}\right)+u^{c}$ & $\leq$ & 6 \\
$\{b, c\}$ & $\exp \left(u^{b}\right)+u^{c}$ & $\leq$ & 6 & 8 \\
$\{a, b, c\}$ & $\exp \left(u^{a}\right)+\exp \left(u^{b}\right)+u^{c}$ & $\leq 10$ & 10 \\
\hline
\end{tabular}

To prove that the assumptions (A4) and (A5) are satisfied, we show that for cacti coalition $Q$ and each state s the recession cone of the set $U_{s}(Q)$ is given by the set $\mathbb{R}^{Q}=$ $\left\{y \in \mathbb{R}^{n} \mid y^{i} \leq 0 \vee i \in Q\right\}$. The inclusion $\mathbb{R}_{-}^{Q} \subseteq \mathcal{O}^{+}\left[\mathcal{V}_{s}(Q)\right]$ follows automatically from condition (G2). For coalition $Q=\{a, b\}$ the converse inclusion follows from the fact that $u^{a} \leq \ln 6$ and $u^{*} \leq \ln 6$ for all $u \in U_{s}(\{a, b\})$. Consider the case where $Q=\{a, c\}$. Let the vector $y$ be an element of $\mathcal{O}^{+}\left[U_{s}(\{a, c\})\right]$. By definition of the recession cone, 
$y+u \in U_{s}(\{a, c\})$ whenever $u \in U_{s}(\{a, c\})$. This implies that the vector $y$ must satisfy the inequality

$$
\exp \left(u^{a}\right)\left(\exp \left(y^{a}\right)-1\right)+y^{c} \leq 0
$$

for all real numbers $u^{a}$. For $u^{a}$ approaching $-\infty$, this implies that $y^{c} \leq 0$. For $u^{a}$ approaching $+\infty$, it follows that $y^{a} \leq 0$. Thus, $y \in \mathbb{R}_{-}^{\{a, c\}}$, as clesired. The case of coalitions $\{b, c\}$ and $\{a, b, c\}$ is dealt with similarly.

Using the convexity property of the exponential function it is not difficult to show that the games $U_{3}$ are additively $\pi$-balanced with respect to $\pi$ defined by Equation (7.6). To see that the game $U_{1}$ violates the condition of strong additive $\pi$-balancedness for any $\pi \in \Delta$, observe that the core of $U_{1}$ consists of the vector $(\ln 4, \ln 2,4)$ alone, and that this vector is feasible for every coalition of size 2. Thus the proper core of $U_{1}$ is empty. A similar argument shows that the game $U_{2}$ violates the condition of strong additive $\pi$-balancedness.

Table 7.2 reports the equations defining the sets $V_{\infty}(Q)$ and the sets $V_{\delta s}(Q)$ deflated, for the simplicity of notation, by the factor of $(1-\delta)$. We use the notation

$$
\begin{aligned}
& w_{\delta s}(\{a, c\})=(1-\delta)\left[\psi_{\delta}(1 \mid s) \times 8+\psi_{\delta}(2 \mid s) \times 6\right] \\
& w_{\delta_{s}}(\{b, c\})=(1-\delta)\left[\psi_{\delta}(\mathbb{1} \mid s) \times 6+\psi_{\delta}(2 \mid s) \times 8\right]
\end{aligned}
$$

Table 7.2: Equations defining the sets $V_{\infty}(Q)$ and $(1-\delta) V_{\delta s}(Q)$

\begin{tabular}{lllcc}
\hline$Q$ & & & $V_{\delta s}(Q)$ & $V_{\infty}(Q)$ \\
\hline$\{a\}$ & $\exp \left(v^{a}\right)$ & $\leq$ & 1 & 1 \\
$\{b\}$ & $\exp \left(v^{b}\right)$ & $\leq$ & 1 & 1 \\
$\{c\}$ & $v^{c}$ & $\leq$ & 1 & 1 \\
$\{a, b\}$ & $\exp \left(v^{a}\right)+\exp \left(v^{b}\right)$ & $\leq$ & 6 & 6 \\
$\{a, c\}$ & $\exp \left(v^{a}\right)+v^{c}$ & $\leq$ & $w_{\delta s}(\{a, c\})$ & 7 \\
$\{b, c\}$ & $\exp \left(v^{b}\right)+v^{c}$ & $\leq$ & $w_{\delta s}(\{b, c\})$ & 7 \\
$\{a, b, c\}$ & $\exp \left(v^{a}\right)+\exp \left(v^{b}\right)+v^{c}$ & $\leq$ & 10 & 10 \\
\hline
\end{tabular}

Table 7.2 makes it clear that the core of the game $V_{\infty}$ is a one-point set

$$
C\left(V_{\infty}\right)=\left\{\left(\begin{array}{l}
v^{a} \\
v^{k} \\
v^{a}
\end{array}\right)=\left(\begin{array}{c}
\ln 3 \\
\ln 3 \\
4
\end{array}\right)\right\} .
$$

It follows that the core of the game $\mathscr{G}_{\infty}$ is a one-point set.

$$
C\left(\mathscr{G}_{\infty}\right)=\left\{\begin{array}{ll}
\left(\begin{array}{ll}
u_{1}^{a} & u_{2}^{a} \\
u_{1}^{b} & u_{2}^{b} \\
u_{1}^{c} & u_{2}^{c}
\end{array}\right) & \begin{array}{l}
u_{1}^{a}=u_{2}^{a}=\ln 3 \\
u_{1}^{b}=u_{2}^{b}=\ln 3 \\
u_{1}^{c}=u_{2}^{c}=4
\end{array}
\end{array}\right\}
$$


The core of the NTU-game $V_{s}$ and the core of the ewaluation game $G_{s}$ are given by

$$
\begin{gathered}
C\left(V_{\delta s}\right)=\left\{\left(\begin{array}{l}
v^{a} \\
v^{b} \\
v^{c}
\end{array}\right)=\left(\begin{array}{c}
\ln \left[w_{\delta_{s}}(\{a, c\})-4\right] \\
\ln \left[w_{\delta s}(\{b, c\}-4]\right. \\
4
\end{array}\right)\right\} \\
C\left(\mathscr{G}_{\delta s}\right)=\left\{\left(\begin{array}{ll}
u_{1}^{a} & u_{2}^{a} \\
u_{1}^{b} & u_{2}^{b} \\
u_{1}^{c} & u_{2}^{c}
\end{array}\right), \begin{array}{l}
u_{1}^{a}=u_{2}^{a}=\ln \left[w_{s s}(\{a, c\})-4\right] \\
u_{1}^{b}=u_{2}^{b}=\ln \left[w_{s}(\{b, c\})-4\right] \\
u_{1}^{c}=u_{2}^{c}=4
\end{array}\right\}
\end{gathered}
$$

Now one can check that $w_{\delta 1}(\{a, c\}) \neq w_{\delta 2}(\{a, c\})$ implying that the intersection $C\left(g_{\delta 1}\right) \cap$ $C\left(G_{\delta_{2}}\right)$ is an empty set for all $\delta \in(0,1)$. Corollary 7.1 implies that the strong sequential core of the game $\Gamma_{\delta}$ is empty for all $\delta \in(0,1)$. 



\section{Bibliography}

[1] J.-P. Aubin. Mathematical Methods of Game and Economic Theory. North Holland, Amsterdam, 1979.

[2] R.J. Aumann. The Core of a Cooperative Game without Side Payments. Transactions of the American Mathematical Society, 98: 539-552, 1961.

[3] R.J. Aumann and B. Peleg. Von Neumann-Morgenstern Solutions to Cooperative Games without Side Payments. Bulletin of the American Mathematical Society. 66 : $173-179,1960$.

[4] L.J. Billera. Some Theorems on the Core of an n-person Game without SidePayments. SIAM Journal of Applied Mathematics, 18: 567-579, 1970.

[5] L.J. Biller A. On Games Without Side Payments Arising from a General Class of Markets. Journal of Mothematical Economics, 1: 129-139, 1974.

6] L.J. Bhlera And R.E. Bixby. A Characterization of Polyhedral Market Games. International Joumal of Game Theory, 2: 253-261, 1973.

[7] O.N. Bondareva. Some Applications of Linear Programming Methods to the Theory of Cooperative Games. Problemy Kibernetiki, 10: 119-139, 1963.

[8] J.-M. Bonnisseau And V. IEhlé. Payofts-dependent Balancedness and Cores. Cahiers de la MSE Série Bleue ł2003.45, Université Paris-1, 2003.

[9] G. Debrev. Smooth Preferences. Econometrica, 40:603-615, 1972.

[10] M. Florenzano. General Equilibrium Analysis: Existence and Optimality Properties of Equilibria. Kluwer Academic Publishers, Boston, 2003.

[11] D. GALE. The Core of a Monetary Economy without Trust. Jowral of Economic. Theory, 19: 456 491, 1978 .

[12] P.J.J. Herings. An Extremely Simple Proof of the K-K-M-S Theorem. Economic Theory, 10: 361-367, 1997 . 
[13] P.J.J. Herings, G. VAn der LAAN, AND A.J.J. TALMAN. Socially Structured Games and Their Applications. METEOR Research Memorandum 03/09. University of Maastricht, 2003.

[14] F. Hüssenov. Interpretation of Aubin's Fuzzy Coalitions and Their Extension. Journal of Mathematical Economics, 23: 499-516, 1994.

[15] Y. KannaI. The Core and Balancedness. In R.J. Aumanm and S. Hart, editors, Handbook of Game Theory with Economic Applications, Chapter 12, 355-395. North-Holland, Amsterdam, 1992.

[16] H. Keiding AND L. Thorlund-PETERsen. The core of a cooperative game without side payments. Journal of Optimization Theory and Applications, 54: 273-288, 1987.

[17] L.C. Koutsougeras. A Two-Stage Core With Applications to Asset Market and Differential Information Economies. Economic Theory, 11: 563-584, 1998.

[18] L.C. Koutsougeras. On an Edgeworth Characterization of Rational Expectations Equilibria in Atomless Asset Market Economies. July 2002.

[19] L.C. Koutsougeras and W.J. Shafer. The Rational Expectations Core. Work in progress.

[20] L. Kranich, A. Perea, and H. Peters. Core Concepts for Dynamic TU-game. METEOR Research Memorandum 01/024, Universiteit Maastricht, 2001. Forthcoming in International Game Theory Review.

[2]] V. MARAKulin. Contracts and Domination in Incomplete Markets. EERC working paper 02/04.

[22] A. Mas-Coleli, M.D. Whinston, And J.R. Green. Mictoeconomic Theory. Oxford University Press, New York, 1995.

[23] A. Predtetchinsk. The Fuzzy Core and the (II, $\beta$ ) Balanced Core. METEOR Research Memorandum 04/025, University of Maastricht, 2004. Forthcoming in Economic Theory.

[24] A. Predtemchinski and P.J.J. Herings. A Necessary and Sufficient Condition for Non-emptiness of the Core of a Non-transferable Utility Game. Journal of Economic Theory, 116: 84-92, 2004.

[25] A. Predtetchinsri, P.J.J. Herings, and A. Perea. The Weak Sequential Core for Two-period Economies. METEOR Research Memorandum 02/011, University of Maastricht, 2002.

[26] A. Predtetchinski, P.J.J. Herings, and H. Peters. The Strong Sequential Core in a Dynamic Exchange Economy. Economic Theory, 24: 147-162, 2004. 
[27] A. Predtetchinski, P.J.J. Herings, and H. Peters. The Strong Sequential Core for Two-period Economies. Joumal of Mathematical Economics, 38: 465-482, 2002.

[28] D. RAY. Credible Coalitions and the Core. International Journal of Came Theory, 18: 185-197, 1989 .

[29] R.T. Rockafellar. Convex Analysis. Princeton University Press, Princeton, New Jersey, 1997.

[30] H. SCARF. The Core of an n-person Game. Econometrica $a_{n} 35: 50-69,1967$.

[31] L.S. ShapleY. On Balanced Sets and Cores. Navel Research Logistics Quartely, 14: $453-460,1967$.

[32] L.S. Shapley. On Balanced Games without Side Payments. In T.C. Hu and S.M. Robinson, editors, Mathematical Programming, 261-290. Academic Press, New-York, 1973.

[33] G. VAn der LaAn, A.J.J. TAlman, And Z. Yang. Cooperative Games in Permutational Structure. Economic Theory, 11: 427-442, 1998.

[34] J. von Neumann And O. Morgenstern. Theory of Games and Economic Behavior. Princeton University Press, Princeton, N.J., 2nd ed., 1947. 


\section{Index}

allocation

constrained $M$-feasible, 48

constrained feasible, 33

stationary, 71

assets, 33

strongly complete set of, 40

balancedness

$(\pi,<)$-balancedness, 12

IT-balancedness, 12

$\Psi$-balancedness, 17

$\pi$-balancedness, 11

additive $\pi$-balancedness, 90

strong additive $\pi$-balancedness, 90

weak $(\pi,<)$-balancecness, 12

Billera, 11

evaluation, 72

finance, 37

stationary exchange, 68

two-period, 33

game

evaluation, 87

stationary cooperative, 84

with non-transferable utility, 11

Iehlé, 16

K-K-M-S Theorem, 17

Keiding, 11

Koutsougeras, 50

Thorlund-Petersen, 11

Bonnisseau, 16

core

$\Psi$-balanced, 17

e-fizzy, 23

classical

of the ex ante subeconomy, 34

of the ex post subeconomy, 34,48

of the subeconomy $\mathbf{E}_{\mathbb{D}(k)}, 70$

of the subgame $\Gamma(\xi), 86$

fuzzy, 22

of the game with non-transferable util-

ity, 11

rational expectations, 5

strong sequential, $36,70,86$

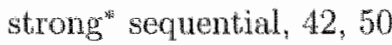

two-stage, 50

weak sequential, 49

economy 


\section{Summary}

This thesis presents a study of the cooperative solution concept known as the core in several game-theoretic and economic contexts. Two principal topics covered in the thesis are: (I) Payoff-dependent balancedness and the core, and (II) Sequential core concepts. The thesis is correspondingly divided into two parts. Two parts of the thesis differ in the subject matter as well as in the methodology of research. Part. I. should be seen as a relatively technical part of the thesis. Part II is intended as an introduction to the dynamic cooperative game theory, a new discipline that receives increasing attention in the literature. The challenges in the second part have a conceptual rather than technical nature.

Chapter 2 of the thesis elaborates on the classical problem of the non-emptiness of the core in games with non-transferable utility. In the class of games with non-transferable vitility sufficient conditions for the non-emptiness of the core, known as balancedness conditions, were given in Scarf [30] and in Billera [4]. These conditions are known to be excessively restrictive, in the sense that there exist games having a non-empty core that do not satisfy Scarf's, or even a weaker Billera's balancedness conditions.

Chapter 2 closes a gap between necessary and sufficient conditions for the non-emptiness of the core. We introduce a new condition for the non-emptiness of the core, the so-called $\Pi$-balancedness condition, as a natural generalization of the balancedness condition of Billera [4]. The main result of Chapter 2 states that $\Pi 1$-balancedness is a necessary and sufficient condition for the non-emptiness of the core in a general non transferable utility game. Chapter 2 also contains a discussion of the concept of $\Psi$-balenced core introduced recently in Bonnisseau and lehié [8].

Chapter 3 presents an application of the idea of payolf-dependent balancedness. Wo give a new proof of the non-emptiness of the fuzzy core in a pure exchange cconomy with finitely many agents. The proof reveals and exploits an interesting connection between the fuzzy core of a pure exchange economy and the $\Psi$-balanced core of a game with nor transferable utility.

The traditional cooperative game theory considers the core along with other solution concepts in static and deterministic contexts such as games with non-transferable utility and one-period economies. The cooperation that takes place in dynamic and uncertain environments is a subject of a new line of research that attracts increasing attention of the scholars. Thus, a number of core concepts has recently been proposed in the literature for specific game-theoretic and economic situations where time and uncertainty play an 
essential ralle. Part II of this thesis seeks to extend the concept of the core to the class of economies with time, uncertainty, and incomplete set of assets. We consider two concepts of the sequential core for dynamic economies: the strong sequential core and the weak sequential core. Chapters 4 and 5 treat the strong and the weak sequential core, respectively, in the context of a two-period economy with assets. Chapter 6 considers the strong sequential core in the framework of a stationary exchange economy. Chapter 7 analyzes the strong sequential core in at stationary cooperative game.

To define the strong and the weak sequential core in a two-period economy with assets, we distinguish between the coalitional deviations that accur in period $t=0$ and in period $t=1$. The strong sequential core is a refinement of the classical core. It selects only those classical core allocations that are robust to coalitional deviations in period $t=1$, in the sense that there is a trade in assets such that the allocation at hand belongs to the core of the induced (static) subeconomy in each state. The central idea behind the concept of the weak sequential core is that of a credible deviation. A period $t=0$ deviation by a certain coalition is considered credible if it is robust to deviations by subcoalitions in period $t=1$. The weak sequential core consists of those allocations that are stable to deviations in period $t=1$ and at the same time are robust against all credible deviations in period $t=0$.

The formal problems addressed in Part II of the thesis are the following: (1) to assess the comparative statics of the strong and the weak sequential core with respect to the asset structure in the two-period economy, and (2) to investigate the non-emptiness properties of the strong and the weak sequential core.

The strong sequential core is weakly increasing in the number of assets. When the there is a strongly complete set of assets in the economy, the strong sequential core coincides with the classical core. Apart from the special case of a strongly complete asset structure, however, the non-emptiness of the strong sequential core in the two-period economy is difficult to guarantee. In particular, we show that in the finance economy the strong sequential core is generically empty, provided that the difference between the number of states and the number of assets is greater than one.

The weak sequential core may be empty even in the economy with no assets and three players all having identical state-independent elementary utility function. Furthermore, the comparative statics of the weak sequential core is complicated. Thus, we give an example of an economy where the weak sequential core is non-empty when no assets are present, and is empty when an appropriately specified asset is introduced into the economy.

Positive results on the non-emptiness of the strong sequential core are abtained in the framework of a stationary economy. We show that the strong sequential core of a stationary economy is non-empty, provided that the discount factor is sufficiently close to one. An analogous result can be obtained for a stationary cooperative game as well: the strong sequential core of a stationary cooperative game is non-empty, provided that (1) the instantaneous NTU-games in all states satisfy an additive $\pi$-balancedness condition, (2) at least one of these NTU-games satisfies a strong additive $\pi$-balancedness condition, and (3) the discount factor is sufficiently close to one.

The above findings indicate that neither the strong sequential core nor the weak se- 
quential core is an entirely coherent solution concept. Nevertheless we believe that the analysis undertaken in this thesis is valuable as an important step towards a general and a fully consistent concept of the sequential core. 



\section{Curriculum Vitae}

Predtetchinski Arkadi was born on June 9, 1979 in Novosibirsk, Russin. He graduated from high school in 1996. From 1996 till 2000 he studied Quantitative Economics at Novosibirsk State University. In Jume 2000 he was awarded Master's Degree in Economics with the specialty "Mathematical Models in Economics." From December 2000 till November 2004 Arkadi Predtetchinski was appointed as a PhD-student with the Faculty of Economics and Business Administration, Maastricht University. His research was supervised by Prof. dr. P. Jean-Jacques Herings and Prof. dr. Hans Peters.

Apart from subjects represented in this thesis, Arkadi Predtetchinski's research interests include general equilibrium theory, theory of incomplete markets, mathematical economics, and economics of uncertainty and information. 